Accepted for publication 8 May 2017.

Review article

\title{
Where are we? The anatomy of the murine cortical meninges revisited for intravital imaging, immunology, and clearance of waste from the brain.
}

\author{
Jonathan A. Coles $^{\mathrm{a},}$, Elmarie Myburgh ${ }^{\mathrm{b}}$, James M. Brewer ${ }^{\mathrm{a}}$, Paul G. McMenamin ${ }^{\mathrm{c}}$ \\ ${ }^{a}$ Centre for Immunobiology, Institute of Infection, Immunity and Inflammation, College of Medical, Veterinary and Life \\ Sciences, Sir Graeme Davis Building, University of Glasgow, Glasgow G12 8TA, United Kingdom. \\ ${ }^{b}$ Centre for Immunology and Infection Department of Biology, University of York, Wentworth Way, Heslington, York \\ YO10 5DD, United Kingdom. \\ ${ }^{c}$ Department of Anatomy \& Developmental Biology, \\ School of Biomedical and Psychological Sciences and Monash Biomedical Discovery Institute, Faculty of Medicine, \\ Nursing and Health Sciences, Monash University, 10 Chancellor's Walk, Clayton, Victoria, 3800, Australia. \\ *Corresponding author at: jonathan.coles@glasgow.ac.uk.
}

\begin{abstract}
Rapid progress is being made in understanding the roles of the cerebral meninges in the maintenance of normal brain function, in immune surveillance, and as a site of disease. Most basic research on the meninges and the neural brain is now done on mice, major attractions being the availability of reporter mice with fluorescent cells, and of a huge range of antibodies useful for immunocytochemistry and the characterization of isolated cells. In addition, two-photon microscopy through the unperforated calvaria allows intravital imaging of the undisturbed meninges with sub-micron resolution. The anatomy of the dorsal meninges of the mouse (and, indeed, of all mammals) differs considerably from that shown in many published diagrams: over cortical convexities, the outer layer, the dura, is usually thicker than the inner layer, the leptomeninx, and both layers are richly vascularized and innervated, and communicate with the lymphatic system. A membrane barrier separates them and, in disease, inflammation can be localized to one layer or the other, so experimentalists must be able to identify the compartment they are studying. Here, we present current knowledge of the functional anatomy of the meninges, particularly as it appears in intravital imaging, and review their role as a gateway between the brain, blood, and lymphatics, drawing on information that is scattered among works on different pathologies.
\end{abstract}

\section{Contents}

1. Introduction.

2. Experimental techniques.

2.1 Intravital imaging.

3. Overview of the fluid compartments within the cranium.

3.1. Blood.

3.2. Cerebrospinal fluid (CSF).

3.2.1. The extent of the cortical paravascular spaces.

3.2.2. Communication between the SAS and paravascular space.

3.3. Interstitial fluid (ISF).

3.4. Barriers separating blood, CSF and ISF.

3.4.1. The blood-brain barrier.

3.4.2. Blood -CSF barriers.

3.4.3. Other boundaries delimiting CSF space.

4. The anatomy of the cortical meninges of Murinae.

4.1. Ontogeny.

4.2. The dura.

4.2.1. Cell layers of the dura.

4.2.2. Vascularization of the dura.

4.2.3. Innervation of the dura.

4.2.3.1. Trigeminal innervation of the dura. 
4.2.3.2. Sympathetic innervation of the dura.

4.2.3.3. Parasympathetic innervation of the dura.

4.2.4. Lymph vessels in the cortical dura.

4.3. The leptomeninx

4.3.1. Innervation of the leptomeninx

5. Vascular dynamics in the meninges

5.1. Motor control of pial vessels.

5.2. Vascular dynamics in the dura.

5.2.1. Vasomotor control.

5.2.2. Extravasation of plasma proteins from dural vessels.

5.2.3. A note on headaches.

6. Extravascular transport of molecules within the cranium.

6.1. The 'macrocirculation' of CSF.

6.1.1. Secretion of CSF by the choroid plexuses.

6.1.2. Flow of CSF from ventricles to the surface of the cortex.

6.1.3. Efflux of CSF from the cranium.

6.2. Long-distance transport of solutes in the dorsal cortex.

6.2.1. Is there net flow of CSF along paravascular spaces?

6.2.2. A critique of the 'glymphatic' circuit.

6.3. Clearance of exogenous and endogenous molecules from the cortex.

6.4. Pathways for CSF in the meninges.

7. Where are we? Identifying locations in the meninges.

7.1. Meningeal landmarks for intravital imaging.

7.2. Mechanical separation of the dura, arachnoid and pia in rats and mice.

8. Immune cells in the meninges in health and disease.

8.1. Mast cells

8.2. Neutrophils

8.3. Microglia.

8.4. Perivascular immune cells.

8.5. Meningeal macorphages.

8.6. Dendritic cells.

8.7. T cells

8.8. Antigen presentation in the meninges.

8.9. Communication between the dura and CSF.

9. Conclusion.

\section{Introduction}

The prevalence of human meningitis (Christodoulides, 2013), the implication of the meninges in virtually all brain pathologies (Section 8), and the comparative accessibility of the meninges, naturally led to an early interest in their anatomy and function in man and large animal models (Willis, 1664). Surprisingly, even some major features of the anatomy are still disputed, and many unanswered questions on the physiology and immunobiology of the meninges and its consequences for the brain parenchyma remain subject to intense scrutiny. To address these questions, basic biomedical research now mainly uses rats and mice, exploiting the wide range of antibodies and fluorescent markers that are available and, in the case of mice, of genetically modified reporter strains in which particular cell types express fluorescent markers. Cells can be isolated from the meninges for cytometric analysis, or imaged on isolated meningeal tissue, or intravitally in the live mouse. Satisfactory interpretation of the data requires correct identification of the tissue compartments in which the observed cells are located, and knowledge of the anatomical and functional relations of these compartments with the rest of the animal. Interaction between tissue and flowing blood, which is fundamental to immunology and much parasitology, can only be studied in vivo, and in vivo imaging of the cerebral meninges is most conveniently done over the

Abbreviations:ASIC, Acid-sensing inward current channel; CCK, Cholecystokinin; CGRP, calcitonin gene; related peptide; CSF, Cerebrospinal fluid; CT, computer tomography; EAE, Experimental autoimmune encephalitis; ISF, Interstitial fluid; LCM, Lymphocytic choriomeningitis; LPS, Bacterial lipopolysaccharide; MCA, Middle cerebral artery; MCA, Middle cerebral artery occlusion; MHC, Major histocompatibilty complex; MMA, Middle meningeal 
artery; NPY, Neuropeptide Y; PACAP, Pituitary Adenylate Cyclase-Activating Polypeptide; PC-MRI, Phase-contrast magnetic resonance imaging; PVS, Perivascular space; RMCP, rat mast cell protease; SAS, subarachnoid space; SP, substance P; SSS, superior sagittal sinus; TRP, Transient receptor potential; VIP, Vasoactive intestinal polypeptide.

dorsal cortex rather than the basal aspect of the brain. Despite the importance of the meninges in brain research, most published drawings do not represent the reality of the murine cortical meninges, and information in the literature is dispersed because the publications on the various meningitides tend to be segregated by etiology. Hence, our intention here is to describe the anatomy of the meninges covering the dorsal cortex of mice, and to provide a guide to literature pertinent to their function and pathology. Although molecular differences between the meninges of mice and rats have been reported (Price and Flores, 2007) we draw on many findings in rats, and occasionally in other species, especially man.

The component layers of the cerebral meninges are attractively illustrated in a drawing published by Weed in 1923 (Fig.1A). Apposed to the cranium is the dense connective tissue of the outer, endosteal, layer of the dura mater. In a gross dissection, this outer layer appears to be fused to an inner layer (as in Fig. 1A) except where the two layers separate to house the dural venous sinuses or where the inner layer extends as a scythe-shaped structure, the falx cerebri, to fill the longitudinal fissure separating the hemispheres. The inner layer of the dura is apposed to the outer layer of the arachnoid. In the figure, the cells of the inner layers of the arachnoid are shown with downward extensions that cover bundles of collagen to form trabeculae. These traverse subarachnoid space (SAS) containing cerebrospinal fluid (CSF) then unite with the more delicate pia mater on the brain surface. The arachnoid layer, the SAS, and the pia are together known as the leptomeninx. As shown in Figs. 1A and B, the SAS appears to be continuous with a further CSF space surrounding arterioles that plunge into the brain (and venules that emerge from it). Flow of CSF, which can carry cytokines and cells of the immune system, supports communication between the meninges and the neural tissue within the parenchyma, and appears to be of great importance in maintaining the health of the brain (Section 6) or producing encephalitis (Section 8).

For researchers working on the dorsal meninges, particularly of rats and mice, the scheme of Figure 1A is misleading in two main ways. As Weed (1914a) himself wrote (for dog): "Over the convolutions the subarachnoid space is only of capillary thickness until it dips again into a fissure". Although this thinness may have been in part an experimental artifact, in mice, with their smooth cortex, the dorsal leptomeninx is indeed mainly thin, as indicated by 'lepto' (= 'thin, delicate'), and trabeculae are not apparent (Oda and Nakanishi, 1984; see Section 4.3). And, as in all species, the dura is a complex connective tissue encompassing a vascular network (Section 4.2.2), nerves (Section 4.2.3), and lymph vessels (Section 4.2.4), and containing numerous cell types. The dura is also known as the 'pachymeninx' where 'pachy' = 'thick'. In different pathologies, the immune response in the meninges may be predominantly in either the dura, or the arachnoid, or the pia. It is therefore important to be able to distinguish these compartments in dissected tissue and in intravital imaging (Section 7).

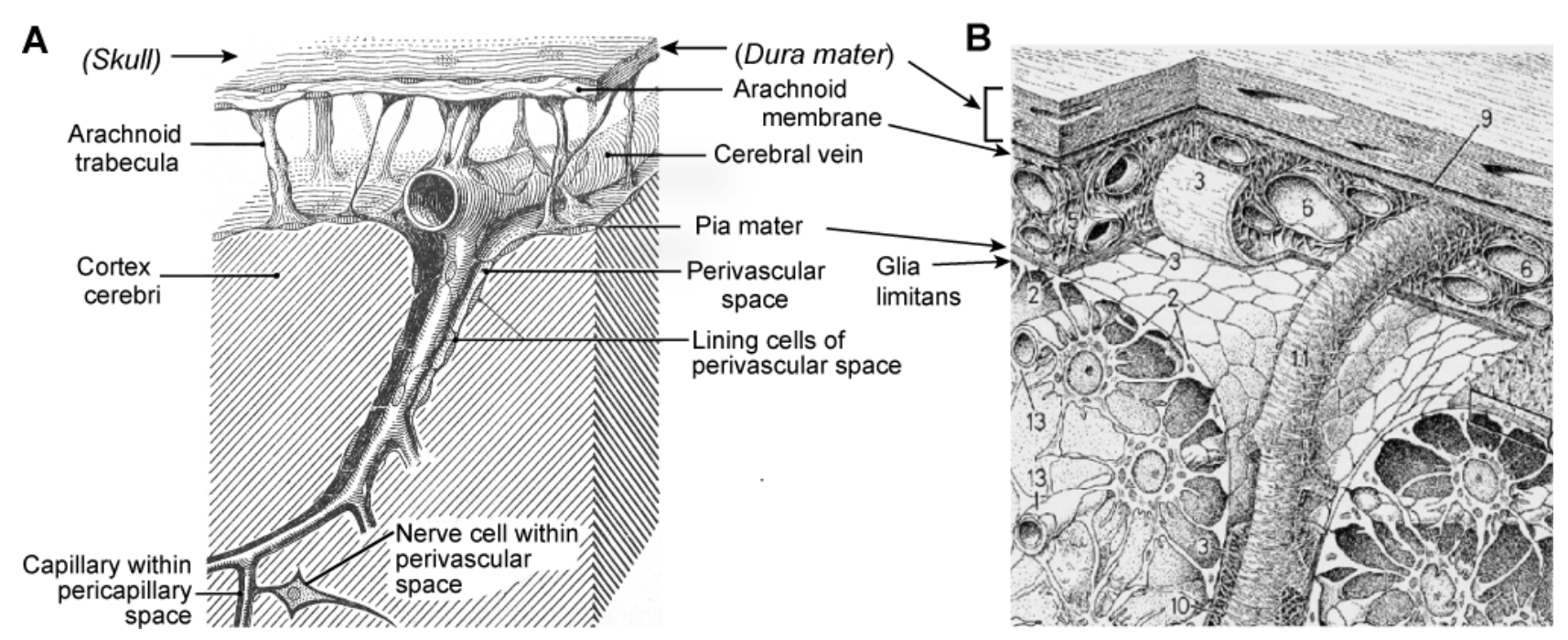


Fig. 1. Classical drawings of the cortical meninges. A. Scheme published by Weed (1923) for dog. In the original, the dura mater and skull are not labeled. In contrast to this drawing, the subarachnoid space over the dorsal cortex of Murinae is generally thin or occluded, while the dura is thick and complex. B. A scheme for rat by Krstić (1988), including results of electron microscopy studies. 1. Protoplasmic astrocyte. 2. Astrocyte endfeet forming the glia limitans. 3. Pia mater. 6. "Pial vessel". 9. Sub-dural space. 10. Perivascular space. 11. Penetrating blood vessel. 12. Capillary. This drawing is excellent, but underestimates the complexity of the dura and is unclear on paravascular space in the leptomeninx. Reproduced by permission of Springer Verlag.

\section{Experimental techniques}

Before histological techniques were developed to fix, embed, section, and stain tissues, neuroanatomists paid great attention to the meninges, which they could study in fresh tissue. Willis (1664) mentions dural (as well as pial) arteries, and knew that the dura could be the site of pain. Magendie (1825) showed that fluid flowed in the subarachnoid space, which was later delineated by injection of dye (Quincke, 1872; Key and Retzius, 1875). The introduction of lumbar punctures in humans by Quincke in 1891 (see Frederiks and Koehler, 1997), encouraged studies on the circulation of cerebrospinal fluid (CSF) and its route through the meninges (reviewed in Flexner, 1933 and Weed, 1938). Virtually all this work was naturally done on humans or other large mammals. Studies on smaller animals, notably the rat, became more common from the 1950s, with fluorescence microscopy being used by Rodriguez-Peralta, (1957) to characterize dural cells, and the subcellular resolution of electron microscopy being used to localize the barrier layer of the arachnoid (Nabeshima et al., 1975). More complete studies of rat meningeal anatomy and its morphogenesis were made by Andres (1967), Andres et al. (1987), Angelov and Vasilev (1989) and Fricke et al. (1997). Much interest in the meninges has been driven by the prevalence of migraine, since headache involves nerves with endings in the dura (Section 5.2.3). This has motivated the tracing of nerve trajectories between brain and meninges by using retrograde axonal transport of horseradish peroxidase (Steiger et al., 1982; O'Connor and van der Kooy, 1986) or dextran amines (Schueler et al., 2013), or by diffusion along the plasma membranes of fluorescent lipophilic dyes, such as DiI (Schueler et al., 2014; Section 4.2.3). Within the meninges themselves, fibers can be classified by labeling with antibodies against antigens, such as specific neuropeptides (Messlinger et al., 1993; Fricke et al., 1997). For pharmacological studies, a preparation consisting of a hemiskull with the dura attached allows collection of released compounds (Ebersberger et al., 1999a). Cells of the immune system are diversified into myriad subsets, so full characterization of a particular cell type requires analysis of its transcriptome or its proteome (Hume et al., 2013; Hume and Freeman, 2014) or, at least, identification of a panel of antigens (Becher et al., 2014). Cells have been isolated from mouse CSF (Carp et al., 1971), total meninges (Pfau et al., 1985), or a dural component alone (Morishima and Hayashi, 1978; Christy et al., 2013; Walker-Caulfield et al., 2015; Section 7.2), and also from rat spinal leptomeninx (Bartolomäus et al., 2009). The feasability of flow cytometric analysis of cell isolates from these relatively small volumes has been demonstrated (Christy et al., 2013). The location of immune cells within anatomical structures has been studied by immunocytochemistry on tissue sections (e.g.,Morishima and Hayashi, 1978; McMenamin, 1999; Reuter et al., 2001; McMenamin et al., 2003; Aspelund et al., 2015 and Louveau et al., 2015), or, better, on tissue wholemounts, e.g., Dimitriadou et al. (1997), or in vivo (Section 2.1).

2.1. Intravital imaging. Physiology and pathophysiology depend on movements of molecules and cells between compartments such as blood, tissue and lymph, and communication with the nervous system, so imaging in the living animal enables a wealth of information to be acquired more rapidly than from snapshots obtained from post-mortem tissue (Garside and Brewer, 2010; Ishii and Ishii, 2011). Intravital optical techniques lend themselves particularly to following the transport of marker substances and the motility of immune cells in smaller animals, such as the mouse (e.g., Bartolomäus et al., 2009; Kim et al., 2009; Pai et al., 2012; Louveau et al., 2015). Functioning blood vessels in the cortical meninges have long been observed through cranial windows (see Forbes, 1928), and photographed since Jacobi and Magnus (1925). However, excision of calvarial bone in mouse may inadvertently remove part of the dura (Syed et al., 2012 and Walker-Caulfield 
et al., 2015), also causes degranulation of meningeal mast cells (Markowitz et al., 1989 and Levy et al., 2007), and has been shown to perturb function in the pia and gray matter (Olesen, 1987 and Xu et al., 2007). Imaging through unperforated skull bone after mechanically thinning to transparency reduces these problems (Williamson et al., 1997a; Xu et al., 2007), although trauma to trigeminal nerve terminals in the calvaria (Kosaras et al., 2009) may theoretically cause inflammation of the dura by the axon reflex (see Section 4.2.3). Classical 'one-photon' microscopy then gives sufficient resolution to measure changes in the diameters of pial arteries (Williamson et al., 1997a; Akerman et al., 2002c), and further information about blood flow can be obtained by laser Doppler flowmetry (Holloway and Watkins, 1977; Eyre et al., 1988), laser speckle contrast imaging (Fercher and Briers, 1981; Kazmi et al., 2015) or modifications of optical coherence tomography (Li et al., 2014). Greater spatial resolution of cells and subcellular details in the intact, undisturbed, meninges can be obtained with two-photon microscopy (Kim et al., 2009; Drew et al., 2010; Yang et al., 2010; Fumagalli et al., 2011; Iliff et al. 2012; Shih et al., 2012; Coles et al., 2015). Time resolution is adequate for 3D imaging of movements of immune system cells; erythrocyte velocity can be measured by line-scanning (Kleinfeld et al., 1998). For imaging at depth in the cortex, only slight loss of resolution has been reported for the thinned skull preparation compared to removal of the bone (Isshiki and Okabe, 2014). Although two-photon microscopy of the mouse was initially developed to study neurons in the gray matter (Svoboda et al., 1997; Grutzendler et al., 2002), it has become apparent since the work of Kim and Dustin (2006) that it is ideally suited to imaging the many pathological processes that occur in the cortical meninges (Pai et al., 2012). Relating the images to the anatomy has posed some problems that it is the intention of this review to address.

The basis of conventional fluorescence microscopy is that a fluorescent molecule can be excited by absorbing a high-energy photon (corresponding to a short wavelength) and it then emits a lower energy photon (corresponding to a longer wavelength). In two-photon microscopy, the molecule is bombarded so intensely with lower energy photons that occasionally two arrive simultaneously and their combined energy is sufficient for excitation (Göppert-Mayer, 1931). The molecule then emits a photon of the usual wavelength. Continuous very intense illumination would overheat the tissue, and to avoid this, the excitation beam is composed of brief (femtosecond) pulses and is focused at only one point which then scans the focal plane. In this way, the peak flux of photons at the focal point can be very high, while the average power absorbed by the tissue is low. Practically, this means using the same type of scan head as in a conventional confocal microscope, but with a 'femtosecond' near-infrared laser as a source. For imaging biological tissues, two-photon microscopy has several advantages (Denk and Svoboda, 1997; Helmchen and Denk, 2005). A major one is that the near-infrared excitation beam can penetrate deeper into tissue than visible light, while causing less tissue damage, or bleaching of fluorophores (Helmchen and Denk, 2005). Fluorescence (in the visible) is produced only at the focal point of the excitation beam so, even though the fluorescence light is scattered as it passes back through the tissue, it all comes from one point in the tissue and can contribute to forming an image as the object plane is scanned. Light collection in this way is more efficient than in a 'one photon' confocal microscope, in which a pinhole selects only a small part of the fluorescent light (Denk et al., 1990). The maximum depth of imaging depends strongly on the time that can be spent on scanning each XY plane (so that enough photons can be collected), and on the spatial resolution desired (so that there are enough photons per pixel). At one extreme, large blood vessels containing a long-wavelength fluorescent dye can be imaged to a depth of several hundred microns (Theer and Denk, 2006)). At the other extreme, trypanosomes, the protozoans responsible for sleeping sickness, which can move tens of microns per second, are difficult to image clearly at depths more than about 100 microns below the skull. Perhaps surprisingly, the cortical meninges of mouse, and their underlying neural brain, form a structure that is relatively simple to image in vivo. The skull provides a conveniently rigid support for the soft tissue (working drawings are given in Coles et al., 2017) and thinning the skull requires only some patience rather than skill. With more careful surgery, a glass window allowing repeated imaging can be inserted (Drew et al., 2010; Marker et al., 2010; Yang et al., 2010; Shih et al., 2012). 
Table 1 lists some of the fluorescent dyes and genetically modified proteins that have been used for intravital imaging. In addition to fluorescence, two-photon excitation can produce a signal from some non-fluorescent materials by second harmonic generation (SHG, see Campagnola and Loew, 2003). A component of the scattered light has exactly half the wavelength of the exciting light and, for practical purposes, can be treated like fluorescence and used to construct images. Collagen and skull bone both show strong SHG and their images provide helpful landmarks for locating other objects in the meninges (Mohler et al., 2003 and Coles et al., 2015).

Table 1. Fluorescent labels for transcranial intravital imaging

\begin{tabular}{|c|c|c|c|c|c|}
\hline Route & Dye & $\begin{array}{l}\text { Structures } \\
\text { Labelled }\end{array}$ & $\begin{array}{l}\text { Emission peak } \\
\text { (approximate) }\end{array}$ & References & Comments \\
\hline \multirow[t]{9}{*}{ Intravenous } & $\begin{array}{l}\text { RhodamineB } \\
\text { dextran } 70 \mathrm{kD}\end{array}$ & Blood plasma & $590 \mathrm{~nm}$ & {$[1,2]$} & \\
\hline & $\begin{array}{l}\text { Fluorescein } \\
\text { dextran } 10 \text { or } 70 \\
k D\end{array}$ & Blood plasma & $520 \mathrm{~nm}$ & $\begin{array}{l}{[3]} \\
{[2]}\end{array}$ & \\
\hline & $\begin{array}{l}\text { Texas Red dextran } \\
3 \mathrm{kD} \text { or } 70 \mathrm{kD}\end{array}$ & Blood plasma & $605 \mathrm{~nm}$ & [4] & \\
\hline & $\begin{array}{l}\text { Cascade Blue } \\
\text { dextran } 10 \mathrm{kD}\end{array}$ & Blood plasma & $420 \mathrm{~nm}$ & [4] & \\
\hline & Quantum dots & $\begin{array}{l}\text { Blood plasma, } \\
\text { extravasation, } \\
\text { phagocytes }\end{array}$ & Various & {$[5,6,7,8]$} & $\begin{array}{l}\text { Q Tracker } \\
\text { (Invitrogen) }\end{array}$ \\
\hline & $\begin{array}{l}\text { Sulphorhodamine } \\
101\end{array}$ & Astrocytes & $592 \mathrm{~nm}$ & [9] & \\
\hline & Aminoacridine & $\begin{array}{l}\text { Nuclei of dural } \\
\text { cells }\end{array}$ & & [10] & \\
\hline & Furamidine & $\begin{array}{l}\text { Nuclei of } \\
\text { vascular } \\
\text { endothelial cells } \\
\text { and dural cells }\end{array}$ & $464 \mathrm{~nm}$ & {$[7,11,12]$} & $\begin{array}{l}\text { Related } \\
\text { compounds [13] } \\
\text { show similar } \\
\text { behavior }\end{array}$ \\
\hline & Evans Blue & $\begin{array}{l}\text { Blood, } \\
\text { extravasation }\end{array}$ & $700 \mathrm{~nm}$ & {$[8,14]$} & \\
\hline $\begin{array}{l}\text { Superfusion of } \\
\text { skull }\end{array}$ & Sulforhodamine B & Osteoclasts & $592 \mathrm{~nm}$ & $\begin{array}{l}\text { [9] This paper, } \\
\text { Fig. } 7 \mathrm{G}\end{array}$ & \\
\hline \multirow[t]{2}{*}{$\begin{array}{l}\text { Infusion into } \\
\text { the cisterna } \\
\text { magna }\end{array}$} & $\begin{array}{l}\text { Texas Red } 2-70 \\
\text { kD }\end{array}$ & $\begin{array}{l}\text { Perivascular } \\
\text { space, } \\
\text { subarachnoid } \\
\text { space }\end{array}$ & $605 \mathrm{~nm}$ & {$[4,7]$} & \\
\hline & Sulforhodamine B & $\begin{array}{l}\text { paravascular } \\
\text { subarachnoid } \\
\text { space }\end{array}$ & $592 \mathrm{~nm}$ & $\begin{array}{l}\text { This paper, Fig. } \\
3 \mathrm{C}\end{array}$ & \\
\hline \multicolumn{6}{|l|}{ GM promoter } \\
\hline CD2 & GFP & T lymphocytes & $488 \mathrm{~nm}$ & {$[1,15,16]$} & \\
\hline CD2 & DsRed & T lymphocytes & $586 \mathrm{~nm}$ & {$[7,15][16]$} & \\
\hline CD11c & EYFP & $\begin{array}{l}\text { Dendritic cells, } \\
\text { some } \\
\text { macrophages }\end{array}$ & $527 \mathrm{~nm}$ & [17] & \\
\hline $\mathrm{CX}_{3} \mathrm{CR} 1$ & GFP & $\begin{array}{l}\text { microglia, } \\
\text { macrophages }\end{array}$ & $488 \mathrm{~nm}$ & $\begin{array}{l}{[8,18] \text { This }} \\
\text { paper, Fig. 7DE }\end{array}$ & \\
\hline
\end{tabular}




\begin{tabular}{|l|l|l|l|l|l|}
\hline Tie2 & GFP & $\begin{array}{l}\text { Vascular } \\
\text { endothelium }\end{array}$ & $488 \mathrm{~nm}$ & {$[4,19]$} & \\
\hline NG2 & DsRed & $\begin{array}{l}\text { Arteries } \\
\text { (pericytes, } \\
\text { vascular smooth } \\
\text { muscle) + some } \\
\text { glial cells }\end{array}$ & $586 \mathrm{~nm}$ & {$[3,4]$} & \\
\hline
\end{tabular}

1. Fumagalli et al. (2011). 2. Verant et al. (2007). 3. Zhu et al. (2008). 4. Iliff et al. (2012). 5. Kim et al. (2009). 6. Roth et al. (2014). 7. Coles et al. (2015). 8. Shaw et al. (2015). 9. Appaix et al. (2012). 10. Rodriguez-Peralta (1957). 11. Myburgh et al. (2013). 12. Mathis et al. (2006). 13. Mathis (2007). 14. Saria (1983). 15. Veiga-Fernandes et al. (2007). 16. Drobizhev et al. (2011). 17. Lindquist et al. (2004). 18.Jung et al. (2000). 19. Motoike et al. (2000). Ehrlich (1877) and Goldmann E. E. (1909) made extensive studies of intravital staining of cells and tissues, using fluorescent dyes that may be useful for two-photon imaging.

\section{Overview of the fluid compartments within the cranium.}

To a first approximation, extracellular fluids within the cranium can be categorized as blood, CSF, interstitial fluid of the parenchyma (ISF), extracellular fluid of the dura, and lymph. There are sharp boundaries and clear differences in composition between blood, CSF, and the extracellular fluid of the dura, but some results suggest that CSF, ISF and lymph flow one into another in a continuous pathway (Section 6).

3.1. Blood. The gross vascular anatomy of the mouse brain has been reconstructed from data obtained by X-ray computer tomography (CT; Ghanavati et al., 2014) and by CT combined with MRI (Dorr et al., 2007). The mouse cerebrum, like that of humans, is supplied mainly by the paired internal carotid arteries. These, together with an input from the vertebral artery, anastomose at the base of the brain to form the circle of Willis from which arteries branch laterally and then dorsally over the cortex, until arterioles (Dahl, 1973) plunge abruptly downwards into the parenchyma (Figs $1 \mathrm{~A}, \mathrm{~B}, 2 \mathrm{~A}, \mathrm{H})$. The trajectories of the superficial vessels vary greatly among individual mice - Fig. 2A shows an average -, and, more generally, the architecture of the parenchymal vascularization has no clear association with the columnar arrangement of neuronal function (Blinder et al., 2013). However, the area of the cerebral cortex most conveniently accessible to craniotomy or transcranial imaging (over the parietal cortex) is usually supplied medially by the anterior cerebral artery and more laterally by the middle cerebral artery (MCA) (Dorr et al., 2007; Fumagalli et al., 2011). The feeding arteries and draining veins lie horizontally above the cortical surface or embedded in it (Fig. $2 \mathrm{H}$ ), the veins generally coursing medially to drain to venous sinuses in the dura, especially the superior sagittal sinus (Fig. 2A). The dura and calvaria do not receive blood from the cerebral arteries; instead, as described in Section 4.2.2, they are supplied from the external carotid systems via the meningeal arteries (well-illustrated in Vieussens, 1684). 

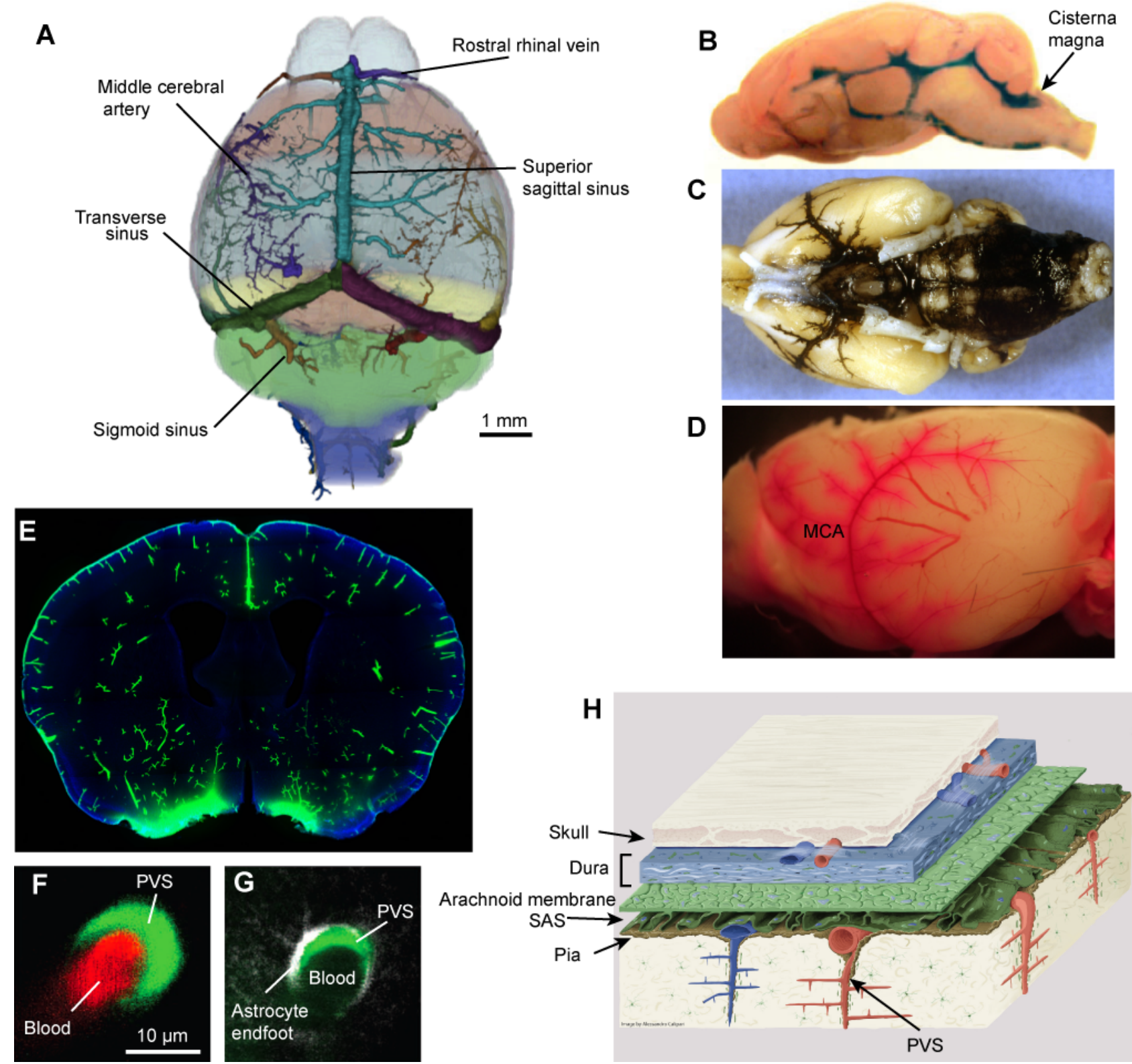

Figure 2. Overview of fluid compartments. A. Gross anatomy of surface vessels of the mouse brain. Of the dural vessels, the venous sinuses are shown, but the middle meningeal artery has been removed digitally. The 3D vascular architecture was obtained by a combination of X-ray computed tomography and NMR imaging on four mice and the results averaged: there is great variation between individuals. From Dorr et al., 2007, reproduced with permission from Elsevier. B. Labeling of internal CSF spaces on a parasagittal section of mouse brain after infusion of methylene blue in the lateral ventricles Modified from Planaguma et al., 2015. C. The ventral aspect of a rat brain after injection of Indian ink into the cisterna magna. The subarachnoid space of the circle of Willis and the middle cerebral arteries are labeled. (By courtesy of S. Kida and R.O. Weller). D. Sulforhodamine (M.Wt 599, orange) injected in the cisterna magna of a mouse labels wide spaces adjacent to the middle cerebral artery (MCA). E. Thirty min after injection of fluorescence labeled ovalbumin (green) in the cisterna magna of mouse, leptomeningeal space, paravascular spaces of penetrating vessels, and interstitial space in ventral brain are labeled. From Iliff et al., 2013b. F,G. Two-photon microscopy of penetrating arteries in mouse cortex with perivascular space labeled by infusion in the cisterna magna of $2000 \mathrm{kD}$ dextran conjugated with a fluorophore (shown as green). In (F), blood is labeled with Texas Red dextran and (G) is from a reporter mouse expressing GFP under control of the GFAP promoter (shown as white). Modified from Iliff et al., 2012. H. Schematic drawing of the rodent cortical meninges showing the arachnoid membrane (green) separating the leptomeninx from the dura, which contains many layers of cells and blood vessels.

The volume of blood in the rat brain has been measured by synchrotron radiation computed tomography (Adam et al., 2005) giving values 1.65 to $4.1 \mathrm{~mL} / 100 \mathrm{~g}$, and by in vivo MRI, giving values of 4-5\% in the cortex (Barbier et al., 2001; Coquery et al., 2014). In mouse, two-photon imaging of the cortex has given a volume fraction of $2-2.4 \%$ (Verant et al., 2007). 


\subsection{Cerebrospinal Fluid.}

Spaces filled by cerebrospinal fluid (CSF) can be visualized by injecting a marker dye in the lateral ventricles (Locke and Naffziger, 1924), or the cisterna cerebello-medullaris (or 'cisterna magna'), which lies beneath the atlanto-occipital membrane posterior to the cerebellum (Fig. 2B; Key and Retzius, 1875). From the cisterna magna, CSF flows into the capacious arachnoid cisternae of the ventral brain and circle of Willis (Fig. 2C), and into channels beside the cerebral arteries, from where it can spread through the cortical leptomeninges (Fig. 2D), but not the dura (Section 4.3). This cranial CSF space communicates with the spinal meninges (Magendie, 1825; Key and Retzius, 1875). CSF, sampled from the cisterna magna, has a tightly-regulated ionic composition, significantly different from that of blood, and contains only low concentrations of glucose, and of amino acids except glutamate (Mott, 1910; Bito et al., 1966; Davson, 1976; Husted and Reed, 1976, 1977). However, the composition of CSF is not uniform throughout the CSF space (Bito and Davson, 1966), so there are concentration gradients which could only be maintained by exchange of material with tissue or with the other fluid compartments (blood, ISF or lymph), as discussed in Section 6.

A CSF space also extends along the vessels that penetrate into the brain from the meninges. It has long been suggested that lymph-like fluid flows along this perivascular space, much as in lymph vessels outside the brain (His, 1865, see Section 6.4), and, more recently, and controversially, that the flow may be important for the elimination of amyloid $\beta$ and avoidance of neurodegenerative diseases (see Section 6.5). Two basic questions that have given rise to conflicting answers are: how far does this perivascular CSF space accompany the arborization of blood vessels in the parenchyma? And is the perivascular space continuous with the SAS?

3.2.1. The extent of perivascular space in the cerebral cortex. The endothelium of a penetrating arteriole is surrounded by smooth muscle cells covered by a basement membrane. Outside this vascular tube is a perivascular space, then a cellular layer continuous with the pial membrane, and a parenchymal basement membrane covering a layer of astrocyte endfeet continuous with the glia limitans of the pia (Figs. 1B, 2H). According to Woollam and Millen (1955), perivascular space was first described by Pestalozzi (1849), but it has often been called 'Virchow-Robin' space in acknowledgement of early descriptions by Virchow (1851) and Robin (1859). However, Virchow described local, macroscopic, and usually pathological, perivascular spaces, and this restricted sense is used in current literature on MRI (Groeschel et al., 2006; Lim et al., 2015). Robin studied dissected vessels and shows continuous spaces wider than has been found in subsequent work. For these reasons, the term 'Virchow-Robin space' might usefully be reserved for pathologists, or for the perivascular space close to the pial surface (Tarasoff-Conway et al., 2015).

Electron microscopists generally observe a patent perivascular space outside penetrating arterioles (e.g., Rangroo-Thrane et al., 2013) but not terminal arterioles, capillaries or venules (Maynard et al., 1957; Nelson et al., 1961; Mathiisen et al., 2010), although Frederickson and Low (1969) found that occlusion of the space round capillaries was true only in "most areas of the brain". Jones (1970) found that in rat cortex, the space was usually present as deep as the upper part of neuronal layer III (about $400 \mu \mathrm{m}$ ) but could extend as far as neuronal layer VI (1.5 mm) along the larger vessels, before being occluded. Workers studying transfer of molecules from blood to brain tissue support the close apposition of astrocyte endfeet to the vascular tube (Lehre et al., 1995), and Gordon et al. (2007) conclude from immunohistochemistry on fixed tissue that "all cerebral arterioles ... are encased by astrocyte endfeet". Close contact is also implied by the wellestablished control of arteriolar and capillary diameter by signals from astrocyte endfeet (Peppiatt et al., 2006; Iadecola and Nedergaard, 2007; Stefanovic et al., 2008; Attwell et al., 2010). In contrast, as discussed in Section 6.4, much work shows that CSF flows through spaces surrounding arterioles (and venules) and that tracers in CSF can enter a pericapillary space. There may be regional differences (Frederickson and Low, 1969) or perhaps the difference is a matter of terminology and technique, as in vivo imaging of infused dye does not by itself distinguish between 'space' and 'basement membrane', and, in fixed tissue, extracellular spaces tend to be occluded, and marker molecules are bound to host tissue. Concerning the larger penetrating arterioles, the vascular tube 
appears not to be centrally placed, but is in contact with one part of the sheath (Fig. 2F,G), so that both paravascular flow and astrovascular signaling should be possible.

3.2.2. Communication between the $S A S$ and paravascular space. Electron microscopical studies of blood vessels entering the cortex have suggested that paravascular space is not continuous with SAS. Before plunging into the brain, some, perhaps all, surface arterioles become embedded in a depression in the brain surface where they lie on the basement membrane and astrocyte layer of the glia limitans, and are separated from the SAS by pial cells (Jones, 1970; Krisch et al., 1984; Ichimura et al., 1991). The outer boundary of the perivascular space is then a continuation of the glia limitans (Ichimura et al., 1991). Alternatively, the arteriole dives directly from the SAS, and for a short distance takes with it a cell covering that is reflected to join the pia mater (Krahn, 1982; Weller, 2013). In both cases, a layer of pia mater is interposed between the SAS and the deeper perivascular space, so that, according to Krisch et al. (1984): "The perivascular compartment is not in communication with the arachnoid space". In apparent contradiction to this, repeated experiments have found that marker molecules introduced into CSF can pass readily into paravascular space (Section 6.2). This was shown for exogenous proteins in cat by Klatzo et al. (1964) and Rennels et al. (1985) and in rat by Wagner et al. (1974) and for proteins and $2000 \mathrm{kDa}$ dextran in mice by Iliff et al. (2012, 2013b) (Fig. 2E,F,G). Ichimura et al. (1991) reported only slow movement of Indian ink from SAS into paravascular space, but in their preparation the dura had been removed, and others have also found that the movement was greatly slowed when the dura was pierced (Iliff et al. 2012; Rangroo-Thrane et al., 2013). In disease, immune cells can pass from the meninges into perivascular space of penetrating arterioles (Ransohoff et al., 2003). This is true of monocytes in mouse after brain ischemia (Miro-Mur et al., 2015; Sections 7.3,7.8), and of T cells, which (in rat spinal cord), apparently originate in the inflamed leptomeninx and are clearly seen descending perivascular spaces (Bartolomäus et al., 2009). In mouse cortex, in advanced trypanosomiasis, occasional $\mathrm{T}$ cells have been seen below the level of the pia (Coles et al., 2015). In humans, movement of MRI contrast agent from SAS to presumed perivascular space has been reported (Saylisoy et al., 2014). Some electron micrographs have suggested that the pia mater is incomplete (Jones, 1970) or fenestrated (Krahn, 1982). Taken together, the literature concurs that a layer of pial cells is indeed interposed between the perivascular space and the SAS, but that it offers little resistance to the passage of molecules as large as $2000 \mathrm{kDa}$, or the passage of cells.

3.3. Interstitial fluid. The interstitial fluid that bathes neurons and glia of the parenchyma is the medium through which essential molecules (such as glucose) are delivered and unwanted ones (such as excess amyloid $\beta$ ) are removed. It is well established that the fraction $a$ of the parenchyma occupied by interstitial fluid is about $20 \%$ in anesthetized mammals (Nicholson and Phillips, 1981; Hrabetova and Nicholson, 2007). Neuronal activity causes $a$ to fall (Dietzel et al., 1980) and it has recently been reported that while $a$ is about $23 \%$ in the cortex of sleeping or anesthetized mice, it is only $14 \%$ in awake mice (Xie et al., 2013). Apart from the plasma membranes of the cells of the cortical parenchyma (neurons, astrocytes, microglia) interstitial fluid is bounded by the blood-brain barrier surrounding capillaries (Section 3.4), and by the layer of astrocyte endfeet that face the perivascular spaces of arterioles and venules (Mathiisen et al., 2010) and also form the glia limitans beneath the pia mater (Fig.1B). At the surfaces of the ventricles, the ISF is separated from CSF by ependymal cells and tanycytes (Feng et al., 2011).

Diffusion, even of large protein molecules, along microscopic extracellular clefts is limited neither by their narrowness (34 - $68 \mathrm{~nm}$ wide in rat cortex (Thorne and Nicholson, 2006), nor by their tortuosity (Nicholson et al., 1979; Hrabetova and Nicholson, 2007). However, the macroscopic extracellular diffusion of molecules through the tissue is severely limited because the movement is confined to the extracellular volume fraction, because the molecules must follow a tortuous path, and because the efficacy of transport by diffusion falls off as the square of distance (Nicholson et al., 2000). Hence the composition of ISF is strongly affected by local transmembrane transport and varies from place to place and in time. Many of the available probes for measuring solute concentrations are large relative to the width of the clefts (e.g., a diameter $>0.2 \mathrm{~mm}$ for 
microdialysis probes). Nevertheless the artifactual space they create contains fluid in approximate equilibrium with a network of clefts, and a measurement averaged in space and time can be made (Bito et al., 1966). In this way, changes in the concentration of glucose, lactate and other metabolites have been measured in response to neural activity (Caesar et al., 2008). Ion-selective microelectrodes can be much smaller (down to $<1 \mu \mathrm{m}$ ) and have shown different values of baseline $\left[\mathrm{K}^{+}\right]$in different parts of rat brain, with the highest value, $3.35 \mathrm{mM}$, being in cortex (Moghaddam and Adams, 1987). In these experiments, the dura had been removed, so it is possible that the trauma caused interstitial $\left[\mathrm{K}^{+}\right]$to increase, and the lower values $(\approx 2.5 . \mathrm{mM})$ recorded further from the surface are more physiological. Neuronal activity causes rapid changes in $\mathrm{K}^{+}, \mathrm{Ca}^{2+}$ and $\mathrm{Na}^{+}$ (Heinemann and Lux, 1975; Dietzel et al., 1980; Coles and Poulain, 1991), that have been measured on time scales down to seconds (Ransom et al., 1987). Reported values for glucose concentration, measured either by biosensor or microdialysis, are about $0.35 \mathrm{mM}$ in the striatum of awake rats and 1.5 - $3.3 \mathrm{mM}$ in various brain regions of anesthetized rats (see Lowry et al., 1998). The development of genetically encoded extracellular optical sensors should lead to better temporal and spatial resolution (Liang et al., 2015).

The fluid that fills the extracellular spaces of the dura is well isolated from the CSF of the leptomeninx and its composition is unknown (Penfield, 1923; Bennett, 1969; Cserr and Bundgaard, 1984; Haines, 1991).

\subsection{Barriers separating blood, CSF and ISF.}

3.4.1. The blood-brain barrier. It was known by 1900 that strychnine and other neuroactive chemicals produce behavioral effects if they reached the brain, but have no such effects when injected in blood (Biedl and Kraus, 1898; Lewandowsky, 1900), suggesting the presence of a 'blood-brain barrier' (BBB; Stern, 1923a,b). Similarly, dyes such as trypan blue (Goldmann, 1913; Spatz, 1934; Broman, 1940) or aminoacridines (Rodriguez-Peralta, 1957), when injected in the blood, leak from capillaries and stain tissue components throughout the body, but leave unstained most parts of the brain parenchyma. Although experimental opening of the blood-ISF barrier can cause seizures (Fieschi et al., 1980), the barrier cannot be hermetic since, to maintain brain metabolism and homeostasis, a large range of ions and molecules must be transported across it (Walter, 1930; Oldendorf, 1971; Davson, 1976).

The tightness of the BBB depends on metabolically sustained transmembrane ion gradients (De Bock, 2013) and therefore fails in ischemia. Leakage of dye through the BBB has been demonstrated in some encephalitides (Mathur et al., 1992; Kim et al., 2009; Walker-Caulfield et al., 2015), including cerebral malaria, in which arrest of parasitized erythrocytes on the brain capillary endothelium is associated with leaks in the BBB (Nacer et al., 2014). But dye leakage is not necessarily correlated with parenchymal infiltration by blood monocytes (Menasria et al., 2015) or with clinical symptoms in relapsing EAE (Walker-Caulfield et al., 2015). It has also been suggested that the BBB is broached by transmigration of activated T lymphocytes, but the only support for this comes from cultures of brain microvessel endothelium (Graesser et al., 2002). In contrast, there is good evidence that $\mathrm{T}$ cells enter CSF space from the choroid plexus and leptomeninges, and can then enter the parenchyma (Ransohoff et al., 2003; Bartolomäus et al., 2009; Reboldi et al., 2009).

For an intravenous drug to act on the brain it must pass through the $\mathrm{BBB}$, and, mainly for that reason, the BBB is the subject of intense study. The results underline its complexity and are frequently reviewed (Brightman, 1977; Abbott et al., 2010; De Bock et al., 2013; Ben-Zvi et al., 2014; Keaney and Campbell, 2015). We confine ourselves to a brief consideration of its anatomy.

Electron microscopy and immunofluorescence microscopy show that the capillary endothelium is covered by a basal lamina which in turn is about one third covered by pericytes. Where there are no pericytes, the basal lamina is covered by astrocyte endfeet, which also partly cover the pericytes (Mathiisen et al., 2010). Historically, it had been suggested that the layer of astrocyte endfeet, the 'glia limitans' might be the site of the blood brain barrier (Gärtner, 1927). However, further investigation showed that the barrier to the entry of foreign molecules is the endothelium of the vessel wall (see Broman, 1949), and this conclusion was supported by electron 
microscopic studies showing that vascular endothelial cells in the brain are connected by tight junctions impermeable to horseradish peroxidase (Reese and Karnovsky, 1967; Brightman and Reese, 1969). In contrast, there are gaps between the astrocyte endfeet (Reese and Karnovsky, 1967). Transfer across the capillary endothelium involves transport across the intra- and abluminal membranes, which are equipped with specific transporters (Kubo et al., 2015), notably for glucose (Pardridge et al., 1990; Kacem et al., 1998). Other transporters, many of them members of the ATPbinding cassette superfamily, have the opposite function of hindering transendothelial transport, by exporting foreign molecules back into the capillary lumen (Loscher and Potschka, 2005). Unlike extracranial capillaries, brain capillaries normally show little vesicular transfer (Brightman, 1977; Ben-Zvi et al., 2014).

It is expected from the gaps between them (Reese and Karnovsky, 1967), that the astrocyte endfeet may hinder, but not prevent, passage of foreign tracer molecules (Mathiisen et al., 2010), as is the case for the morphologically similar layer of astrocyte endfeet at the cortical surface and surrounding the perivascular space of brain arterioles (Section 3.4.3; Krisch et al., 1983, 1984; Iliff et al., 2012;Smith et al., 2016). The endfeet membranes contain many protein molecules, often in arrays (Landis and Reese, 1981), the potassium channel, Kir4.1, and the water and gas channel, aquaporin 4, being predominant (Nagelhus et al., 2004). The movement of potassium ions and water are important for cell volume regulation, and the gas conducting properties of aquaporin may be important for the delivery of oxygen (Thrane et al., 2013), as, although oxygen passes readily through a lipid membrane (Fischkoff and Vanderkooi, 1975), it is impeded by proteins (Boron, 2010). Glucose transporters, as expected, are found in astrocyte endfeet apposed to capillaries, rather than lining periarteriolar space (Kacem et al., 1998).

Astrocytes also participate in the development and maintenance of a 'neurovascular unit' in which local angiogenesis matches the metabolic requirements of the neurons and astrocytes (Jiang et al., 1995; Hawkins and Davis, 2005; Iadecola and Nedergaard, 2007), and signals from astrocytes induce tightness of the capillary endothelium (Janzer and Raff, 1987).

3.4.2. The blood-CSF barrier. Apart from the choroid plexuses, which secrete CSF into the ventricles, blood is separated from CSF by the walls of surface arteries and veins in the leptomeninx, and by the walls of penetrating arterioles and venules surrounded by perivascular CSF space in the brain itself. The endothelial cells of these vessels, like those of brain capillaries, are connected by tight junctions, exclude horseradish peroxidase and constitute a relatively impermeable barrier (Reese and Karnovsky, 1967). In the meninges, CSF is bounded externally by the arachnoid barrier layer, which separates it from the extracellular fluid of the dura (Section 4.3). Since the blood vessels of the dura are relatively permeable, the arachnoid barrier layer can be considered part of the blood-CSF barrier (Yasuda et al., 2013). The efficacy of the blood-CSF barrier is illustrated by that fact that a tracer molecule, such as thiourea, injected intravenously, reaches equilibrium more rapidly in brain tissue than in CSF (Davson, 1976), although much of this difference will be because the total surface area of the capillaries greatly exceeds the surface area of the larger vessels, rather than because the specific resistances of the vessel walls are different.

3.4.3. Other boundaries delimiting CSF space. Small dye molecules (Iliff et al., 2012) and foreign proteins (Rennels et al., 1985) pass only slowly from CSF in the ventricles across the ventricular ependyma and into the ISF. In contrast, neuroactive compounds (Biedl and Kraus, 1898; Lewandowsky, 1900), and small (3 kD) dye molecules (Iliff et al., 2012) appear to move with little restriction from subarachnoid and peri-arteriolar space to ISF.

\section{The anatomy of the cortical meninges of Murinae}

\subsection{Ontogeny.}

The embryonic meninges participate in the development of the brain, for example, by releasing retinoic acid, which stimulates neurogenesis (Siegenthaler et al., 2009; Siegenthaler and Pleasure, 2011). It had been speculated that the dura and leptomeninx had separate embryonic origins, the leptomeninx arising from the neural crest and the dura from mesoderm. However, in quail-chick chimeras it was found that all layers of the meninges covering the forebrain develop from the neural crest, while all layers covering the rest of the brain and the spinal cord derive from mesoderm 
(Couly and Le Douarin, 1987; Siegenthaler and Pleasure, 2011). In Murinae, too, there is evidence for a common origin of dura and leptomeninx. In mouse (Zajicova, 1987) and rat (Angelov and Vasilev, 1989) the dura appears to develop from a primitive, undifferentiated, meninx, and in mice with the congenital hydrocephalus mutation (Kume et al., 1998), or with targeted disruption of the transcription factor Foxc1 (Siegenthaler et al., 2009), both the dura and the leptomeninx are absent.

\subsection{The dural layer.}

Historically, dissection of humans and other large mammals led to the description of the dura mater, or pachymeninx, as a sheet of tough, collagenous, connective tissue, which detaches from the calvaria and can be mechanically removed from the underlying leptomeninges. The dura is well vascularized by the meningeal arteries (Key and Retzius, 1875; Section 4.2.2) and richly innervated (Section 4.2.3); it also contains lymph vessels (Section 4.2.4). The innervation, which has an important role in headache, has been extensively studied. Interest in immune responses in the dura and the role of dural lymph vessels, is more recent, but growing (Sections 8 and 4.2.4).

Table 2. Nomenclature for the meninges

\begin{tabular}{|c|c|c|c|}
\hline Cell layer & Preferred name & Sources & Published alternative names \\
\hline $\begin{array}{l}\text { Dural cells adjacent to the } \\
\text { calvaria }\end{array}$ & $\begin{array}{l}\text { Outer layer of } \\
\text { the dura }\end{array}$ & [1] & Internal periosteum [1], inner periosteal layer [2], endosteum [3] \\
\hline $\begin{array}{l}\text { Connective tissue between } \\
\text { the outer and inner layers of } \\
\text { the dura }\end{array}$ & $\begin{array}{l}\text { Dural connective } \\
\text { tissue }\end{array}$ & & Dura mater [4] \\
\hline $\begin{array}{l}\text { Dural cells overlying the } \\
\text { arachnoid membrane }\end{array}$ & $\begin{array}{l}\text { Inner layer of the } \\
\text { dura }\end{array}$ & & $\begin{array}{l}\text { Dural border cells [5], mesothelial cells }[6,7,8] \text {, neurothelium [8], } \\
\text { subdural neurothel of the pachymeninx, meningeal layer [2] }\end{array}$ \\
\hline $\begin{array}{l}\text { Arachnoid cells connected by } \\
\text { tight junctions }\end{array}$ & $\begin{array}{l}\text { Arachnoid } \\
\text { barrier layer }\end{array}$ & [5] & Outer layer of the arachnoid membrane [9] \\
\hline $\begin{array}{l}\text { Arachnoid cells between the } \\
\text { barrier layer and the } \\
\text { subarachnoid space }\end{array}$ & $\begin{array}{l}\text { Inner layer of the } \\
\text { arachnoid }\end{array}$ & [4] & Mesothelial cells (of the arachnoid) [6], endothelial cells [10] \\
\hline $\begin{array}{l}\text { The arachnoid barrier layer } \\
\text { together with the inner layer }\end{array}$ & $\begin{array}{l}\text { Arachnoid } \\
\text { membrane }\end{array}$ & & \\
\hline $\begin{array}{l}\text { Cells covering trabeculae and } \\
\text { bounding cisterns }\end{array}$ & Covering cells & [1] & \\
\hline $\begin{array}{l}\text { The cells below the } \\
\text { subarachnoid space, } \\
\text { continuous with the covering } \\
\text { of the trabeculae }\end{array}$ & Pial cells & [7] & $\begin{array}{l}\text { Mesothelial cells (of the arachnoid) [6] pial leptomeninx [4] } \\
\text { "As the trabeculae merge with the pia mater, these covering cells } \\
\text { spread out over this innermost membrane." [1] }\end{array}$ \\
\hline $\begin{array}{l}\text { Arachnoid membrane, } \\
\text { subarachnoid space and pia }\end{array}$ & Leptomeninges & [1] & \\
\hline $\begin{array}{l}\text { Astrocytes between the pia } \\
\text { and the neural brain }\end{array}$ & Glia limitans & & Membrana limitans gliae superficialis/vascularis (e.g., [11]) \\
\hline
\end{tabular}

1. Weed (1938). 2. Andres et al. (1987). 3. Kosaras et al. (2009) . 4. McMenamin et al. (2003) . 5. Nabeshima et al. (1975) . 6. Rodriguez-Peralta (1957) . 7. Pease and Schultz (1958) . 8. Andres (1967). 9. Oda and Nakanishi (1984) . 10. Key and Retzius (1875). 11. Krahn (1982)

4.2.1. The cell layers of the dura. The relationship of the dura to the skull can be observed in sections of decalcified tissue (Kosaras et al., 2009) which show the outer cellular layer of the dura (also called the 'inner periosteal layer' (Andres et al., 1987) or 'endosteum' (Kosaras et al., 2009; Table 2) apposed to the layer of osteoblasts (Jones et al., 1975 and Lorenzo et al., 2008). The dura has an inner layer formed by one or more layers of flattened cells called "dural border cells" by 
Nabeshima et al. (1975). Earlier authors had called them "neurothelium" or "mesothelial lining cells" (Rodriguez-Peralta, 1957; Andres, 1967), implying that they might form a barrier to diffusional exchange with the arachnoid. This is not the case: the barrier function is in the arachnoid membrane (Section 4.3; Nabeshima et al., 1975) while there are few contacts between the cells of the inner dural layer. Historically, it was suggested that a 'subdural space' could be created between the inner layer of the dura and the arachnoid membrane (Quincke, 1872 and Weed, 1923). The existence of such a space was vigorously denounced by Trolard (1892), who now appears to be correct (Waggener and Beggs, 1967; Yamashima and Friede, 1984; Frederickson, 1991; Haines, 1991). What is still sometimes referred to as the 'subdural space' is probably within the dura, between the tough, collagenous outer layers, and the mechanically weak inner layer that adheres to the arachnoid membrane (Yamashima and Friede, 1984; Haines et al., 1993). Between the outer and inner layers of the dura is further extracellular collagen associated with fibrocytes or fibroblasts (Andres, 1967; Nabeshima et al., 1975; Wei et al., 2014) and this space widens to house the meningeal arteries and their dependent vessels (Fig. 3D). A radically greater separation of the layers occurs at the sagittal cleft that separates the left and right brains. Here the inner dural layer enters deep into the cleft to form the falx cerebri, while the outer layer remains with the skull. The upper and lower boundaries of the falx cerebri house the superior and inferior sagittal venous sinuses into which drain the veins of the parietal cortex.

Intravital transcranial two-photon microscopy complements this information by observing the undisturbed, living, meninges. Strong intrinsic optical signals are produced by collagen (Figs 3G, 4B) and skull bone (Fig. 4F) by second harmonic generation (SHG; Campagnola and Loew, 2003). In mouse, intravenous injection of a non-toxic quantity of furamidine ([2,5-bis(4amidinophenyl]furan, Mathis et al., 2007) labels cell nuclei in the dura (Myburgh et al., 2013; Coles et al., 2015) and these tend to lie in two layers, corresponding to the highest densities of cells (Fig.3ABD; Coles et al. 2017). Generally similar labeling of nuclei in dural wholemounts had previously been observed in rabbits after intravenous injection of aminoacridines (RodriguezPeralta, 1957). Aminoacridines, furamidine, and other fluorescent diamidines (Mathis et al., 2006; Mathis et al., 2007) injected intravenously, also label nuclei of vascular endothelial cells, apparently of all blood vessels, including cerebral capillaries (Rodriguez-Peralta, 1957; Myburgh et al., 2013; Coles et al., 2015) (Fig. 3C,E). Apart from the vascular endothelial cells, intravenous furamidine does not readily label nuclei in the leptomeninx (Fig.3C) or the brain parenchyma (Fig.3E ), although it does label intraparenchymal nuclei if applied in superfusate to a brain slice, suggesting that furamidine is excluded by the BBB. Furamidine does not label the nuclei of all cells exposed to it: in blood, it labels a population of unidentified leukocytes, but not CD2 ${ }^{+}$lymphocytes (Text S1 in Coles et al., 2015). Hence, it is not clear if the failure of blood-borne furamidine (or aminoacridine) to label nuclei of the leptomeninx is because it does not reach the extracellular space or because it fails to enter the leptomeningeal cells.

Extracellular spaces are notoriously difficult to preserve in fixed tissue (Cragg, 1979) and are often occluded in sections of cortical dura, as in Figs. 3H,I. However, according to the texts or micrographs of several authors, the cells of the inner layers of the dura lie in large extracellular spaces (von Recklinghausen, 1862; Boehm 1869; Waggener and Beggs, 1967; Nabeshima et al., 1975; Schachenmayr and Friede, 1978; Yamashima and Friede, 1984; Oda and Nakanishi, 1984; Haines et al., 1993). These spaces contain "a loose tangle of fibrogranular connective tissue" (Frederickson, 1991) or collagen. However, in a pioneering study, Andres (1967) attributed the spaces to preparation artifacts (Fig. 3F). An indication of the geometry of the extracellular space in vivo (albeit in meningitis) was obtained incidentally when Trypanosoma brucei, the protozoan responsible for sleeping sickness, was imaged in the mouse dura. Trypanosomes, made visible by expression of fluorescent protein, or uptake of furamidine, were observed to escape from blood into the dural layer of the parietal cortex (Myburgh et al., 2013; Coles et al., 2015). Here they moved vigorously in a space extending from the underside of the skull, through the layers of collagen (Fig.4B), among fibroblasts (Fig. 4C), down to a the level including small dural vessels (Fig. 4D), but were excluded from the neighborhood of the pial vessels below the arachnoid membrane, such 
as those seen in Fig. 3BC. The trypanosomes mainly remained within volumes about $50 \mu \mathrm{m}$ across (Fig. 4EF), but were occasionally seen to move between neighboring volumes. It is not known to what extent these volumes resulted from pathological extravasation of plasma (Markowitz et al., 1987 ;

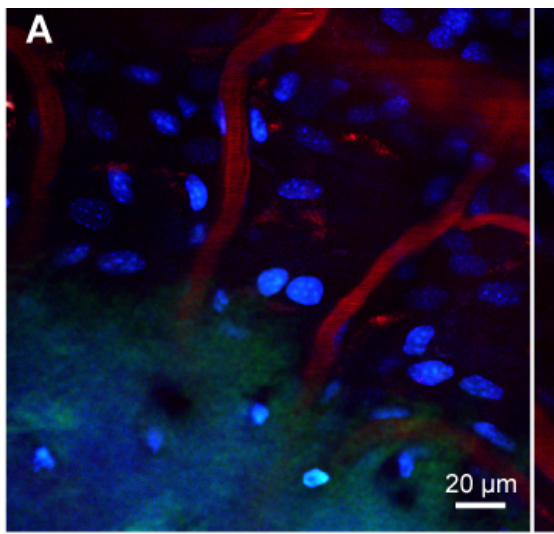

Section

4.2.3)

$$
\text { D }
$$

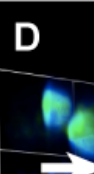

B
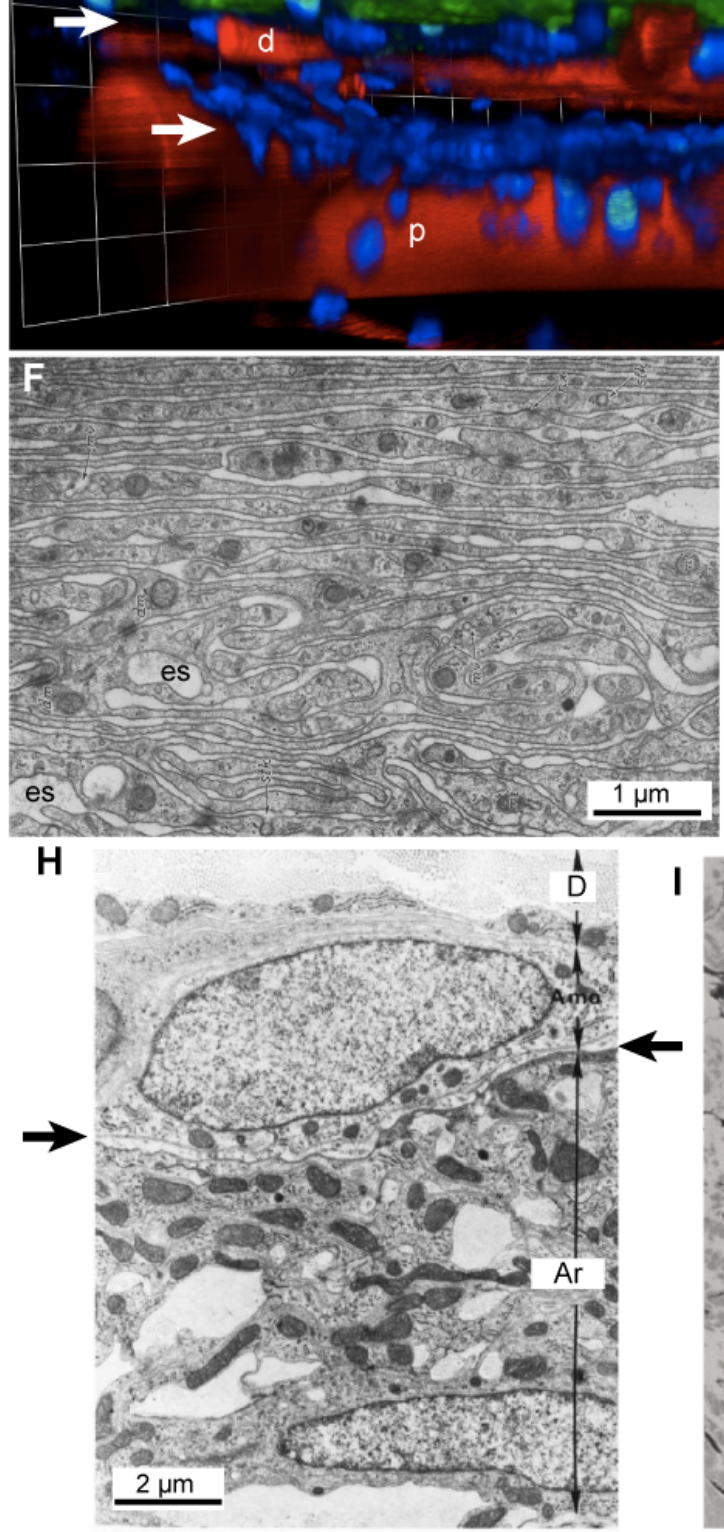

G
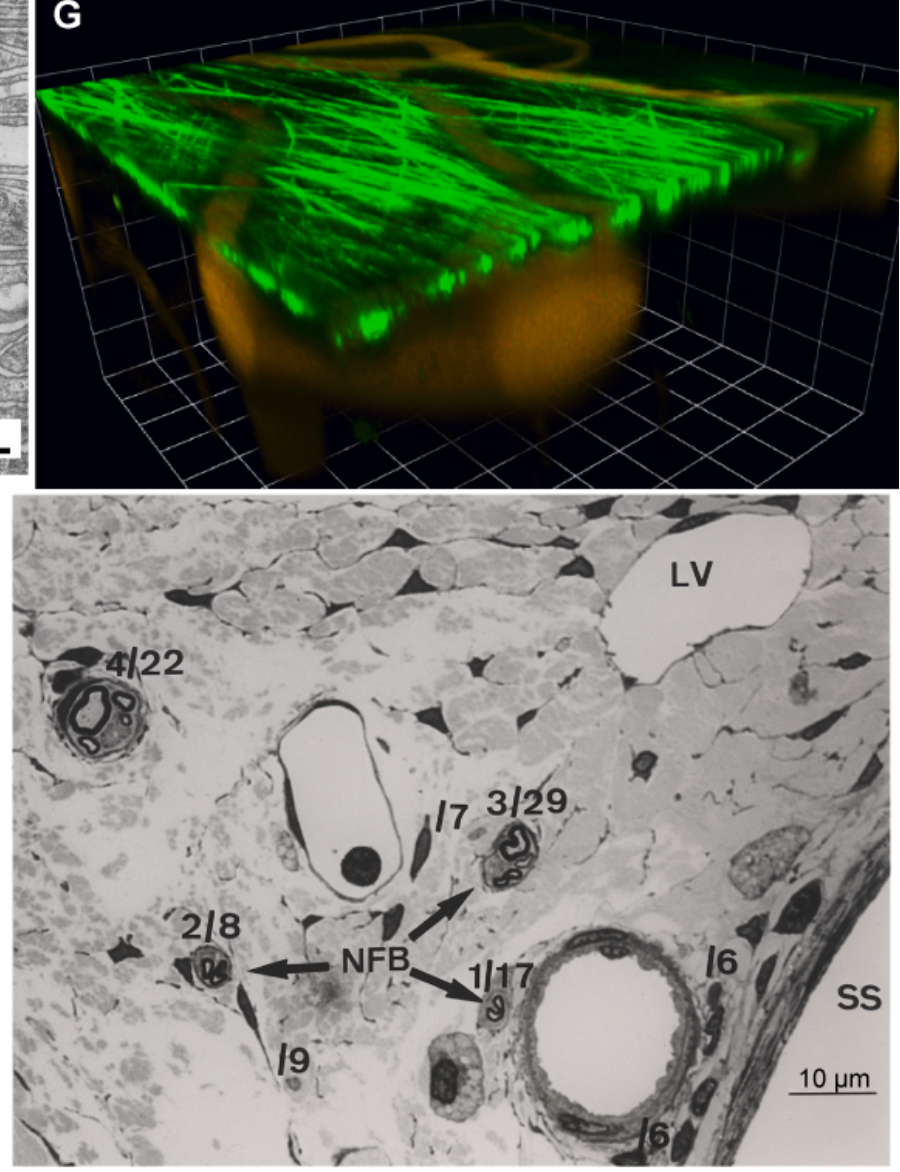
Figure 3. The anatomy of the dura. (A-D). Intravital transcranial imaging of mouse parietal cortex. Nuclei of vascular endothelial cells and a population of dural cells were labeled blue by intravenous injection of furamidine and blood plasma was labeled by Texas Red dextran $70 \mathrm{kDa}$. A slightly oblique Z-stack of images through the cortical meninges of a mouse was obtained in vivo using excitation at 800 and $1040 \mathrm{~nm}$ (see Coles et al., 2015, for methods). A. Outer dura, showing traces of skull bone (green second harmonic generation [SHG], lower left) and small blood vessels. B. Inner dura, $25 \mu \mathrm{m}$ below A. C. A plane in the leptomeninx $14 \mu \mathrm{m}$ below B. Note that i.v. furamidine labeled nuclei of vascular endothelial cells at all levels, but other cells only in the dura. D. A 3D reconstruction from the same $Z$ stack, showing skull bone (green), dural (d) and subarachnoid (p) blood vessels, and nuclei of two layers of dural cells (arrows). Grid spacing $21.3 \mu \mathrm{m}$. The upper dural layer is seen in plan view in (A) and the lower in (B). E. Intravenous furamidine labels nuclei of vascular endothelial cells throughout the brain, as here in an ex-vivo slice of mouse brain parenchyma. F. An electron micrograph of a section of dural inner layer cells of a young dog, modifed from Andres, 1967. Extracellular spaces or clefts ('Spalten') contain what Andres calls 'fine filaments or amorphous material'. Two spaces are labeled 'es'. Some smaller lettering is explained in Andres, 1967, Fig. 7 legend. G. Intravital transcranial imaging of mouse parietal meninges. Collagen (green) is made visible by SHG. Excitation wavelength $1040 \mathrm{~nm}$, grid size $21.3 \mu \mathrm{m}$, blood marker $705 \mathrm{~nm}$ Qdots. H. An electron micrograph showing the boundary (arrows) between the dura (D) and the arachnoid (Ar). Dense extracellular collagen is seen near the top of the image. Modifed from Oda and Nakanishi,1984. I. Electron micrograph of rat dura close to the superior sagittal sinus (SS) showing a blood vessel and two lymph vessels (LV). The numbers beside nerve fiber bundles (NFB) indicate (number of myelinated fibers)/(number of unmyelinated fibers). Reproduced from Andres et al., 1987, with permission from Springer Verlag. 


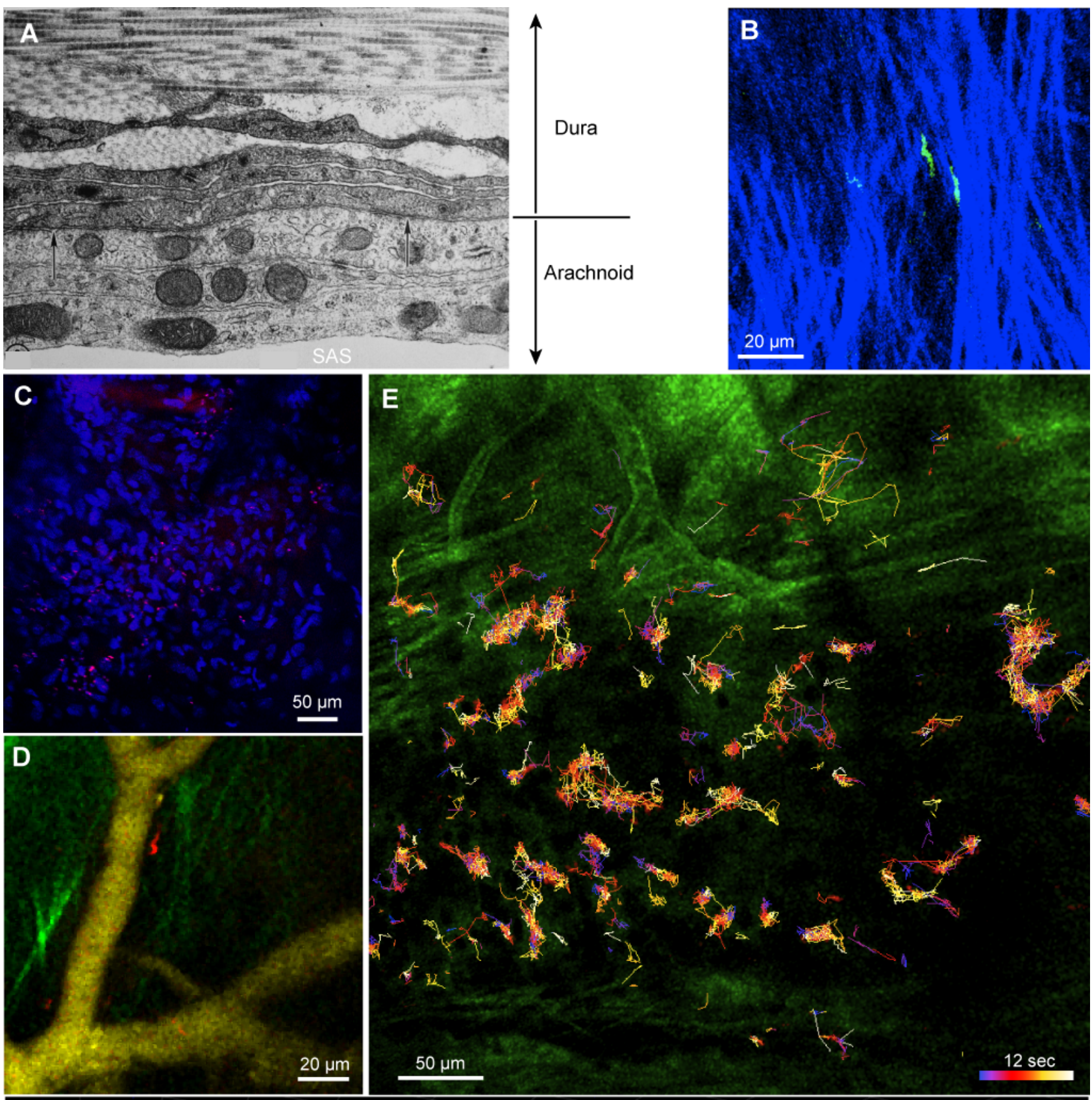

$\mathbf{F}$

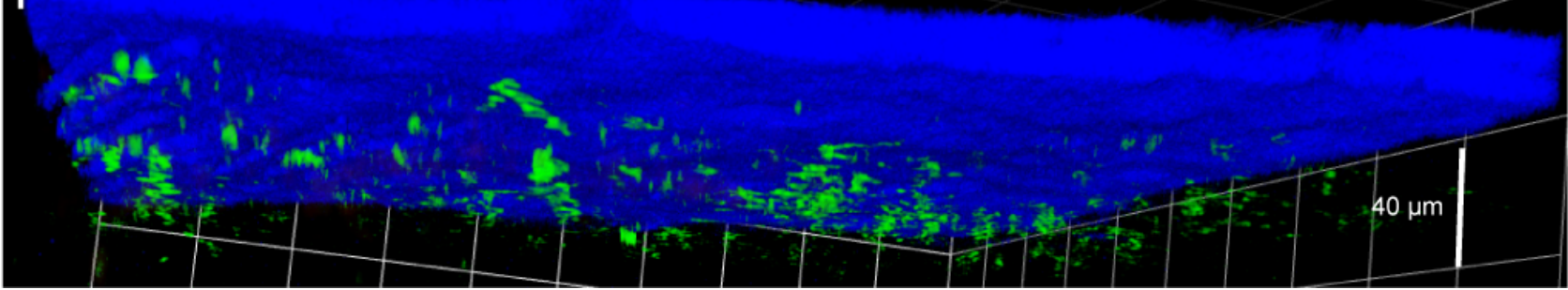

Figure 4. Extracellular space in the dura. A. Electron micrograph of guinea pig meninges showing extracellular space containing bundles of collagen. The arrows point to the boundary between the inner layers of the dura and the arachnoid barrier layer. Modified from Waggener and Beggs, 1967. B. Trypanosomes (expressing Green Fluorescent Protein) moving through extracellular collagen in the dura mater. C. Extracellular trypanosomes moving in the same focal plane as nuclei of cells of the dural connective tissue (labeled in blue by furamidine). Consequent to i.v. injection of furamidine a red dot appeared in each trypanosome, presumably corresponding to nucleic acids. D. A trypanosome (expressing mCherry) moving mainly parallel to a dural vessel. E. Trypanosomes tracks recorded over $12 \mathrm{~s}$ in the XY plane show movement mainly within areas about $50 \mu \mathrm{m}$ across. F. A time exposure of GFP-expressing trypanosomes showing their location close under the skull (blue). B-F from Coles et al., 2015. 
4.2.2 Vascularization of the dura. Blood supply to a tissue is usually matched to the tissue's requirements for metabolism, or heat exchange. The dura, despite containing much collagen, has a basal specific blood supply almost as high as that of the cerebrum: $84 \%$ in anesthetized dog (Faraci et al., 1989). Strikingly, the dural blood supply is controlled very differently from that of the cerebrum: it can increase much more (by a factor of three), and, in the case of seizures, when cerebral blood flow increases maximally, dural blood flow decreases.

Blood is supplied to the cortical dura by the meningeal arteries (Fig. 5A; Vieussens, 1684; Quincke, 1872; Key and Retzius, 1875). The main one is the middle meningeal artery, which, in the rat, has its origin from the pterygopalatine branch of the internal carotid, then courses upwards and forwards from the posterior base of the cerebrum (Fig. 5A; Keller et al., 1989). The capillary segments are shorter than $50 \mu \mathrm{m}$, and the post-capillary venules are up to $200 \mu \mathrm{m}$ long (Andres et al., 1987; Fricke et al., 2001). In man, the capillary bed is in the inner layer of the dura, where the capillaries tend to be aligned in parallel arrays (Kerber and Newton, 1973). But in Murinae the small vessels have a characteristically irregular geometry (Figs 5A,B), different from the sinuous capillaries of the parenchyma (Fig. 8A), and the larger vessels in the leptomeninx (Figs 3C, 8AB).

The dural veins over the parietal cortex drain mainly into the superior sagittal sinus (Quincke, 1872, Andres et al., 1987). There is also drainage into the middle meningeal vein (illustrated in Tsutsumi et al., 2013), some of the veins running in pairs alongside arteries (Andres et al., 1987; Shukla et al., 2002). In Homo sapiens, but not in early hominids, a pair of parietal foramina afford passage to 'emissary' veins running from the scalp into the dural sinuses (Falk, 1986; Mortazavi et al., 2012). Doppler measurements of blood flow have shown that the flow is normally very slow, but increases markedly in the inward direction when brain temperature rises (Cabanac and Brinnel, 1985), in agreement with evidence that low scalp temperature reduces brain hyperthermia (McCaffrey et al., 1975). Within the diploë (the spongy layer of the bone), tangential bony channels house the diploic veins. In man, the diploic veins are large, with an irregular and sparse geometry; they appear to have been discovered by M. Fleury, whose work was plagiarized by Dupuytren in 1803 (Jefferson and Stewart, 1928). In mouse the arborization is denser and finer (Toriumi et al., 2011). In both species, the diploic veins tend to converge into trunks leading away from the crown. Blood enters the diploic veins from small venules penetrating the walls of the bony channels, the venules being fed by numerous short arterioles that enter the bone from the scalp (Jefferson and Stewart, 1928), and, as illustrated by Zenker and Kubik (1996), also from the dura. The veins themselves also connect with vasculature in both the scalp and the dura (Cabanac and Brinnel, 1985; Toriumi et al., 2011), and, in man, can also join emissary veins (Jefferson and Stewart, 1928). When the connections to the scalp are ruptured, blood flow in vivo is (naturally) outwards (Toriumi et al., 2011), and when the mouse skull is thinned, bleeding from sites a millimeter or so apart is sometimes observed.

At least in humans, part of the venous outflow from the brain penetrates the arachnoid membrane and traverses the dura in 'bridging veins' leading to the dural sinuses (Magendie,1842; Krisch, 1988; Mortazavi et al., 2013).

As mentioned in Section 4.2.1, aminoacridines and diamidines readily pass from blood into the dura and thence to the nuclei of dural mesothelial cells, but do not cross the BBB (RodriguezPeralta, 1957; Myburgh et al., 2013; Coles et al., 2015). Similarly, horseradish peroxidase, a protein of $43 \mathrm{kDa}$ which does not cross the BBB (Reese and Karnovsky, 1967), does pass from blood into the dura, from where it is taken up into the outer layers of the arachnoid (Balin et al., 1986). The discovery of this leakiness of dural vessels is often attributed to Goldmann $(1909,1913)$, but we find no report by Goldmann of the rapid distribution of dyes injected into the blood. Instead, he followed Ehrlich (1877), and others (Bouffard, 1906; Rotky, 1912), in studying the selective affinity of cells for dyes that were injected into peritoneal, subcutaneous, or subarachnoid compartments. The leakiness of the walls of dural vessels, particularly of venules, increases further in response to certain neurotransmitters (Section 5.2.2) 

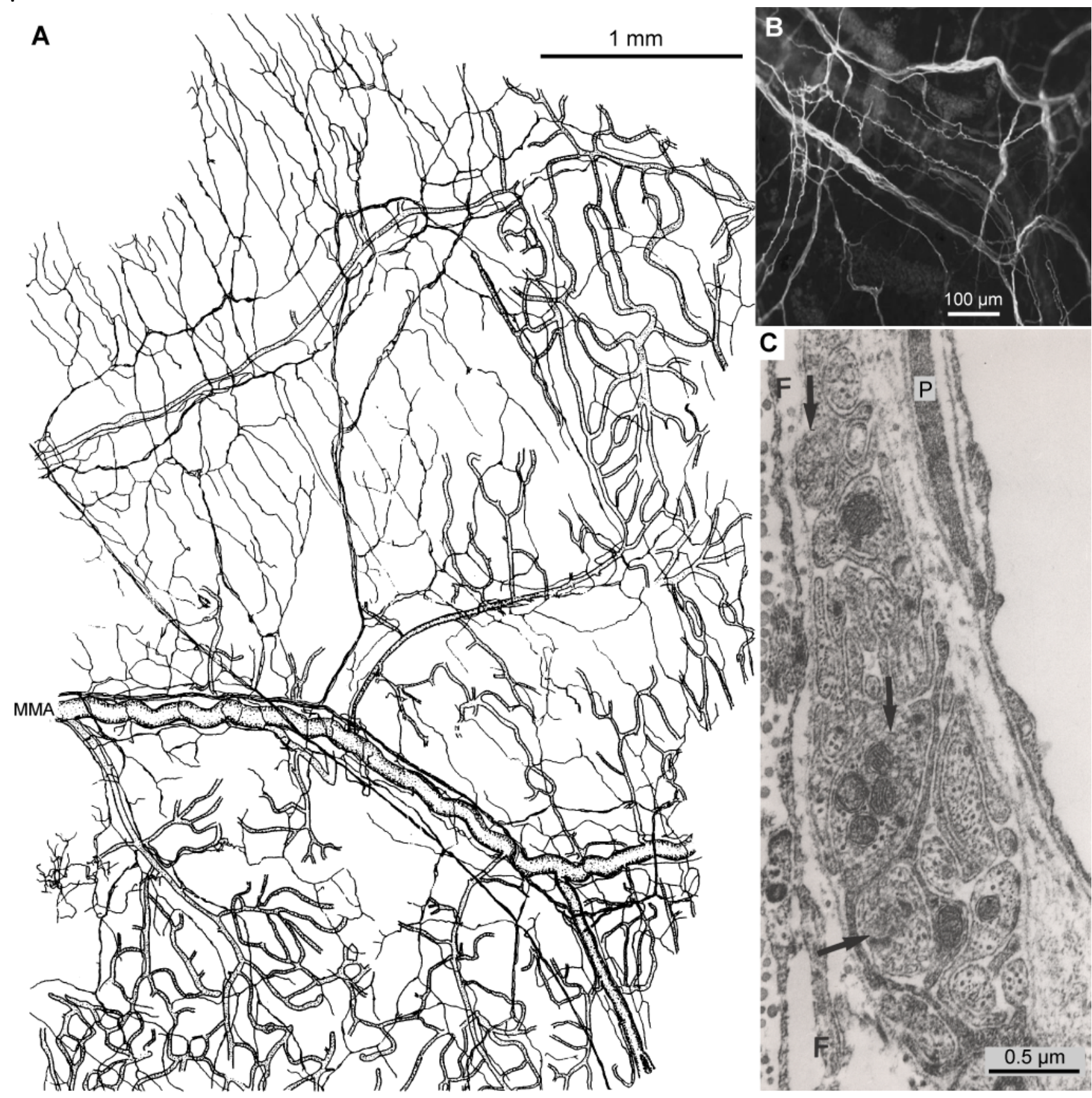

Fig. 5. Blood vessels and nerves in the rat dura. A. A camera lucida drawing of a dural wholemount showing vessels arising from the middle meningeal artery (MMA). Nerve fibers labeled by an antibody to CGRP follow the artery then innervate areas devoid of blood vessels (modifed from Messlinger et al., 1993). Orientation: occipital upwards, lateral to the left. B. Nerve fibers in the parietal dura labeled with DiI applied to the spinosus nerve as described in Schueler et al. (2014). Image kindly supplied by M. Schueler and K. Messlinger. C. Nerve terminals in the wall of a postcapillary venule. Arrows indicate axon profiles with vesicles, assumed to form neuromuscular junctions. (F) Fibrocyte. (P) Pericyte. Modifed from Andres et al., 1987.

4.2.3. Innervation of the dura. It has long been known that of the intracranial tissues it is the dura that is generally most sensitive to stimuli (Willis, 1664). Experiments on awake patients suggested that meningeal vasodilatation caused pain (Fay, 1935) and localized stimulation (electrical, mechanical, or chemical) through a craniotomy showed that a sensation of pain arises most often from nerves with endings on dural arteries, the pain often being referred to a site remote from the stimulus (Penfield, 1935; Ray and Wolff, 1940). The type of headache with the greatest health burden is migraine, a throbbing pain that can be maintained for hours or days in the absence of a 
continuing external stimulus. It was naturally expected that dural sensory innervation would play an important role in migraine and, although there is now evidence that, during the attack itself, its role is minor (Section 5.2.3), the expectation motivated many studies of dural innervation and neurotransmitters, using experiments on animals or non-invasive techniques on humans. Apart from nociception, dural innervation controls vasomotricity and can cause extravasation (Section 5), and some neurotransmitters also mediate communication with the immune system (Section 8).

The neuroanatomy of the human basal brain was illustrated in meticulous drawings by F.Wagner in 1831 (published in Arnold, 1831) and other historical contributions are summarized in Penfield and McNaughton (1940). The neuroanatomy of the rat dura is very similar to that of man (Bolay et al., 2002), the cortical dura being innervated by (nominally) sensory fibers of the fifth cranial nerve, arising from branches of the trigeminal nerve trunks (Section 4.2.3.1), and also by sympathetic (Section 4.2.3.2) and parasympathetic (Section 4.2.3.3) nerves. Alexander (1875), studying dural wholemounts of rats and mice, described two fine nerve bundles running alongside most of the dural arteries. The fibers terminate in free endings in the walls of all types of dural blood vessels, from arteries through capillaries to postcapillary venules and veins, and form a plexus innervating smooth muscle at the wall of the superior sagittal sinus (SSS; Alexander, 1875; Mayberg et al., 1984; O'Connor and van der Kooy, 1986; Andres et al., 1987; Strassman et al., 2004; Schueler et al., 2014). Keller and Marfurt (1991) were "tempted to speculate that the SSS may be the most richly innervated vascular structure in the cranial vault." As illustrated in Fig. 5A, fibers also terminate where there are no blood vessels: there are endings on collagen bundles (Andres et al., 1987; Messlinger et al., 1993), on lymph vessels leading to the SSS (Andres et al., 1987), and close to mast cells (Dimitriadou et al., 1997; Rozniecki et al., 1999; Eftekhari et al., 2013). Further branches pass through cranial sutures to innervate bone marrow and the outer periosteum (Kosaras et al., 2009; Schueler et al., 2013; Zhao and Levy, 2014).

The main techniques needed to analyze innervation (fiber tracing, immunocytochemistry of transmitters and receptors, electron microscopy of nerve terminals, and electrophysiology) are difficult to combine in a single study. It is striking that the presence of different neurotransmitters and neuropeptides can vary from one fiber to another, even though they originate in the same ganglion. The classical divisions of sympathetic (adrenergic), parasympathetic (cholinergic) and primary afferent (peptidergic), are certainly useful, but, for example, neuropeptide Y (NPY) is present in sub-populations of both sympathetic and parasympathetic neurons (Leblanc et al., 1987; Gibbins and Morris, 1988) and pituitary adenylate cyclase-activating polypeptide (PACAP) is in some parasympathetic and some primary afferent neurons (Csati et al., 2012; Eftekhari et al., 2015). The distribution of neuropeptides in dural nerve fibers can vary among species (Price and Flores, 2007), and their quantities may perhaps be labile: the proportion of axons on the circle of Willis immunoreactive to vasoactive intestinal polypeptide (VIP) that also contained NPY increased more than threefold 10-12 days after sympathetectomy (Gibbins and Morris, 1988). It is not known if there is switching of transmitters in the dura as complete at that found in central synapses (Spitzer, 2015).

The following sections on the three basic systems focus on the supratentorial dura of Murinae. Further bibliography, including on man, can be found in Luschka, 1850; Alexander, 1875; Steiger et al., 1982; Mayberg et al., 1984; O'Connor and van der Kooy, 1986; Markowitz et al., 1987; Ruskell, 1988, and Schueler et al., 2014.

4.2.3.1 Trigeminal innervation of the dura. The trigeminal nerve (or cranial nerve $\mathrm{V}$ ) has three large divisions running forwards from the trigeminal ganglion: the ophthalmic (V1), maxillary (V2) and mandibular (V3), and a small part of this system innervates the dura. In the rat, the rostral supratentorial dura is innervated by two branches of the ophthalmic division, the ethmoidal and tentorial nerves (Andres et al., 1987). The ethmoidal, which is a continuation of the nasociliary nerve after it passes through the ethmoidal foramen (Bove and Moskowitz, 1997) innervates the dura of the olfactory bulbs and the rostral superior sagittal sinus. The tentorial nerve innervates the dura of the rostral lateral convexities (Andres et al., 1987). Offshoots of the mandibular division 
(V3), the nervus spinosus, accompany the MMA and send fascicles through the dura upwards and forwards from the tentorium (Schueler et al., 2014). In contrast, in the mouse, Huang et al. (2012) find that dural innervation originates from V2 rather than V3 (as well as V1). Altogether, the trigeminal innervation of the rat dura consists of some 250 myelinated and 800 unmyelinated fibers (Andres et al., 1987). Some trigeminal neurons (in cat) send fibers to both the dura and the MCA (O'Connor and van der Kooy, 1986).

The great majority of trigeminal neurons that innervate skin and the minority that innervate the dura show many functional similarities. Most respond to several kinds of noxious stimuli, such as heat, cold, acidity, and mechanical distortion (Bove and Moskowitz, 1997). As is usual in nociceptive fibers (e.g., Dai, 2016), ion channels of the transient receptor potential (TRP) family are expressed (Shimizu et al., 2007; Price and Flores, 2007; Huang et al., 2012) and also acid-sensing inward current channels (ASICs; Yan et al., 2013). Far from simply initiating afferent nerve impulses, most, perhaps all, of the nerve fibers contain, release, and respond to a range of neurotransmitters, notably neuropetides.

The neuropeptide present in the greatest number of dural fibers of trigeminal origin appears to be calcitonin gene related peptide (CGRP). Within the rat cranium, CGRP is largely restricted to the trigeminal ganglion (Rosenfeld et al., 1983; Lee et al., 1985b) where it is present in a proportion of the neurons ranging from 23\% (O'Connor and van der Kooy, 1988) to about 50\% (Lee et al., 1985a; Reuss et al., 1992; Eftekhari et al., 2015). In the mouse, the proportion of dural nerves containing CGRP may be lower than in the rat (O'Connor and van der Kooy, 1988; Uddman et al., 1989; Huang et al., 2012). In the rat dura, CGRP is present in an extensive network, in bundles of small, unmyelinated, fibers which accompany arteries and their branches, usually ramify into areas away from blood vessels, and form plexuses on the superior sagittal sinus (Fig. 5A; Keller and Marfurt, 1991; Messlinger et al., 1993; Shimizu et al., 2007; Eftekhari et al., 2013).

Substance P (SP), the first neuropeptide to be found in nociceptive neurons, is present in the rat trigeminal ganglion (Hökfelt et al., 1975; Uddman et al., 1989), where is often colocalized with CGRP (Gibbins et al., 1985; Reuss et al., 1992; Ma et al., 2001). In the trigeminal projection to the MMA, about half of the CGRP neurons also contain SP (O'Connor and van der Kooy, 1988). In dural wholemounts, the pattern of SP labeling is very similar that of CGRP (von Duering et al., 1990; Keller and Marfurt 1991;Messlinger 1993), suggesting that CGRP fibers lacking SP mainly innervate arteries. Almost no cell bodies containing SP or CGRP are reported in the superior cervical ganglion, although they may be present in a few axons passing through it (Gibbins et al., 1985; Tsai et al., 1988; Uddman et al., 1989). A third neuropeptide, cholecystokinin (CCK), is found in trigeminal projections to the MMA but not elsewhere in the dura (O'Conner \& van der Kooy 1988). Also present in some trigeminal neurons are pituitary adenylate cyclase activating polypeptide (PACAP; Moller et al., 1993; Eftekhari et al., 2015) and the peptide neurokinin A (NKA; Edvinsson et al., 1988). All five of these neuropeptides, CGRP, SP, CCK, PACAP and NKA, can cause vasodilatation of pial or meningeal arteries (Section 5). SP, but not CGRP, causes extravasation in the rat dura (Markowitz et al. 1987).

Receptors to CGRP are found in the walls of dural blood vessels (Lennerz et al., 2008; Eftekhari et al., 2013) and, as shown by Eftekhari et al. (2013) on mast cells. On nerves, Lennerz et al. (2008) find the receptors on Schwann cells rather than the axons themselves. The sensitivity of fibers to mechanical stimuli is increased for tens of minutes by inflammatory mediators (Strassman et al., 1996) including CGRP and SP (Devesa et al., 2014). This sensitization may in part be caused by $\mathrm{Ca}^{2+}$-dependent exocytosis of vesicles that liberate CGRP and other mediators, which then increase the responses to stimulation of nerve fibers and mast cells (Devesa et al., 2014).

To a first approximation, nociception by trigeminal fibers in the dura is in keeping with the classical synaptic anatomy of the trigeminal ganglion, which dictates that the fibers innervating the meninges are afferent. However, trigeminal fibers also have efferent functions. This is seen clearly in work by Schueler et al. (2013) in which stimulation of extracranial endings caused release of CGRP in the dura. The mechanism of this is presumably the 'axon reflex', well known in skin, whereby depolarization of one sensory ending evokes action potentials that, in addition to travelling 
towards the sensory ganglion, antidromically invade axon collaterals (Bruce, 1913; Wårdell et al., 1993). Similarly, electrical or mechanical stimulation of the dura itself increases blood flow in the dura (Kurosawa et al., 1995; Bolay et al., 2002) and this reflex is not abolished by sectioning of the trigeminal nerve trunk (including parasympathetic pathways; Bolay et al., 2002). Concerning the proximal nerve segments leaving the trigeminal nucleus towards the dura, there seems to be no direct experimental demonstration of the direction of endogenous impulse traffic. At the onset of a migraine attack, cortical activity both excites trigeminal neurons and increases dural sensitivity (Noseda et al., 2010; Pietrobon and Moskowitz, 2014). Parasympathetic pathways play a role (Section 4.2.3.2; Bolay et al., 2002) but the idea seems to have slipped into the literature that, as Pietrobon and Moskowitz (2014) say, there are "impulses transmitted antidromically" along trigeminal fibers (Pietrobon and Moskowitz, 2014), as previously implied by Dimitriadou et al. (1991), Bolay et al. (2002), Edvinsson and Uddman (2005) and Huang et al. (2012).

4.2.3.2 Sympathetic innervation of the dura. The sympathetic transmitter norepinephrine (noradrenaline) tends to counteract dural inflammation (Reynier-Rebuffel et al., 1997), and a defective noradrenergic system correlates with increased susceptibility to migraine (Gotoh et al., 1984; Sokolov et al., 2013). In rat, Uddman et al. (1989) examined several candidate ganglia for retrograde labeling from the MMA and found that at least half of the fibers terminating on the MMA are sympathetic, originating in the superior cervical ganglion and accompanying the tentorial nerve. There is also "extremely dense" innervation from the superior cervical ganglion to the walls of the anterior superior sagittal sinus and neighboring dura, where some endings are not on blood vessels (Keller et al., 1989). Histology (for monoamines and for dopamine- $\beta$-hydroxylase, the enzyme converting dopamine to norepinephrine) indicate fibers containing norepinephrine (Edvinsson and Uddman, 1981 and Keller et al., 1989). There is also innervation by fibers immunoreactive to antibodies against neuropeptide Y (NPY; Edvinsson et al., 1987; von Duering et al., 1990; Keller et al., 1991). Their presence is greatly reduced by superior cervical ganglionectomy (Edvinsson et al., 1987; Keller et al., 1991), which suggests that the fibers are sympathetic, but NPY is also present in parasympathetic neurons (Section 4.2.3.3). There is one report of fibers containing serotonin $(5 \mathrm{HT})$, present in 'small to moderate numbers' (Keller and Marfurt, 1991), and terminating mainly in the walls of arteries.

4.2.3.3. Parasympathetic innervation of the dura. Fiber tracing from the middle meningeal artery (MMA) in rat shows innervation from parasympathetic ganglia: the sphenopalatine (pterygopalatine) and the otic ganglia, and the jugular-nodose ganglionic complex (Uddman et al., 1989). Histological evidence for the predominant parasympathetic neurotransmitter, acetylcholine, is usually obtained by labeling its synthesizing and degrading enzymes. In guinea pig dura, acetylcholinesterase has been reported by Amenta et al. (1980) and Edvinsson and Uddman (1981). The latter describe "a prominent system" of acetylcholinesterase nerve fibers around the dural blood vessels, "as well developed as that of the NA-containing fibres and having a very similar localization". In rat, choline acetyltransferase immunoreactivity has been found in all neurons of the otic ganglion, and most neurons in the sphenopalatine (Leblanc et al., 1987). Neuropeptide immunoreactivity found in cranial parasympathetic ganglia suggests the presence of neuropeptide $Y$ (NPY; Leblanc et al., 1987), Pituitary Adenylate Cyclase-Activating Polypeptide (PACAP; Csati et al., 2012) and vasoactive intestinal polypeptide (VIP; Leblanc et al., 1987; Csati et al., 2012)). NPY and VIP are found in extracts of human MMA (Jansen, 1992). NPY immunoreactivity is found in the dura (von Duering et al., 1990) where most of it is eliminated by sympathectomy (Edvinsson et al., 1987; Keller et al., 1991). Sparse immunoreactivity for VIP has been reported in rat supratentorial dura (Eftekhari et al., 2013).

4.2.4. Lymph vessels in the cortical dura. Two recent studies by Aspelund et al. (2015) and Louveau et al. (2015), which used fluorescence microscopy to describe lymph vessels in the dura, have led to debate about credit for their discovery. Dural lymph vessels were described in humans 
by Mascagni in 1787 (reproduced in Bucchieri et al., 2015), and by von Recklinghausen (1862). But dural lymph vessels are not normally conspicuous and, although Boehm (1869), for example, discussed them, neither he nor Key \& Retsius (1875) indicate them in their figures; despite searching for them, von Recklinghausen did not find them in the guinea pig. Jacobi (1924) claimed to show dural lymph vessels, but we find his images unconvincing. When lymph vessels were not fully visualized, stomata, like those in peripheral lymph capillaries, were sometimes taken as indicative of their presence (Iwanow and Romodanowsky, 1928). Schwalbe (1869) had shown that particles can be carried from CSF to the nasal mucosa and cervical lymph nodes (see Section 6.2), thereby showing a connection between subarachnoid space and extracranial lymphatics. Then Zwillinger (1912) injected lipophilic dye in the subarachnoid space close to the cribriform plate of human cadavers and, after $24 \mathrm{~h}$, found the dye had labeled a network of vessels within the meninges, as well as in the nasal mucosa, and he identified these as lymph vessels.

In dog, dural lymph vessels leading out of the basal brain by the jugular foramen were described by Földi et al. (1966). In the cortical dura, 'blood vessels, lymphatics and nerve fasciculi ..... grouped in focal aggregates' were observed in rat by Waggener and Beggs (1967), and electron micrographs by Andres et al. (1987) show unambiguous lymph vessels (Fig.3I). Visualization of marker molecules specific to lymphatic endothelial cells has made possible a full description of the lymphatic network of the mouse cerebral dura (Aspelund et al., 2015). Over the parietal cortex, lymph capillaries are found beside the blood vessels of the sagittal sinus and the middle meningeal artery, but are absent from large areas. These lymph vessels exit the cranium through the cribriform plate and with the middle meningeal artery (Furukawa et al., 2008; Aspelund et al., 2015). Elsewhere, lymph vessels leave the cranium with nerves and blood vessels, the jugular foramen being a major route (Földi et al., 1966; Aspelund et al., 2015). The functionality of the dural lymphatics has been supported by the effects of ligating the cervical lymph vessels, which causes edema in the pia (Földi et al., 1963), cognitive impairment (Foldi et al., 1968) and swelling of cortical dural lymph vessels (Aspelund et al., 2015; Louveau et al., 2015).

A lymph vessel in the dura is presumably surrounded by the interstitial fluid of the dura, which is not in communication with CSF (e.g., Key and Retzius, 1875; Penfield, 1923; RodriguezPeralta, 1957). However, Indian ink (Kida et al., 1993a) and T lymphocytes (Louveau et al., 2015) appear to pass from the leptomeninx into the dural lymphatics. The anatomical substrate of this passage is not evident, and Louveau et al. present a scheme in which $\mathrm{T}$ cells simply pass through the arachnoid membrane (their ED Fig.10). Possibly, pathways through the arachnoid membrane might be associated with the extensions of the SAS that traverse the dura to the arachnoid villi, and allow CSF to flow into blood (von Duering and Andres 1991; see Section 6.2), or with the bridging veins (Magendie,1842; Krisch 1988; Mortazavi et al 2013).

Most authors describe no conventional lymph vessels in the leptomeninx or the healthy neural brain (Aspelund et al., 2015; Louveau et al., 2015), although lymphoid aggregations are present in the leptomeningeal extensions to the arachnoid granulations (von Duering and Andres, 1991). However, many authors have pointed out that the perivascular spaces may serve similar functions (His, 1865; Kölliker, 1867; Schwalbe, 1869; Zwillinger, 1912; Prineas, 1979; Iliff et al., 2012; see Section 6.4). An exception is the report by Prineas et al. (1979) of "thin-walled channels observed in perivascular spaces in unaffected CNS tissue ... indistinguishable from lymphatic capillaries in other tissues in terms of both their structure and contents" (Prineas et al., 1979). In certain forms of EAE, tertiary lymph nodes develop in the brain (Kuerten et al., 2012).

\subsection{The leptomeninx}

An impermeable barrier separates the arachnoid from the dura (Key and Retzius, 1875; Weed, 1923; Rodriguez-Peralta, 1957) and is accounted for by the outer layer of arachnoid cells, which are connected by tight junctions (Nabeshima et al., 1975). The barrier cells express several drug transporter genes that are also expressed at the blood-brain barrier, including p-glycoprotein on the apical (dura-facing) membrane (Yasuda et al., 2013). Below the barrier layer there is at least one layer of inner arachnoid cells (Pease and Schultz, 1958; Nabeshima et al., 1975; see Table 1) 
overlying a real or virtual subarachnoid space (see below). These barrier and non-barrier arachnoid cells constitute the 'arachnoid membrane'. Those of the inner layer have numerous mitochondria (Pease and Schultz, 1958; Oda and Nakanishi, 1984) and the proximity of the rich capillary network of the inner layers of the dura might permit intense metabolic activity by the arachnoid membrane. In places, trabeculae, consisting of bundles of collagen covered with extensions of arachnoid cells, extend through the subarachnoid space (Key and Retzius, 1875; Weed, 1923; Pease and Schultz, 1958; Andres, 1967). However, the presence in thin $(1 \mu \mathrm{m})$ tissue sections of continuous structures traversing the subarachnoid space (of man) suggests that membranous septa are more common than filiform trabeculae (Alcolado et al., 1988; Weller, 2005), and the 'cisterns' described by Pease and Schultz (1958) would indeed require sheets of tissue.
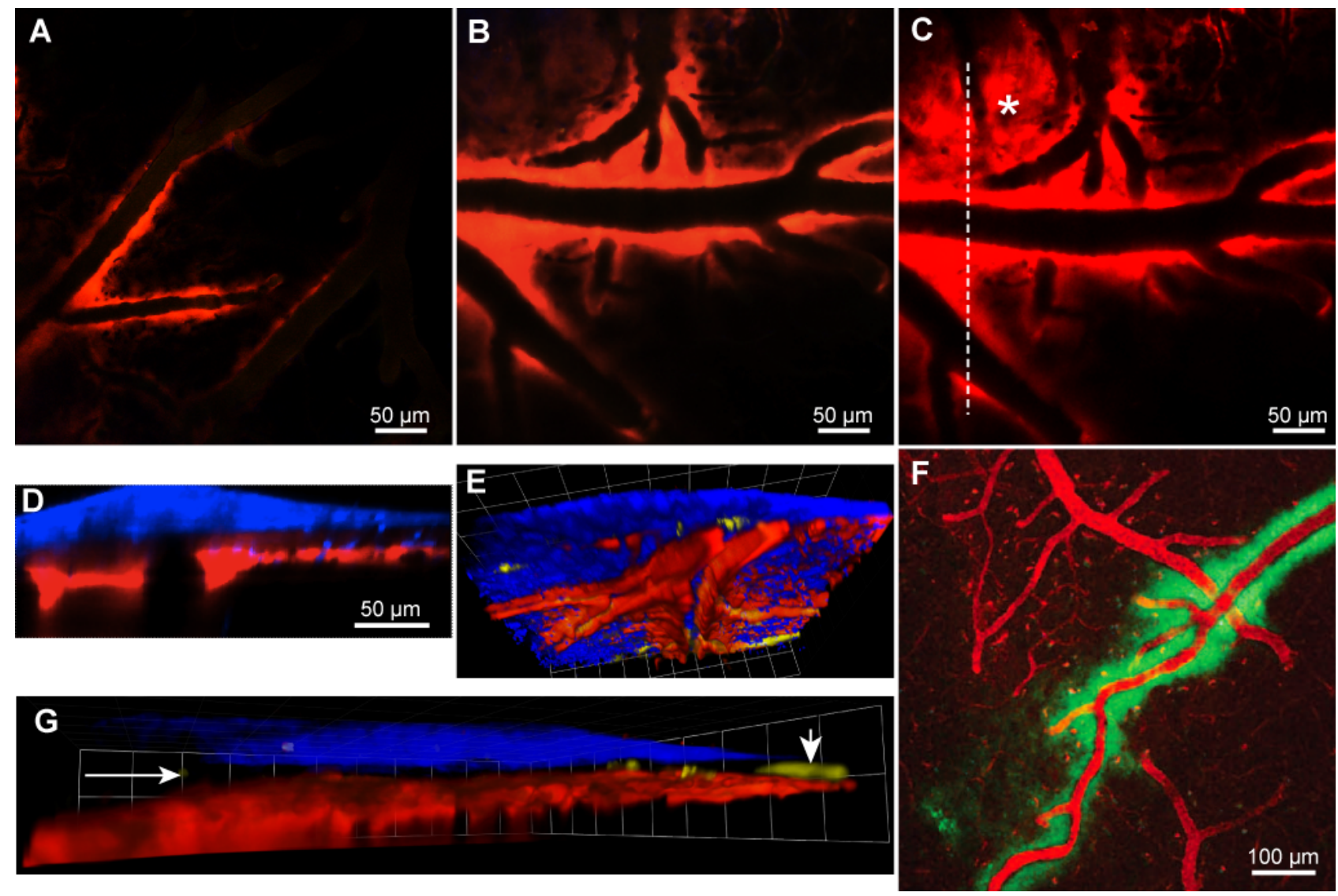

Fig. 6. Subarachnoid space in mouse. A-G. Texas Red dye was infused in the cisterna magna and its arrival in the cortical meninges observed at $19 \mathrm{~min}(\mathbf{A}), 42 \mathrm{~min}(\mathbf{B}$, in a different field) and $64 \mathrm{~min}(\mathbf{C})$. The asterisk in $(\mathbf{C})$ indicates a region accessed by the dye only at the later time. D. An XZ section along the dashed line in (C) (seen from the right). The blue is a second harmonic signal (SHG) from skull bone. E. A 3D reconstruction viewed obliquely from below showing Texas Red labeling confined to paravascular spaces $59 \mathrm{~min}$ after infusion. F. Pial vessels labeled with fluorescent dextran (red). $2000 \mathrm{kDa}$ fluorescent dextran (green) infused in the cisterna magna reached channels adjacent to an artery in 5 min. From Iliff et al 2012, by permission . G. A 3D reconstruction showing an unlabeled layer (long arrow) between the subarachnoid space (red) and the skull (blue). Dural vessels (yellow, short arrow) are present in this layer. $30 \mathrm{~min}$ after infusion in the cisterna magna.

At the lower bound of the subarachnoid space, the arachnoid cells are renamed pial cells, which extend in one (Pease and Schultz, 1958) or several (Morse and Low, 1972) layers over a basal lamina (Bifari et al., 2009). Bifari et al. report that the pia contains nestin-positive cells showing properties of neuronal stem cells; Nakagomi et al. (2015) find neural progenitors in the pia after ischemic trauma. The pial layer is fenestrated, and particles as large as colloidal gold complexed with albumin readily cross it to reach a sub-pial space above the layer of astrocyte endfeet (the glia limitans; Ichimura et al., 1991). Arteries, arterioles, veins and venules, are present, most of them lying in grooves in the surface of the parenchyma, but there are no capillaries in the 
subarachnoid space or the pia mater (Weed, 1914a and Fricke et al., 1997).

Published drawings have repeatedly featured long trabeculae crossing a voluminous subarachnoid space (e.g., Fig. 1A). Trabeculae are certainly present in large mammals in places where, as Weed (1914) says: "the subarachnoid space over the cerebral hemispheres extends deeply into the cerebral sulci, while the arachnoid membrane bridges these furrows from gyrus to gyrus". Long trabeculae are also present on the ventral surface of rat brain, as shown in Fig.1 of Fricke et al. (1997). However, concerning the dorsal cortex (of large mammals) Weed continues: "Over the convolutions the subarachnoid space is only of capillary thickness until it dips again into a fissure" (Weed, 1914a). In Murinae, the cortex lacks sulci and electron microcroscopy has repeatedly shown that the subarachnoid space is thin or virtual over most of the dorsal cortex (Pease and Schultz, 1958; Morse and Low, 1972; Nabeshima et al., 1975; Oda and Nakanishi, 1984). Weller and co-workers (Laman and Weller, 2013) state that "in rodents, the subarachnoid space containing CSF over the surface of the brain is restricted to channels alongside the major cerebral arteries". In vivo imaging of dyes infused in the cisterna magna would extend this to include channels alongside smaller surface arteries (Fig. 6A-F; Iliff et al., 2012; Coles et al., 2015). The ventral surface of the brain is different; there, the subarachnoid space in the vicinity of the circle of Willis is capacious (Kida et al., 1993a; Fricke et al., 2001).

Evidence that large molecules and cells can move from the leptomeninx down the perivascular space of penetrating vessels was given in Section 3.2.2. Recent two-photon imaging appears to support circulation of CSF between the subarachnoid space and the perivascular spaces and will be discussed in Section 6.4.

4.3.1. Innervation of the leptomeninx. Kölliker (1867) mentions perivascular nerves on pial vessels, and Huber (1899) used intravital methylene blue to stain plexuses of myelinated and unmyelinated fibers extending to small pial arteries. In man, nociceptive stimulation of the large arteries of the circle of Willis is painful, but the pial arteries and veins over the cortex, and connective tissue of the cortical leptomeninx are generally insensitive (Ray and Wolff, 1940). Unlike the dura, the nerve endings in the leptomeninx seem to be almost exclusively on the vessels, in the "adventitial leptomeninx" (Fricke et al., 1997). In rat, pial arteries receive fibers from the trigeminal sensory ganglion (O'Connor and van der Kooy, 1986; Saito et al., 1987)) and immunohistochemistry shows fibers reactive to neuropeptides characteristic of trigeminal neurons. These include CGRP (O'Connor and van der Kooy, 1988; Tsai et al., 1988; Price and Flores, 2007), Neurokinin A (NKA; Edvinsson et al., 1988) and substance P (Uddman et al., 1981; Liu-Chen et al., 1983), and also vasodilatation mediated by receptors to CGRP (Edvinsson et al., 1987; Petersen et al., 2005), NKA (Edvinsson et al., 1988) and SP (Edvinsson et al., 1981; Edvinsson et al., 1988). As a result of this sensory innervation, perfusion of the subarachnoid space with inflammatory mediators or autologous blood plasma activates second-order neurons in the trigeminal nucleus caudalis (Ebersberger et al., 1997; Ebersberger et al., 1999). However, there is greater innervation of pial vessels by sympathetic and parasympathetic fibers. Vasodilating parasympathetic innervation was described by Chorobski and Penfield (1932) and supported by identification of fibers containing acetylcholinesterase (Edvinsson et al., 1972), vasoactive intestinal peptide (VIP; Kobayashi et al., 1983 and Matsuyama et al., 1983), pituitary adenylate cyclase activating peptide (PACAP; Uddman et al., 1993; Baun et al., 2011), substance P (SP;(Saito et al., 1987; Fricke et al., 1997) or nitric oxide synthase (Fricke et al., 1997). Of these, VIP and PACAP may be the main vasodilators (Suzuki et al., 1988; Baun et al., 2011). The parasympathetic innervation has been traced to the otic and sphenopalatine ganglia and the jugular-nodose ganglionic complex (Uddman et al., 1989). Sympathetic fibers containing norepinephrine (Falck et al., 1965; Nielsen and Owman, 1967), or its synthetic enzyme, dopamine $\beta$ hydroxylase (Fricke et al., 1997), and neuropeptide Y (Edvinsson et al., 1987), are also present.

\section{Vascular dynamics in the meninges.}

5.1. Control of pial vessel diameter. The view of Bayliss et al. (1895) that pial arteries normally do little to control cerebral blood flow, because compensation is made in other organs to maintain a 
constant supply to the brain, is, by and large, correct. It is now well established that the changing demands of physiological brain metabolism are met locally, by regulation of arterioles and capillaries in response to activity in nearby groups of neurons (Peppiatt et al., 2006; Gordon et al., 2007; Iadecola and Nedergaard, 2007; Attwell et al., 2010). Physiological activation of neurons (e.g., by moving whiskers) can lead to a modest increase in flow through the overlying pial arteries, which are observed to dilate somewhat (Cox et al., 1993). Part, or all, of this dilatation may be due to reduced myogenic tone in response to the reduced downstream resistance (Bayliss, 1902; Nelson et al., 1995). However, $\mathrm{Xu}$ et al. (2008) report that intense and widespread neuronal seizure increased pial artery diameter by as much as $30 \%$. This increase was reduced by meningeal application of a blocker of astrocyte gap junctions, suggesting that a network of astrocytes was involved in signaling to pial arteries. Histological evidence of autonomic innervation of pial arteries (which is dense on the circle of Willis) led to much study of control of cerebral blood supply by sympathetic and parasympathetic nerves (reviewed in Forbes and Cobb, 1938, and Nelson and Rennels, 1970). As expected, parasympathetic and sympathetic stimulation respectively cause vasodilatation and, usually, constriction, although the effects are modest (Forbes and Wolff, 1928; Forbes and Cobb, 1938; Nelson and Rennels, 1970; Strandgaard and Sigurdsson, 2008). More selective stimulation of parasympathetic fibers from the sphenopalatine ganglion in rat increased pial blood flow (measured by laser-Doppler flowmetry) by up to a maximum of $47 \%$ (Suzuki et al., 1990b). Stimulation of the cervical sympathetic trunk has very variable effects on pial arteries, with constriction being most commonly reported (Forbes and Wolff, 1928; Kobayashi et al., 1971). Normally, the role of sympathetic control is minor (Strandgaard and Sigurdsson, 2008; ter Laan et al., 2013) but it may be important in "severely challenging circumstances, such as delayed cerebral ischaemia after subarachnoid haemorrhage" (ter Laan et al., 2013). Stimulation of the trigeminal cerebrovascular nerves in rat caused a small $(<20 \%)$ and transient increase in pial blood flow (Suzuki et al., 1990a).

In contrast to the small and variable neurogenic effects, the dilatation of pial arteries by hypercapnia is marked and consistent (Wolff, 1930; Nelson and Rennels, 1970). The causes of this are still debated: acidification of blood alone has no effect (Harper and Bell, 1963), but acidification of CSF does (Lassen, 1968). Possible mechanisms involving $\mathrm{pCO}_{2}$, prostaglandin $\mathrm{I} 2\left(\mathrm{PGI}_{2}\right.$ or prostacyclin), $\mathrm{H}_{2} \mathrm{~S}, \mathrm{NO}$, endothelium-derived hyperpolarizing factor (EDHF) and endothelin-1 (ET1), as well as $\mathrm{pH}$, are reviewed in Yoon et al. (2012).

5.2.1. Vasomotor control in the dura. Unlike pial arteries, the middle meningeal artery responds vigorously both to neural input and to factors released from mast cells. The value to the animal of this vasomotricity is not clear, although it has been suggested that changes in blood flow through the dura help to control the temperature of the brain (Zenker and Kubik, 1996). The diameters of the larger dural vessels in Murinae are readily observed in vivo through the thinned skull (Williamson et al., 1997a), and can be measured by magnetic resonance angiography in humans (Nishimura et al., 1986). Control is by a combination of at least five systems, some of which involve multiple neurotransmitters. Like most small arteries and arterioles, the MMA constricts in response to increased luminal pressure, with no involvement of extrinsic innervation (Syed et al., 2012). Application of the sympathetic transmitter norepinephrine also causes constriction (Ray and Wolff, 1940; Edvinsson et al., 1998a), as does NPY, which is present in both sympathetic and parasympathetic neurons (Edvinsson et al., 1998a). The parasympathetic transmitter VIP, relaxes isolated MMAs, but with an $\mathrm{EC}_{50}$ a thousandfold greater than that of pituitary adenylate cyclaseactivating polypeptide (PACAP38; Syed et al., 2012), which is found in neurons of both parasympathetic ganglia (Csati et al., 2012; Eftekhari et al., 2015) and the trigeminal ganglion (Syed et al., 2012; Eftekhari et al., 2015). The other major neuropeptides found in trigeminal neurons also cause relaxation when applied to the dura in vivo or to isolated lengths of the MMA: CGRP ((Kurosawa et al., 1995)Petersen et al., 2004(Schwenger et al., 2007)), and SP ((Edvinsson et al., 1981)Jansen et al., 1992) and are likely involved in neuromuscular transmission. Applied intraluminally, SP (Faraci et al., 1989; Williamson et al., 1997a), NKA (Williamson et al., 1997a), 
and serotonin (5-HT); Faraci et al., 1989) dilate, as do adenosine (Faraci et al., 1989), histamine (Akerman et al., 2002c) and NO donors (Messlinger et al., 2000; Akerman et al., 2002a). Intravenous injection of CGRP was less effective than SP (Williamson et al., 1997a), but CGRP is clearly important: in a preparation of the dura attached to the hemiskull, trigeminal stimulation released CGRP but not SP (Ebersberger et al., 1999a). Given that dural vasodilatation was long thought to contribute to the generation of migraine, it is surprising that we find no unequivocal reports of stimulation of ganglia or nerve tracts on blood flow or arterial diameter in the dura. Transcranial laser speckle imaging (Bolay et al., 2002; Ayata et al., 2004) or careful use of Doppler flowmetry (Kurosawa et al., 1995) allows separate measurements in the MMA and in pial vessels of a parameter related to blood flow (Fercher and Briers, 1981; Kazmi et al., 2015). Stimulation with electrodes placed either on the dura (Kurosawa et al., 1995; Akerman et al., 2002b; Wang et al., 2014) or on the skull (Williamson et al., 1997b) does increase the diameter of the MMA or blood flow. This effect can be reduced or blocked by antagonists of CGRP receptors (Kurosawa et al., 1995; Williamson et al., 1997b) and so is probably caused by activation of trigeminal terminals and the axon reflex. The chemical pathways leading to neurogenic vasodilatation are further discussed by Schwenger et al. (2007). In addition, mast cells are involved. Most CGRP-containing nerve fibers terminate far from vessels and often run adjacent to some of the mast cells (Messlinger et al., 1993; Dimitriadou et al., 1997; Strassman et al., 2004), which have receptors to CGRP (Lennerz et al., 2008; Eftekhari et al., 2013) and degranulate on stimulation of the trigeminal nerve (Dimitriadou et al., 1991). Infusion of an antagonist of $\mathrm{H} 1$ histamine receptors reduces neurogenic vasodilatation (Akerman et al., 2002b). It therefore appears that mast cells make a contribution to neurogenic vasodilatation, but can be by-passed, as when CGRP is applied intraluminally (Bhatt et al., 2014). Besides histamine, the mast cell compound 5-hydoxytrypamine (5-HT, serotonin) also has a role (Keller and Marfurt, 1991; Terron and Martinez-Garcia, 2007; Wang et al., 2014).

5.2.2. Extravasation of plasma proteins from dural vessels. Changes in intracranial vascular endothelia in at least one location (parenchyma, leptomeninx, dura), that allow extravasation of proteins, are a common feature of brain pathologies and meningitis and are often associated with infiltration of intracranial tissues by immune cells. Stimulation of the trigeminal nerve or intravenous infusion of neuropeptides cause intracranial extravasation in the dura but not the parenchyma (Markowitz et al., 1987). Although this extravasation, per unit mass of tissue, is much smaller than in some extracranial tissues, such as lip and eyelid, extravasation in the dura can be conspicuous in infectious meningitides and it may also have a role in headache (Section 5.2.3); it therefore merits consideration.

Majno and Karnovsky (1961) showed that extravasation induced by histamine occurs in the rat cremaster muscle through gaps between endothelial cells, and both there and in the calvarial dura the leakage is from post-capillary venules, and not capillaries (Majno et al., 1961 and Gabbiani et al., 1970). Over time, the leakage is fairly uniform along the vessel, but this appears to be the integrated result of localized leaks each lasting no more than a few minutes (Horan et al., 1986; Miller and Sims, 1986; Svensjo and Grega, 1986). Quantitative measurements of extravasation in rat dura were made by Markowitz et al. (1987) using intravenous injection of radioactive albumin followed by post-mortem extraction from the dural tissue adhering to the calvaria. In vivo imaging through the thinned skull has shown leakage of vascular markers (quantum dots, Evans Blue, or fluorescein-labeled dextran) in mice infected with lymphocytic choriomeningitis virus (LCMV; Kim et al., 2009; Shaw et al., 2015, their Supplementary Fig.2), or with trypanosomes (Coles et al., 2015). In both cases, the leaky vessels were probably dural rather than leptomeningeal because they were close to the skull and because the dura contains long post-capillary venules (Fricke et al., 2001). Shaw et al. (2015) show leaks lasting no more than about 1 min and Coles et al. (2015) show localized leaks lasting only about $4 \mathrm{~s}$. The dural leaks may allow abrupt extravasation of trypanosomes (Coles et al., 2015) and in LCMV appear to be part of the immune response, for which Kim et al. (2009) observed "a tight correlation" between locally synchronized meningeal extravasation of putative neutrophils and vascular leakage. 
5.2.3. A note on headaches. Apparently, the only sensation that can be produced by stimulation of meningeal sensory fibers is pain, which arises from a subpopulation of trigeminal fibers, mainly those with endings on dural vessels (Fay, 1935; Penfield, 1935; Ray and Wolff, 1940). Hence, it can be concluded that headache is the perception of activity in neurons of the trigeminal system. Apart from mechanical distortion, trigeminal nociceptors respond to inflammatory mediators, such as histamine, which is among the repertoire released by mast cells (Section 8.1; Strassman et al., 1996; Bove and Moskowitz, 1997; Dux et al., 2012). The number of studies on cranial meningitides caused by infection is very small, but the available data show increases in meningeal mast cells (Dimitriadou et al., 1997), neutrophils (Kim et al., 2009), and T lymphocytes (Coles et al., 2015; Shaw et al., 2015), and release of noxious mediators by dural fibroblasts exposed to LPS in vitro (Wei et al., 2014). Activation of one trigeminal nerve terminal in the dura leads to activation not only of that particular branch, but also its collaterals by the axon reflex. This can produce positive feedback: for example, neural release of CGRP causes vasodilatation, which will mechanically stimulate other terminals (Jansen et al., 1992; Williamson et al., 1997a; Akerman et al., 2002a). In addition, trigeminal nociceptors become sensitized (Strassman et al., 1996; Boyer et al., 2014). Hence, although the precise mechanisms are unknown, it is not surprising that infectious meningitides can initiate and sustain the headache that is a common, usually minor, symptom.

The complexity of migraine is illustrated by the very large number of very different drugs that have a significant prophylactic effect (Jackson et al., 2015) and which presumably act on different sites, including both the dura and the trigeminal ganglion, neither of which is protected by the BBB (Rodriguez-Peralta, 1957; Eftekhari et al., 2015). In the case of migraine with aura, it is now generally accepted (after decades of justified hesitation) that the perceptual disturbance (usually visual) that announces the attack is the result of cortical spreading depression (CSD; Leao and Morrison, 1945; Pietrobon and Moskowitz, 2014; Burstein et al., 2015). CSD is a regenerative disruption of neuronal activity that slowly progresses as a wave over the cortex (Leao, 1944b) and is accompanied by dilatation of meningeal vessels (Leao, 1944a). MRI studies on patients with migraine with aura have shown a suppression of sensory response in the cortex, hyperemia, and hyperoxygenation, all features of CSD as described by Leao (Cao et al., 1999; Hadjikhani et al., 2001). CSD in rats induces prolonged activity in the ipsilateral trigeminal ganglion and the trigeminal nucleus caudalis (Zhao and Levy, 2015) and beyond into the contralateral high-order trigeminal nociceptive pathway (Cui et al., 2015). Activation of trigeminal neurons by CSD causes extravasation from the MMA (Bolay et al., 2002). The route of communication from parenchyma to dura is not known, but might be by centripetal ('antidromic') impulse traffic along the trigeminal afferents to the dura.

Migraine headaches that arise with no apparent external trigger, and other types of primary headache, are more problematic (Edvinsson and Uddman, 2005; Russo, 2015). For some decades, attention focused on vasodilatation and regenerative activation of trigeminal terminals in the dura, but more recent work shows less activity in the dura than expected. Magnetic resonance angiography during spontaneous attacks has shown no dilatation of the MMA (Amin et al., 2013). This was also true when attacks were triggered by previous infusion of the vasodilator nitroglycerine (Schoonman et al., 2008), and only little vasodilatation $(9.2 \%)$ was found when attacks were triggered with CGRP (Asghar et al., 2011). We do, however, find one report of plasma protein extravasation (Knotkova and Pappagallo, 2007). A key discovery was that certain drugs effective against migraine have no action in the dura but do inhibit activation in the spinal trigeminal nucleus (Sixt et al., 2009), an example being naratriptan, which inhibits capsaicininduced release of CGRP from the brain stem, but not from the dura (Kageneck et al., 2014; see also Tringali et al., 2012). Current evidence suggests that migraine attacks arise from abnormal intracerebral activity (Bolay et al., 2002; Shevel, 2012; Pietrobon and Moskowitz, 2013;Akerman and Goadsby, 2015), so that trigeminal neurons are activated centrally, the sensation of pain still being referred to the appropriate sensory field. Centripetal ('antidromic') impulses to the dura will then cause peripheral inflammation, sensitization, and positive feedback (Bolay et al., 2002; Edvinsson and Uddman, 2005; Hansen and Ashina, 2014). 


\section{Extravascular transport of molecules within the cranium.}

Quantitatively, the major supply of molecules (such as glucose and oxygen) to the neural tissue of the brain and the removal of others (such as $\mathrm{CO}_{2}$ and most excess amyloid $\beta$ ) occurs across the blood-brain barrier formed by the capillary endothelium and astrocyte endfeet (Section 3.4.1). In addition, there is good evidence that neurobiologically important substances, including some vitamins and DNA precursors (Johansson, 2014; Spector and Johanson, 2014) and perhaps cytokines (Devorak et al., 2015) are supplied, not directly from blood, but from CSF, and, inversely, that molecules can move from brain into CSF before reaching the venous system (Section 6.3). Since accumulation in the brain of certain catabolites and peptides is associated with neurodegenerative diseases, such as those of Alzheimer, Parkinson and Huntington (Weller et al., 2009; Klohs et al., 2014; Kress et al., 2014), their elimination is of great clinical interest. Mechanisms that have been proposed include transport within the walls of arteries, and convection by a flow of CSF. In either case, the material passes through the leptomeninx so the question is very relevant to meningeal physiology and pathology.

The literature on transport of substances in the brain is extensive and marked by controversy (e.g., Koh et al., 2005;Johanson et al., 2008; Pollay, 2010; Oreskovic and Klarica, 2010, 2011; Carare et al., 2014; Jessen et al., 2015; Spector et al., 2015a; Spector et al., 2015b; TarasoffConway et al., 2015;Engelhardt et al., 2017). The reviews by Davson (1972) and Hladky and Barrand (2014) take pains to explain the basic principles of fluid movement, and the latter raises a large number of open questions. In this review, we focus on the origin and fate of the CSF present in the meninges (Section 6.1), and the trafficking of molecules between the superficial cortex and the overlying meninges (Sections 6.2, 6.3).

\subsection{The 'macrocirculation' of CSF from the ventricles to exit from the cranium.}

Despite repeated questioning, the summary by Cushing (1925) appears to be supported by most evidence: much, probably most, CSF is secreted by the choroid plexuses in the ventricles and flows from there to subarachnoid cisterns and thence through channels on the surface of the brain, from where efflux routes return it to blood (Fig. 7A). In Sections 6.1.1 and 6.1.3 we will also mention evidence that this picture may be incomplete, as reviewed in detail by Hladky \& Barrand (2014) and Spector et al. (2015a,b).

6.1.1. Secretion of CSF by the choroid plexuses. The area of the secretory membranes of the choroid plexuses in rat increases with age and, at 30 days, reaches $75 \mathrm{~cm}^{2}$, which is almost half the total area of the brain capillary blood-brain barrier (Keep and Jones, 1990). Although additional sources have been suggested (such as the ventricle linings: Pollay and Curl, 1967), the 1.5 - 3 $\mu \mathrm{L} / \mathrm{min}$ of CSF produced in the ventricles of the adult rat comes essentially from the choroid plexuses (Mann et al., 1978; Preston, 2001; Han et al., 2009; reviewed in Spector et al., 2015a,b). The choroid plexus secretes into CSF low concentrations of neurobiologically active ions and molecules including vitamins (especially vitamin C), DNA precursors, hormones and cytokines which are necessary for brain development and homeostasis (Spector, 2009; Spector and Johanson, 2014;Devorak et al., 2015; Spector et al., 2015a). The rate of CSF production affects the delivery of these to the brain, and also intracranial pressure (Oshio et al., 2005). For these reasons, pharmacological modification of choroid plexus secretion is of clinical interest (Spina, 1899; Mott, 1910; Li et al., 2000; Johanson et al., 2008). In the reverse direction, the choroid plexus is affected by molecules in the CSF, such as interferon 1, that are produced in brain neural tissue (Baruch et al., 2014). Thus, flow within the ventricles is not purely unidirectional away from the choroid plexuses. Perturbation of CSF flow, as in hydrocephalus, causes alterations in CSF composition, the concentration of protein in the ventricles (relative to that in lumbar CSF) tending to be higher (sic) in patients with greater CSF movements (Puy et al., 2016).

6.1.2. Flow of CSF from ventricles to the surface of the cortex. CSF can flow from the lateral ventricles to the third ventricle and then through the aqueduct of Sylvius (or 'cerebral aqueduct') to the fourth ventricle. In Murinae, the only obvious conduits from there to the SAS are the foramina of Luschka ('lateral apertures') leading to the pontocerebellar cistern (Cammermeyer, 1971; Oda 
and Nakanishi, 1987; Fig. 7A). (In humans, there is an additional channel, the foramen of Magendie ('median aperture'), which connects the fourth ventricle with the cisterna magna.) In rats, there appears to be a net flow of CSF from the ventricles, caudad through the aqueduct of Sylvius, then to the SAS. Evidence includes that of Ghersi-Egea et al. (1996), who showed, in halothaneanesthetized rats, that sucrose injected in a lateral ventricle is carried to other ventricles and to subarachnoid cisternae with a rapidity indicative of bulk flow of CSF. Similarly, Fisher et al. (1983) found that calcitonin gene related peptide (CGRP) injected intraventricularly caused release of norepinephrine (presumably from the meninges) within minutes. Measurements using fluorescent tracers in mice showed no net flow in the reverse direction (from cisterna magna to ventricles; Iliff et al. 2013b; Iliff et al., 2013b; Bedussi et al., 2015).

Any net flux of CSF is minor compared to the to-and-fro movements that had already been observed in the second century AD by Galen (below the open atlanto-occipital membranes of some animals - see Du Boulay, 1972). Phase-contrast magnetic resonance imaging (PC-MRI) can (in animals with large brains) measure the changing velocity of CSF in the larger conduits, and also the movement of brain tissue, completely non-invasively (see Dreha-Kulaczewski et al., 2017, and Yildiz et al. 2017, for techniques). The oscillation of the velocity (typically measured in the aqueduct of Sylvius from the third to the fourth ventricle - Fig. 7A) has two main components, a one synchronized with the cardiac cycle (Bergstrand et al., 1985; Barkhof et al., 1994; Schroth and Klose, 1992a; Knobloch et al., 2014), and another synchronized with respiration (Schroth and Klose, 1992b; Dreha-Kulaczewski et al., 2015, 2017; Yildiz et al., 2017). In routine clinical PCMRI of CSF velocity, the imaging is synchronized to the cardiac cycle and the instantaneous velocity, caudad during systole and rostrad during diastole, is plotted as a function of time: this gives useful diagnostic information on brain pathologies, particularly hydrocephalus (e.g., Nitz et al., 1992; Scollato et al., 2008; Penn et al., 2011; Puy et al., 2016) but not cognitive impairment (Luetmer et al., 2002). Net flux, if it exists, is much smaller than the to-and-fro fluxes and its calculation requires accurate determination of a zero reference velocity (Hladky and Barrand, 2014). The relatively few groups that have published estimates of net flow in adult humans give widely varying mean values, but are unanimous that the flow is caudad (Feinberg \& Mark, 1987; Enzmann and Pelc, 1993; Gideon et al., 1994; Kim et al., 1999; Huang et al., 2004; Baledent et al., 2004; Piechnik et al., 2008; Yoshida et al., 2009; Penn et al., 2011; Bateman and Brown, 2012). The same techniques applied in cases of normal pressure hydrocephalus find net flow in the opposite (rostrad) direction (Kim et al., 1999; Bateman and Brown, 2012; Ringstad et al., 2016), with one exception (Gideon et al., 1994). In most cases, the authors point out that their results agree with results obtained by invasive, or indirect, classical methods. The contribution of respiratory movements to CSF flow has recently been re-examined using more powerful, real-time, PC-MRI (Chen et al 2015). There is agreement that inspiration causes an upward movement of CSF (from the fourth ventricle towards the third) but, confusingly, while Dreha-Kulakewski et al. 2017 find that this movement is greater than that caused by cardiac pulsations, Yildiz et al., 2017, find that it is smaller. The above three papers appear not to rule out agreement with the other PCMRI studies cited above, which found a net water flux superimposed on the to-and-fro movement.

Taken together, the PC-MRI results show that changes in pressure caused by blood flow or respiration can move CSF to-and-fro through the aqueduct of Sylvius, and supply some support for a normal net caudad flow, but confirm that, at least in pathological states, there can be sources of CSF in addition to the choroid plexuses.

In conscious rats, the mean CSF pressure in the cisterna magna appears to be higher than in the lateral ventricles (Verlooy et al., 1990; Sanchez-Valverde et al., 1995), which implies a pumping action in the cerebral aqueducts. Since both heartbeat and respiration produce large movements of CSF, these are candidate drivers of the net flow and, indeed, reducing cardiac pulsation has been shown to reduce movement of marker molecules through the brain (Rennels et al., 1985; Hadaczek et al., 2006; Section 6.2). However, in patients with normal pressure hydrocephalus, cardiac-driven to-and-fro movement of CSF can be maintained while net flow reverses (Kim et al., 1999; Bateman and Brown, 2012; Ringstad et al., 2016). Another possible 
driver of net flow might be the motile cilia that line the aqueducts and whose function appears to be necessary for the prevention of hydrocephalus (Ibanez-Tallon et al., 2004; Kousi and Katsanis, 2016). Their movement requires metabolic energy and, together with pulsation of the tissue, would be impaired in experiments in which blood flow is reduced. Hence, although the relative contributions to net CSF flow of heart beat, inspiration and ependymal cilia are uncertain, there is good evidence that the cilia play a role.

When the CSF system is perturbed, it becomes apparent that it is plastic and that the choroid plexuses are not the obligatory source of CSF. Strikingly, Milhorat et al. (1971) found that three months after their excision (in monkeys) CSF production was reduced by only one third; and in normal pressure hydrocephalus, CSF flows towards, rather than away from, the major sites of the choroid plexuses (Milhorat, 1975; Kim et al., 1999; Penn et al., 2011; Ringstad et al., 2016).

From the cisterna magna, it has been shown, by injection of particles (as in Indian ink), or high molecular weight dye, that CSF flows through the subarachnoid cisternae that surround the circle of Willis, and the paravascular spaces of the arteries that leave it to course over the hemispheres (Key \& Retsius, 1875; Kida et al., 1993a; Iliff et al., 2013a; Figs. 2CD, 6D, 7A). There appears to be no evidence that CSF flows forward from the cisterna magna through a continuous subarachnoid space over the cortices (Weed, 1914a, 1923; Kida et al., 1993a; Iliff et al., 2013a; Coles et al., 2015).

A

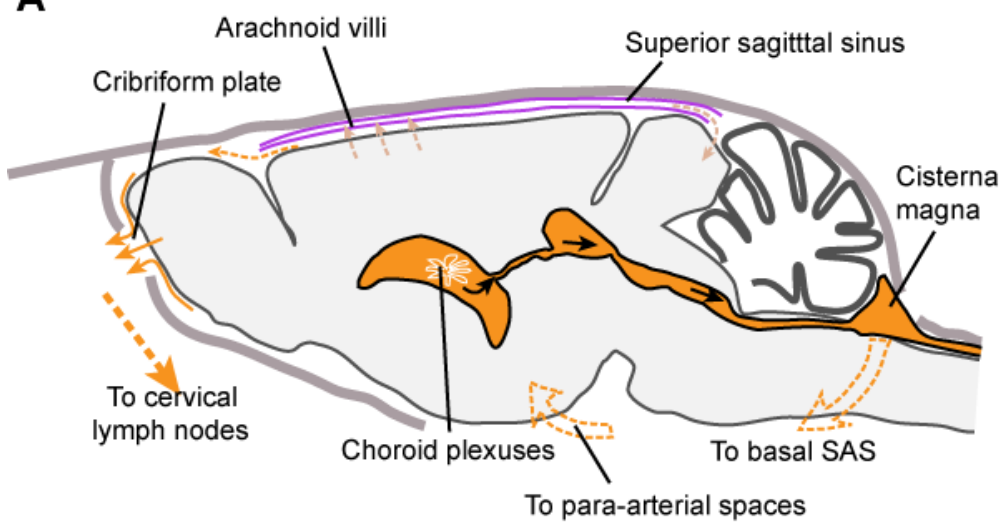

B

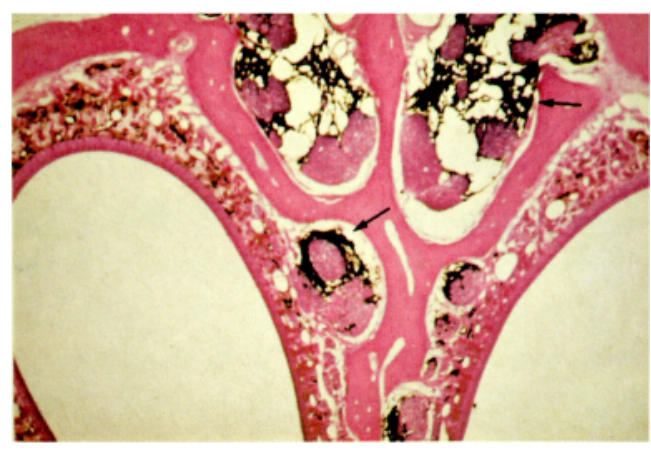

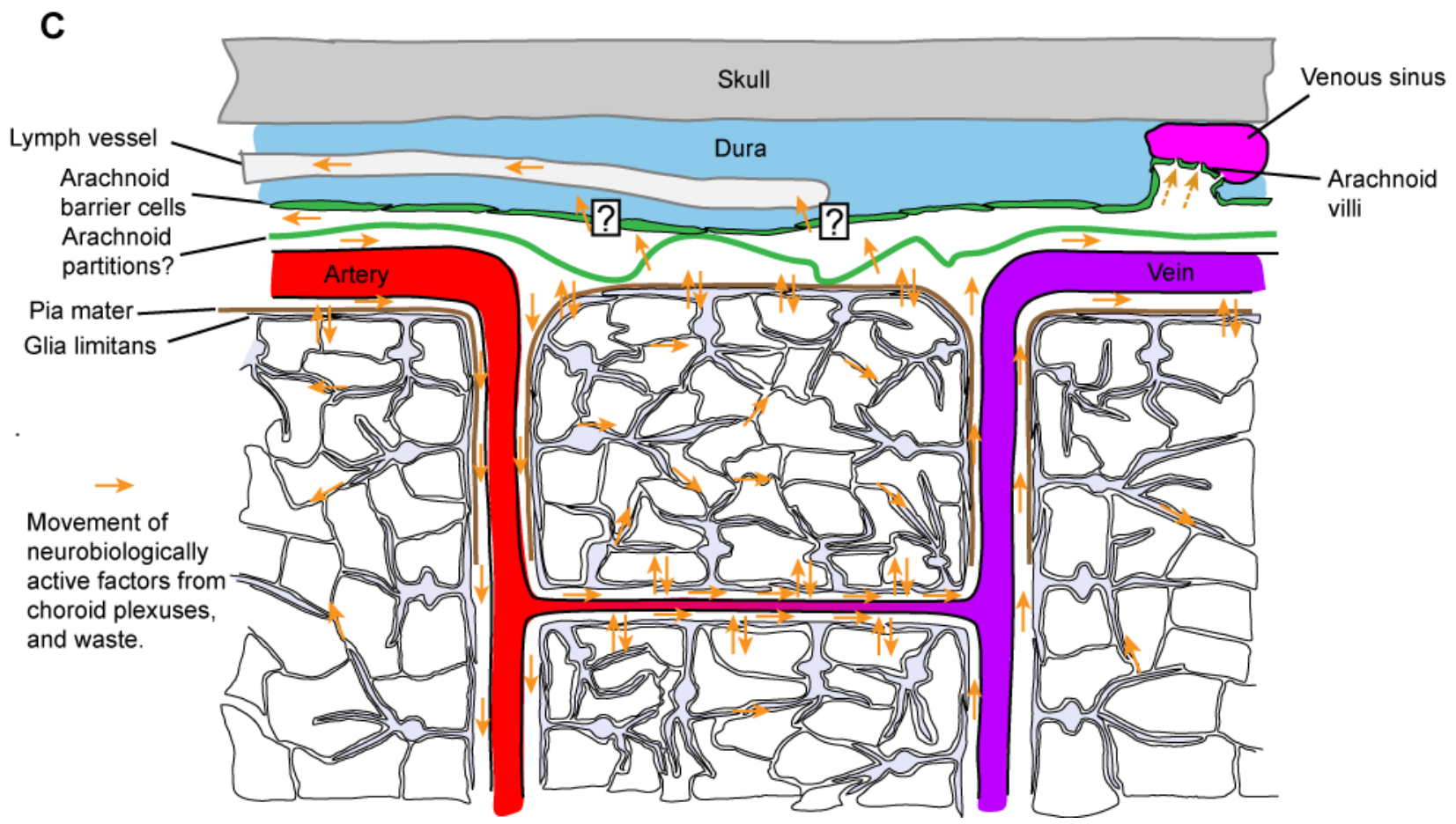


Figure 7. Conduits of CSF and ISF. A. The 'classical' sources and sinks of CSF. CSF excreted by choroid plexuses in the ventricles reaches the surface of the brain at the cisterna magna, flows through para-arterial spaces to reach leptomeningeal spaces and leaves the cranium to reach the cervical lymph nodes (the cribriform plate being a major route). CSF may also flow into veins via arachnoid granulations and the superior sagittal sinus. B. Ink injected in the cisterna magna of a rabbit fills orifices of the cribriform plate (arrows). By courtesy of H.Yamazumi (1989). C. Pathways of CSF flow in the cortex and meninges as hypothesized in Section 6.3. Molecules carried by CSF (orange arrows) follow paravascular routes along arteries, capillaries and veins. Solutes exchange with interstitium by diffusion, mainly to and from the pericapillary space. CSF may exit the cranium via subarachnoid spaces, via lymph vessels in the dura, or via arachnoid granulations into venous sinuses. Peptides can also be transported towards the pia within the walls of arteries (not shown).

6.1.3. Efflux of CSF from the cranium. CSF does not leave the brain across the capillary endothelium (see Hladky and Barrand, 2014) but by two main routes: into the venous sinuses of the dura; and to cervical lymph nodes via the nasal submucosa (Weed, 1914b; Pollay, 2010; Fig. 7A). Concerning the latter route, there has long been evidence that CSF passes into the deep cervical lymph nodes in large mammals (His, 1865; Schwalbe, 1869; Quincke, 1872), egress from the cranium being mainly through the cribriform plate (Key and Retzius, 1875; Weed, 1914b). The passage through the cribriform plate is along extensions of the SAS (Yamazumi, 1989; Kida et al., 1993a; Fig. 7B), and perhaps within lymph vessels, which are present in the dura just internal to the cribriform plate (Zwillinger, 1912; Furukawa et al., 2008) and, according to Aspelund et al. (2015), "some vessels pass[ed] through the skull into the nasal mucosa". Ligation of cervical lymph ducts increases CSF pressure (Földi et al., 1963) and causes accumulation of CSF in the lymph nodes that is reduced in knock-out mice lacking dural lymph vessels (Aspelund et al., 2015). By use of radioactive tracers, it has been shown that efflux to the lymphatics is prominent in rabbits (Bradbury et al., 1981; Bradbury and Westrop, 1983) and rats (Cserr et al., 1981; Krisch et al., 1983; Widner et al., 1987). More recently, the pathway has been shown with fluorescent markers in mice (Bedussi et al., 2015).

An anatomical substrate of the other main route of CSF efflux, into the venous sinuses of the dura, was described in man by Pacchioni in 1705 (cit. Brunori et al., 1993), who observed prominent granular extensions of the arachnoid penetrating the lacunae of the sagittal sinus (Fig.7A). In dogs, the granulations take a smaller form, the 'arachnoid villi', which can be stained by labeled CSF (Figs 1-5 in Weed, 1914c).

Arachnoid villi (from monkey) act as one-way valves whose resistance to flow from SAS to venous blood decreases sharply when the pressure differential is sufficient (Welch and Friedman, 1960). Concerning Murinae, arachnoid villi are rare in rats (Kida et al., 1993a), but Butler et al. (1975) found that high CSF pressure induced "transcellular channels within the endothelial cell cytoplasm" of the superior sagittal sinus.

Although there is also efflux of CSF into the spinal meninges and along the sheaths of other nerves, including the optic nerve and those passing through the jugular foramen (Pollay, 2010), debate has focused on the relative fluxes through the two major pathways: to the cervical lymphatics, and to the venous sinuses. Weed $(1914 \mathrm{a}, \mathrm{b})$, who strongly favored efflux to the venous sinuses, derided the opposite view of Cathelin (1903), and Weed's view was widely accepted (e.g., by Davson et al., 1970). However, when fluxes through the two pathways were compared directly (in sheep) it was found that the major route of efflux at normal CSF pressures was to the lymphatics, and only at high pressures did efflux to the venous sinuses predominate (Zakharov et al., 2004). This result, which could be predicted qualitatively from the valve function of arachnoid villi (Welch and Friedman, 1960), might explain the earlier emphasis on the venous pathway, since CSF marker solutions were usually injected under pressure. Concerning Murinae, Boulton et al. (1999) measured the appearance of radioactive albumin in blood and various organs $6 \mathrm{~h}$ after intraventricular injection, and also looked at the effect of obstructing cervical lymphatic drainage on the arrival of tracer in blood. They concluded that about half of the normal CSF efflux is to the cervical lymphatics. Unlike monkey and other large mammals, the resistance of the pathway into the venous sinuses of rat remains constant as CSF pressure increases (Jones and Gratton, 1989) - or 
at least remains high (Mann et al., 1978) - and does not drop markedly when the pressure exceeds a threshold value. A reason why venous efflux mechanisms (arachnoid villi or granulations) appear to be both more present and more effective in large mammals, compared to small ones (including neonates) may be that the pressures produced by mechanical shock are much greater for large brains, and evolutionary selection led to the elaboration of safety valves.

Many laboratories have measured CSF pressures in freely-moving rats and have reported values which vary widely, from rat to rat and from hour to hour, in the range $0-16 \mathrm{mmHg}$ with an overall average of $6.9 \mathrm{mmHg}$ (from ten articles, including Mandell and Zimmermann, 1980; Jiang and Tyssebotn, 1997; Guild et al., 2015). Hence it is likely that in Murinae, repartition of CSF efflux between the lymphatic and venous pathways is far from constant.

\subsection{Long-distance transport of molecules in the dorsal cortex.}

Although movement of molecules through the interstitium of neural tissue is well accounted for by diffusion (Syková and Nicholson, 2008), on a scale large enough to include blood vessels, much faster transport is observed, and marker molecules are found associated with blood vessels (Cserr and Ostrach, 1974; Cserr et al., 1977; Rennels et al., 1985; Zhang et al., 1992; Carare et al., 2008; Iliff et al. 2012). A debate about the existence and nature of paravascular transport as a link between the neural interstitium, CSF, and blood has continued from the time of His (1865) to the present day (Carare, et al., 2014; Brinker et al., 2014; Jessen et al., 2015; Hladky and Barrand, 2014; TarasoffConway et al., 2015; Spector et al., 2015b). We consider first whether there is net flow of CSF along paravascular space, and then (Section 6.2.2) whether or not this plays a part in the delivery of molecules to the neural brain and the removal of others.

6.2.1. Is there net flow of CSF along paravascular spaces? Spina (1899) and Weed (1914c) suggested that the clear liquid that exudes from discrete points on the exposed cortical surface is CSF that has emerged from paravascular spaces. Szentistványi et al. (1984) concluded that: "ISF is formed from plasma at the capillary wall, flows through intercellular clefts of the neuropil, and then drains from the nervous tissue via a network of low-resistance extracellular channels (including perivascular spaces) into cerebrospinal fluid (CSF) within the subarachnoid space". The evidence now, reviewed by Spector (2015b), is that this theory may account for the homeostasis of ISF, but not for any major contribution to the formation of CSF. Briefly, less than $10 \%$ of CSF originates in extrachoroidal sources in rat (Davson and Segal, 1996), the transporters necessary for the formation of CSF are sparse on brain capillaries (Mokgokong et al., 2014), and water exchange across brain capillary endothelium is limited, particularly in the cortex (Seo et al., 2002), as might be predicted from the lack of aquaporin (Wen et al., 1999; Iliff et al., 2012; but cf. Kobayashi et al., 2001). Further, several groups have shown transport along cortical paravascular space that is not upwards to the leptomeninx, but downwards. Wagner et al. (1974) and Lochhead et al. (2015) on rat, Klatzo et al. (1964) and Rennels et al. (1985) on cat, and Bedussi et al. (2015) on mouse, all found solutes arriving in the PVS after their introduction in the leptomeninx. Rennels et al. (1985) found differences between the different classes of vessels, with peri-arteriolar space being labeled before perivenular space. Iliff et al. (2012), using intravital two-photon microscopy, were able to distinguish arterioles from venules in vivo, either by tracing their connections to the vessels of the pia, or by expression of a marker of arterial muscle (NG2) in reporter mice, and they confirmed that fluorescent markers moved, within minutes, from PVS of pial arteries down the PVS of arterioles penetrating the mouse cortex (Figs 2F,G). The integrity of the dura appeared to be necessary for the paravascular transport, in agreement with earlier experiments by Ichimura et al. (1991), who had removed the dura (Iliff et al., 2012). Also in agreement with Iliff et al. (2012), Bedussi et al. (2015) found that, after infusion of $500 \mathrm{kD}$ fluorescent dextran in the cisterna magna, labeling "followed paravascular spaces along arteries into the parenchyma". In contrast, Arbel-Ornath et al. (2013) concluded that paravascular flow was in the opposite direction: "We found that the ISF drains [towards the pia] along perivascular spaces of arteries and capillaries but not veins." However, all but one of the images they show are of large horizontal vessels with adjacent spaces, as seen in their Fig.6, A-F, and these must be in the leptomeninx rather than the parenchyma. The exception is their Fig. $2 b(2)$, which shows a large penetrating artery with the marker dye labeling basement 
membrane and (in our opinion) perivascular space. They do not exclude the possibility that the dye arrived in this location from the pial para-arterial spaces.

The weight of old and new evidence favors transport down para-arteriolar space, but at least two mechanisms are possible. As confirmed by real-time measurements of their diameters by linescanning two-photon microscopy, arterioles in the superficial cortex pulsate (Iliff et al., 2013b), and when pulsation is reduced, movement of molecules through the cortex is slowed (Rennels et al., 1985; Hadaczek et al., 2006; Iliff et al., 2013). Pulsation will produce to-and-fro movement of perivascular fluid and mixing, which will result in faster transport than diffusion alone (Hladky and Barrand, 2014; Asgari et al., 2016). There is some evidence, also, for net bulk flow. Iliff et al. (2012) observed that $2000 \mathrm{kDa}$ dextran injected in the cisterna magna descended para-arteriolar space to reach an imaging plane $100 \mu \mathrm{m}$ below the pia. When the experiment was repeated in mice lacking aquaporin 4 in the astrocyte endfeet the arrival of the dextran in the para-arteriolar space of the imaging plane was delayed by about $5 \mathrm{~min}$, which would happen if bulk flow were slowed. (This is our reading of Fig. 4H in Iliff et al., 2012; the authors themselves say there was no change in build up of the dextran concentration.) Fig. S6 E shows that the para-arteriolar space was still patent in the absence of aquaporin, and Fig. S6 D shows that $40 \mathrm{kDa}$ dextran could still enter this space. Since aquaporin is a water channel, its absence might well cause reductions in water flow, from which would follow that there is normally net water flow down the para-arteriolar space. On the basis of mathematical models, Bilston et al., 2003 and Wang and Olbricht, 2011, suggested that net paravascular flow (parallel to the blood flow) could be produced by the waves of vessel dilatation caused by the heart beat. However, as pointed out by Asgari et al., 2016, the first of these models uses unrealistically low values for the wavelength of the dilatation, while the second, an analytical model, gives results independent of the wavelength. From their own model, Asgari et al., 2016, concluded that the main mechanism of net solute movement along paravascular space is diffusion, with dispersion by mixing making a contribution of $10-30 \%$, and net flow of water even less. This theoretical prediction does not account for the rapid transport observed experimentally by Rennels et al., 1985, and Iliff et al., 2012. A possible factor, ignored by Asgari et al., 2016, is a static pressure gradient from para-arteriolar space to paravenular space.

Iliff et al., 2012, also found that in tissues fixed at longer times $(>1 \mathrm{~h})$ after injection in the cisterna magna, tracers were present along capillaries and venules. On the basis of these and other results, Iliff, Nedergaard and colleagues proposed the 'glymphatic' theory (Jessen et al., 2015). Concerning the dorsal cortex, this theory states that CSF flows down para-arteriolar space, water and solutes pass into interstitium, and water and solutes leave interstitium into paravenular space where they create an upward flow of CSF (Iliff et al., 2012; Jessen at al., 2015). To account for the motive force required for this circuit, the authors "speculate that the transglial water movement, presumably driven by the hydrostatic pressure of para-arterial bulk flow, drives solute flux from the paravascular space into the interstitium" (Iliff et al., 2012). A feature of the glymphatic theory is that there is no net secretion of CSF into SAS, and it in no meaningful way conflicts with the conclusion that the choroid plexuses are the principal sources of CSF. The finding that markers injected in midbrain appear in CSF in the cisterna magna (Szentistvanyi et al., 1984) appears to be compatible with both the glymphatic theory and the net source of CSF being the choroid plexuses.

The glymphatic theory can be considered at three levels: the experiments and their immediate interpretation; the conclusion that there is a glymphatic circuit in which CSF flows down para-arterial space and up paravenous space; and speculation as to the physical mechanisms. The speculation that there is bulk flow from PVS into and through interstitium driven by the "hydrostatic pressure of para-arterial bulk flow" has been questioned by at least three laboratories (Hladky and Barrand, 2014; Spector et al., 2015b; Smith et al., 2015) and is not supported by a mathematical model (Jin et al., 2016). These objections on theoretical grounds cannot be imperative, but they do encourage scrutiny of the experimental results and their interpretation.

6.2.2. A critique of the glymphatic circuit. Iliff et al. (2012) concluded that glymphatic efflux was greatest into the medial internal cerebral veins and the lateral-ventral caudal rhinal veins, but in this 
review we continue to focus on the dorsal cortex. As described above for $2000 \mathrm{kDa}$ dextran, Iliff et al. (2012) examined transport of molecules from CSF into the brain after injecting $10 \mu \mathrm{L}$ of fluorescent marker solution into the cisterna magna over 5 min. In intravital imaging of an XY plane in the somatosensory cortex, $100 \mu \mathrm{m}$ below the pia (their Fig. S3C), the mean of the concentrations of $3 \mathrm{kDa}$ dextran in the para-arteriolar spaces was seen to increase at a steady rate from time zero to $25 \mathrm{~min}$. We note in passing that this is weak evidence against the net flow described above as it tends to suggest transport by mixing rather than net flow: with net flow, the arrival of the bolus of dye might appear as a step change in concentration (more-or-less smeared out by mixing). In the tissue surrounding arterioles the concentration also increases linearly from time zero and, strikingly, the concentration in tissue surrounding venules is at all times indistinguishable from that near arterioles: the supposed transport from near arterioles to near venules occurs in the absence of a detectable concentration gradient (Fig.S3C). In the authors' words: "The lack of a statistically-significant difference between the curves indicates that this tracer enjoys relatively unrestricted movement outward from the para-arterial space" (Iliff et al., 2012). Thorne et al (2006) measured the diffusion of $3 \mathrm{kDa}$ dextran from an instantaneous point source in the exposed somatosensory cortex of rat. After $1 \mathrm{~min}$, the concentration $100 \mu \mathrm{m}$ away was, very approximately, half that at the origin, illustrating how net transport by diffusion requires a concentration gradient. Net transport in the absence of a concentration gradient could, in principle, occur by convective flow, as suggested by Iliff et al. (2012), although the dye would not reach the venules immediately. Jin et al (2016) have modeled the effects of both diffusion and convection. They calculate that diffusion alone could in principle maintain the dye flux through the interstitium, but this would require concentration gradients in the XY plane, contrary to the quantitative results of Iliff et al., 2012, Fig.S3C. On the other hand, convection is unlikely because it would require a high hydrostatic pressure difference between arterioles and venules (Smith et al., 2015; Jin et al., 2016), and the arrival of dye at the venules would not be instantaneous.

Figs 1L,M and S1 of Iliff et al., 2012, and Fig. 1A of Iliff et al., 2013b (which we reproduce as Fig. 2E) show coronal sections of tissue fixed after cisternal injection, and in these $3 \mathrm{kDa}$ dextran or ovalbumin (unlike $2000 \mathrm{kDa}$ dextran) is seen to label cortical tissue downwards from the pial surface, much as Roseberg et al. (1980) showed diffusion into gray matter from the ventricular ependyma. The glymphatic circuit might explain this by the removal of marker from the CSF as it descends the para-arteriolar spaces, so that there is less marker to label deeper tissue, but it seems also possible that $3 \mathrm{kDa}$ dextran moves directly through the pial surface, which was the interpretation of Dunker et al. (1976) of analogous experiments with a $44 \mathrm{kDa}$ protein. The pial surface is bounded by astrocyte endfeet expressing aquaporin (Frigeri et al., 1995; Nielsen et al., 1997) and Fig. 4E in Iliff et al., 2012 shows that $45 \mathrm{kDa}$ protein enters the superficial cortex more slowly in mice lacking aquaporin. Might diffusion from the pia account for the equal build-up of 3 $\mathrm{kDa}$ dextran near venules and arterioles $100 \mu \mathrm{m}$ below the surface in Fig. S3C? Comparing the images in Figs S3B,C with Figs $2 \mathrm{~B}$ and S3A, it is apparent that there was tissue movement $>40 \mu \mathrm{m}$ in the XY plane over $30 \mathrm{~min}$; given that a craniotomy had been made, one wonders if there was also movement in the $\mathrm{Z}$ direction that could also have affected the quantitative results?

From the coronal sections in Fig. 4E of Iliff et al., 2012, it appears that movement of $45 \mathrm{kDa}$ protein from the pial surface through the astrocyte endfoot layer and into the cortex is reduced in AQP4 null mice. Although the mechanism of this reduction in protein movement is not evident (Smith et al., 2015) it might be associated with a reduction in water flow. The endfeet facing the PVS of descending arteries have only low expression of aquaporin (Iliff et al., 2012, Fig. S7A), but, of course, a reduction of para-arteriolar flow in AQP4 null mice could result from an effect downstream. Our conclusions are that the results in Iliff et al., 2012 provide fair evidence for net flow of CSF down the PVS of cortical penetrating arterioles, but only weak evidence for movement of $3 \mathrm{kDa}$ dextran from para-arteriolar space into the interstitium in the superficial dorsal cortex. We suggest that the penetration of the interstitium in this location is mainly by diffusion from the pia mater, the flux being, for some reason, reduced in AQP4 null mice.

To study the distribution of markers deeper below the cortical surface and at longer times 
after intracisternal injection, Iliff et al. (2012) examined sections of fixed tissue. Their Fig 3A shows that $10 \mathrm{~min}$ after intracisternal injection, $45 \mathrm{kDa}$ protein can reach the basement membranes of parenchymal capillaries. At $3 \mathrm{~h}$, it is seen accumulated along venules as well as capillaries (their Fig. 3G,H), in qualitative agreement with Rennels et al. (1985). If we accept that the marker reached the capillary basement membrane from the peri-arteriolar space, then this is evidence of transport (diffusive, dispersive, or perhaps convective) along the capillary basement membrane towards the venules. Strikingly, direct, real-time, measurements of vessel diameter show that cortical venules pulsate as much as arterioles (Iliff et al., 2013b) so that transport would be aided by dispersion along the paravascular space of venules was well as of arterioles (Asgari et al. 2016). Between the capillary basement membrane and all parts of the brain interstitium, diffusion should be adequate for exchange of molecules (Ohata and Marmarou, 1992; Jin et al., 2016), and, indeed, Iliff et al. (2012, Fig. 1O) show that accumulation in the brain of mannitol (182 Da) or dextran (10 $\mathrm{kDa}$ ) injected intracisternally depends strongly on molecular weight, as expected for transport by diffusion. In summary, convective paravascular flow of CSF from arteries via the far more numerous capillaries to veins seems to account for many observations. Why this should be reduced in AQP4 null mice is not clear (Smith et al., 2015), but this knock-out is known to produce some remodeling of the tissue, as shown by the increase in the extracellular space of the neural tissue (Yao et al., 2008). The glymphatic theory of Iliff et al. (2012) differs from our paravascular circuit theory in that they propose that there is convective bulk flow from arterioles to venules.

\subsection{Clearance of exogenous and endogenous molecules from the cortex}

Over dimensions big enough to include blood vessels, molecules injected in brain tissue are cleared from the site of injection at rates little affected by molecular weight (Cserr et al., 1977, 1981) and reduced in AQP4 null mice (Iliff et al., 2012 Fig. 5A). These results show that clearance involves more than diffusion through the interstitial space (which is slower for larger molecules) and is perhaps affected by the properties of the astrocyte endfeet. Concerning the cortex, three major routes have been proposed: (1) Bulk flow towards the pia along para-arteriolar and paravenular spaces. (2) Bulk flow along the paravenular spaces. (3) Transport along the basement membranes of capillaries towards the arterioles and thence between the smooth muscle cells in the tunica media of the artery wall and so to the brain surface. Some arguments against the first route have been given in Section 6.2.1. In addition one can make the evolutionary argument that the neurobiologically active molecules secreted by the choroid plexuses (Spector and Johanson, 2014; Johansson, 2014) require some route of delivery to the neural tissue, i.e. that CSF from the choroid plexuses must be distributed into the parenchyma, as is indicated by tracer molecules injected in the cisterna magna (Iliff et al., 2012 Fig.1H,K).

6.3.1. Clearance by paravascular routes. Since there is evidence that CSF descends the paravascular space of arteries (Section 6.2.1), if there is clearance by bulk paravascular flow it must be along venules and veins. After intracisternal injection of small $(3 \mathrm{kDa})$ fluorescent molecules, Iliff et al. (2012, Fig. S3C) found a steady increase from time zero to $35 \mathrm{~min}$ in fluorescence from ascending veins $100 \mu \mathrm{m}$ below the cortical surface. This appears to be the only report of intravital imaging of extravascular markers associated with veins, and we suggest that contaminating signal from dye diffusing downwards from the pia has not been excluded (Section 6.2.1). At considerably longer times after injection into CSF, marker has been found associated with venules and veins (Rennels et al., 1985; Iliff et al., 2012; Rangroo-Thrane et al., 2013). These observations were made on tissue which had been fixed, a process which would have involved diffusion of marker during fixation (Dunker et al., 1976), and either its attachment to some substrate or its removal by washing. After this treatment, Rennels et al. (1985) identified a site of accumulation of protein marker as the basement membrane of venules, while Rangroo-Thrane et al. (2013, Fig.2f) found a lipophilic marker (tetramethylrhodamine) in the 'paravascular space' of venules. These results seem to be compatible with the possibility of clearance from the interstitium by bulk paravascular flow along venules, although, as Rennels et al. (1985) state "arterioles were more densely labeled than were ......venules or veins of comparable size, regardless of the period of HRP circulation". 
Observations after injection of markers into brain tissue give more confusing results. After injection of HRP (44 kDa protein) in the midbrain of rat, Szentistvanyi et al. (1984) found labeling of penetrating arteries ("between, rather than within, adventitial cells"). However, this was $2 \mathrm{~h}$ after the injection, and they show that, at earlier times, the HRP appeared in CSF in the cisterna magna and in paravascular channels of cerebral arteries: at $2 \mathrm{~h}$ the HRP was probably descending the arteries (see Section 6.2.1.) Carare et al. (2008) injected $3 \mathrm{kDa}$ dextran and $45 \mathrm{kDa}$ albumin in the mouse striatum and after fixing at $5 \mathrm{~min}$ found them colocalized with laminin in basement membranes of capillaries and in arteries in the basement membranes "between the smooth muscle cells in the tunica media of the artery wall", but "no tracer was seen in the walls of veins". At 30 min the markers were in basement membranes of arteries, but no longer of capillaries. From then on, both markers were also found within peri-arterial macrophages. Carare et al (2008) suggest that the markers were transported by arterial pulsation against the direction of blood flow, and mathematical models support this possibility (Schley et al., 2006; Coloma et al., 2016). Injections of $45 \mathrm{kDa}$ albumin in the striatum were also made by Iliff et al. (2012) who report different results. They show (in their Fig. S5) accumulation at 60 min around capillaries and venules, which were distinguished from arterioles by punctate, rather than continuous, expression of NG2. These images are not accompanied by images of arteries. Neither paper shows high magnification cross-sections of venules or penetrating veins. I.e., neither paper shows high magnification images to illustrate the absence of marker in the rejected pathway. Possible fixation artifacts need to be borne in mind: diffusion, cross-linking to substrate, and washing (Dunker et al., 1976), but both groups used the same initial method of fixation, namely perfusion with $4 \%$ paraformaldehyde. One observation that argues against pericapillary labeling as a fixation artifact is that although labeling of artery walls is persistent (Rennels et al., 1985; Carare et al., 2008) pericapillary labeling was found by Carare et al. (2008) to be transient. Further, by intravital imaging, Iliff et al. (2012 -Fig. 2H) showed that it is possible for $2000 \mathrm{kDa}$ dextran to diffuse from paravascular space into basement membrane in vivo, at least in the case of pial arteries.

Amyloid $\beta$ and other markers of brain disease pass from ISF not only across the BBB but also into the CSF circulation (Weller et al., 2008; Roberts et al., 2014). An extravascular route to the SAS associated with blood vessels is suggested by the findings that patients with Alzheimer's disease have parenchymnal angiopathy (see Weller et al., 2008), decreased flow of CSF (Golomb et al., 2000; Silverberg et al., 2003; Tarasoff-Conway et al., 2015) and increased stiffness of the arterial walls (Hughes et al., 2013(Hughes et al., 2014)). Amyloid $\beta$ is found in the walls of arteries (Wisniewski and Wegiel, 1994), and there is therefore a presumption that it is eliminated by transport towards the pia along arterial walls (Preston et al., 2003; Weller et al., 2008; Carrare et al. 2008; Carare et al, 2013; Tarasoff-Conway et al., 2015). However, given the remarkable distances over which injected markers can be dispersed (e.g., Szentistványi et al., 1984; Carare et al., 2008; Iliff et al., 2012) one can ask whether the continued presence of a molecule indicates rather that it was not in the process of being cleared.

The contradictory (or complementary?) observations of Carare et al. (2008) and Iliff et al. (2012) demand the adjudication of other experimenters, and this appears to be lacking. Bedussi et al. (2015), who did not perfuse with fixative, differ from both groups in finding that "small vessels close to the injection site did not show tracers". Lochhead et al. (2015) did not distinguish between arterial and venous vessels.

Rennels et al. (1985), Carare et al. (2008), Iliff et al. (2008) and Rangroo-Thrane (2013) all report the early arrival of ISF or CSF marker molecules in the basement membranes of capillaries. For molecules diffusing randomly through the neural interstitium, it is the sheath of the dense capillary bed that offers the nearest and largest area of escape. If there is ready diffusion between the capillary sheath and the neural interstitium, the extracellular concentrations should be the same, but the total concentration will be about five times greater in the capillary sheath because of the absence of cells, which occupy some $80 \%$ of the neural tissue (Syková \& Nicholson, 2008). In addition, possible shrinkage of pericapillary space during fixation would increase the concentration of marker and the fluorescence signal. Where does the marker go next? Iliff et al. (2012) imply 
that it is carried by bulk flow of CSF towards the venules; Carare et al. (2008) suggest that it moves towards the arterioles. On the time scales involved, transport of material by diffusion alone along a pericapillary space might be sufficient, as it is through the neural interstitium itself. [Indeed, transport by diffusion would be faster than through ECS because the volume is not reduced by cells and there is less tortuosity. If it were a true space, then, compared to the neural interstitium the quantity transported by diffusion would be $\lambda^{2} / \alpha \approx 13$ times more than in the interstitium, where $\lambda \approx 1.6$ is the tortuosity factor and $\alpha \approx 0.2$ is the volume fraction (Syková and Nicholson, 2008)].

In the absence of conclusive experimental results, we tentatively suggest the following hypothetical scheme. CSF flows from paravascular space of pial arteries down para-arteriolar space, along a narrow pericapillary space and up paravenular space, being driven by vascular pulsation. Solutes exchange with the interstitium by diffusion, mainly to and from the pericapillary space (Fig. 7C). In addition, as maintained by Carare et al. (2008, 2013, 2014, see also Engelhardt et al., 2017) molecules are transported up the walls of penetrating arteries. It is not obvious that the fluxes of glucose, oxygen, $\mathrm{CO}_{2}$ etc. that cross the capillary endothelium would interfere with this scheme.

\subsection{CSF Pathways through the cortical meninges.}

Where the leptomeninx includes the ventral cisternae or bridges the cortical sulci of primates, there is a cavernous SAS punctuated by trabeculae round which CSF appears to flow freely (Fig. 2C; Kida et al., 1993a). Over the convexities of the cortex there are membranous septa and occlusions which may direct the flow of CSF along different pathways (Zhang et al., 1992). Marker particles or molecules arriving in the cortical leptomeninx from the cisterna magna are initially confined to spaces on either side of the arteries (Fig. 6.A-F; Key and Retzius, 1875; Kida et al., 1993a; Iliff et al., 2012; Coles et al., 2015). The confinement seems to last longer if the tracer consists of particles such as Indian ink (Kida et al., 1993a) or molecules of high molecular weight, such as the $2000 \mathrm{kDa}$ dextran used by Iliff et al (2012; Fig. 6F), than if a lower molecular weight is used, such as the 625 Da Texas Red in Figs 6A-D, suggesting that the lateral movement is by diffusion. This observed segregation of para-arterial and paravenous CSF would be necessary if the glymphatic system were to clear waste efficiently. Beyond this, almost nothing is known beyond the complex compartmentation described by Krisch et al. (1983, 1984). There have been suggestions that CSF secreted by the choroid plexuses may be kept separate from that originating as ISF (rejecting the glymphatic theory) and directed respectively to arachnoid villi and the cribriform plate (Kida et al., 1993a). How the dural lymph vessels participate in the CSF circulation is unknown: Louveau et al. (2015) simply shown an arrow crossing the arachnoid membrane, which is thought to be impermeable (their ED Fig.10).

\section{Where are we? Identifying locations in the murine cortical meninges.}

Particularly in disease, it can be important to identify the tissue compartment (dura, leptomeninx or parenchyma) in which events are occurring, such as infiltration by pathogens or cells of the immune system, or extravasation. For the powerful techniques of transcranial intravital imaging and

characterization of cells by fluorescence activated cell sorting (FACS), precise identification of the compartment is rarely trivial. 

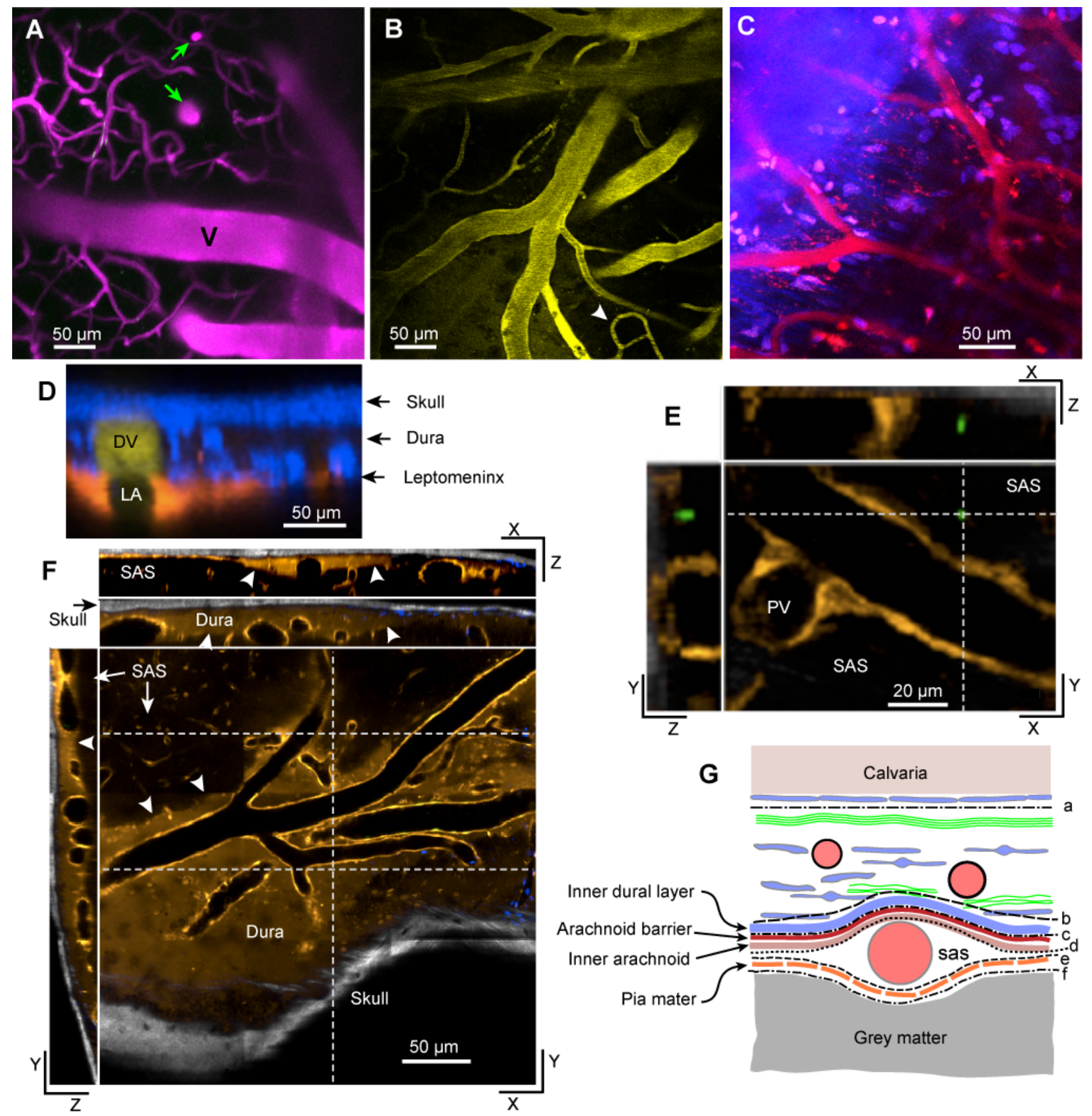

Fig.8. Identifying locations in the meninges. A-F. Vessels in and above the superficial left parietal cortex of mice, observed in vivo through the skull with a two-photon microscope.. A. A Z-projection, $244 \mu \mathrm{m}$ deep, including sinuous, horizontal, intraparenchymal capillaries, penetrating vessels, which appear as disks (green arrows), and veins (V) emerging into the meninges and flowing medially (right). B. An X-Y plane mainly showing dural vessels including small vessels with characteristic anastomoses (arrowhead). C. A shallow Z stack, $5 \mu \mathrm{m}$ deep, showing vessels just below the skull with the branching pattern characteristic of the dura. The image plane is slightly oblique, going from skull (blue, upper left) into the dura (lower right). Nuclei of some dural cells have been labeled blue by intravenous injection of furamidine (see Section 4.2.1). The blood plasma was labeled with quantum dots, and some appear to have been phagocytosed by extravascular cells. D. Vertical section showing a labeled dural vessel (DV) overlying an unlabeled leptomeningeal artery (LA) with paravascular spaces labeled in red by infusion in the cisterna magna. Dural nuclei were labeled by intravenous infusion of furamidine. (A-D) modified from Coles et al., 2015. E. Endothelial cells of vessels in a 'DsRed' mouse expressing DsRed under control of a $\beta$ actin promoter and cytomegalovirus enhancer cassette (Vintersten et al., 2004). The image shows an XY plane extracted from a Z stack and reconstructions of two orthogonal vertical planes, which pass through the dashed lines in the XY plane. The presence of both a horizontal and a vertical vessel (PV) indicates that the stack is in the pia. The green object is an extravascular Plasmodium berghei. Reproduced from Shaw et al., 2015. F. A superficial Z stack from a 'DsRed' mouse including skull bone. Orthogonal 
planes are shown. White arrowheads indicate the apparent boundary between leptomeninx and dura. By courtesy of P.J. Stewart-Hutchinson, with added labeling. G. Schematic showing possible planes of cleavage (a-f) in the preparation of wholemounts. See Section 7.2.

7.1. Meningeal landmarks for intravital imaging. Second harmonic generation (SHG) from skull bone readily defines the outer boundary of the dura (Figs 3D,4F, 8D) and extensive mats of collagen, also visible by SHG (Fig. 4B), are probably within the dura. So, too, are nuclei (other than those of vascular endothelium) labeled by intravenous injection of furamidine (Fig.3A,B, 4C). Blood plasma is readily labeled by intravenous injection, and visualization of blood vessels can help to identify the anatomical compartment under observation. The cortex is unambiguously identifiable by the characteristic sinuosity of its capillaries as they course parallel to the brain surface, and by the vertical arterioles and venules that they connect (Fig. 8A). All large horizontal vessels are in the meninges, most pial vessels being embedded in the brain surface and therefore in the same plane as capillaries in nearby parenchyma (Ichimori et al, 1991; Fig. 8A). The dura contains the meningeal arteries and their branches, and veins pass at the level of the dura to reach the SSS (Fig.2A). Capillaries and post-capillary venules are extensive in the dura and anastomoses are common (Fig.8B,C). Para-arterial spaces in the leptomeninx can be labeled by infusion in the cisterna magna (Fig. 6A-F), but this is laborious. 'DsRed' mice, in which DsRed is expressed under control of a $\beta$ actin promoter and a cytomegalovirus enhancer cassette (Vintersten et al., 2004) show fluorescence from vascular endothelial cells and, apparently, also from throughout the dura, but not the leptomeninx (Fig. 7E,F). Hence, in these mice, it appears to be possible to distinguish the dural and leptomeningeal compartments in vivo without injecting any marker. In fixed tissue, fibroblastlike cells, probably in the dura, can be labeled with antibodies to ER-TR7 (Kim et al., 2009).

\subsection{Mechanical separation of the dura, arachnoid and pia.}

In many studies using antibodies to identify types of immune cells in the meninges, layers are separated mechanically before or after fixation, and cells identified either in wholemounts or by FACS. The planes of cleavage, which determine whether dissected tissue is dura, arachnoid, pia, parenchyma, or some mixture, appear to depend on species, method of dissection, and tissue fixation.

As is well known to electrophysiologists (e.g. Greenhill et al., 2015), a window of calvarial bone can be excised in vivo in both rat and mouse while leaving in place the collagenous layers of the dura (Alexander, 1875; Masuda et al., 2007; Terron and Martinez-Garcia, 2007). However, if the unfixed brain is scooped carefully from the calvaria, meningeal tissue remains attached to the bone: Ottosson and Edvinsson, 1997; Rozniecki et al., 1999; Schwenger et al., 2007; Syed et al., 2012; Jansen-Olesen et al., 2014, (Schueler et al., 2014)for rat; Sayed et al., 2010; Walker-Caulfield et al., 2015, for mouse. This adhering tissue certainly includes the arteries and nerves of the dura, and excludes the major leptomeningeal vessels, as seen in wholemounts dissected from the calvaria, either fresh (Ottosson and Edvinsson, 1997; Syed et al., 2012), or after fixation (Rozniecki et al., 1999; Sayed et al., 2010). Historically, it was expected that cleavage of the meninges would occur between between the inner dural layer and the arachnoid barrier membrane ('c' in Fig. 8G), frequently described as the site of a virtual subdural space [see Haines (1991) for a list of textbook references]. The best evidence for possible cleavage along this interface appears to be the electron microscopy of Andres (1967, on dog) who sometimes observed a subdural space, but which he considered to be an artifact of fixation. Other electron microscopists do not report a subdural space, although they often show spaces within the dura (Haines, 1991), and Frederickson (1991), who set out to answer the question, found that, in fresh or fixed meningeal tissue of guinea pig, cleavage occurred within the inner layers of the dura ('b' not 'c' in Fig. 8G). However, after removing the fresh mouse brain from the calvaria and fixing and sectioning it, Sayed et al. (2010) observed sections with only the pia attached (cleavage at 'e') and were able to dissect 'pial wholemounts'. Recent publications accept the possibility that on removal of the brain, part, at least, of the arachnoid can remain attached to the mouse calvaria, and refer to 'calvarial meninges' (Christy et 
al., 2013; Walker-Caulfield et al., 2015) or 'dura/arachnoid' (Louveau et al., 2015).

If the cranial tissues are first fixed by perfusion before the calvaria is removed, a difference is described between rats and mice. In rats, the dura tends to stay not with the bone but with the brain, from which it can be removed as a dural wholemount (Messlinger et al., 1993; McMenamin, 1999). It is possible that, in this case, the lower plane of cleavage is within the arachnoid ('d' in Fig. 8G; McMenamin, 1999). In contrast, in the mouse, perfusion fixing appears not to prevent the dura from remaining with the calvaria (Arac et al., 2014).

\section{Immune cells in the brain and meninges in health and disease.}

The healthy neural brain (i.e., not including the meninges, choroid plexuses, ventricle walls or perivascular sheaths) contains very few immune cells apart from microglia (Section 8.4). Early experiments, notably by Medawar (1948), showed that foreign tissue grafted in the brain can survive, whereas similar tissue grafted in extracerebral sites is destroyed by the host's immune system. Despite this phenomenon, the neural brain is far from being immunologically isolated. It can 'mount robust immune responses to both infections and sterile injuries' (Russo and McGavern, 2015), and is the site of autoimmune pathologies, notably multiple sclerosis. The rodent model of this, experimental autoimmune encephalitis (EAE), can be induced by subcutaneous injection of an antigen found within the brain, such as myelin oligodendrocyte glycoprotein basic protein, together with adjuvent (e.g., Kuerten et al., 2008). Immune cells then gain access to CSF and the parenchyma through the choroid plexus (Engelhardt et al., 2001) or the meninges (Ransohoff et al., 2003; Bartolomäus et al., 2009). The afferent arm of the immune response is also effective, and $\mathrm{T}$ cell-dependent antigens yield higher serum antibody titers when introduced into brain tissue than into conventional extracerebral sites (Gordon et al., 1992). These immunological communications between the neural brain and the extracranial immune system pass through the perivascular spaces and the meninges, which are sites of immune cell residence and can be sites of intense inflammation. Current theories of the full process, from antigen presentation to recruitment of immune cells to the parenchyma, are reviewed elsewhere (Galea et al., 2007; Ransohoff and Engelhardt, 2012; Takeshita and Ransohoff, 2012; Carare et al., 2014; Russo and McGavern, 2015).

Resident immune cells that can be imaged through the skull are found in five main sites: within blood vessels, extravascularly in the dura and the leptomeninx, apposed to the abluminal surface of penetrating cerebral vessels, and in the superficial parenchyma. Cells of the classical immune system can participate in normal brain function, as in the case of $\mathrm{T}$ cells, which accumulate in the meninges during cognitive tasks (Derecki et al., 2010) and contribute to the neurogenesis necessary to avoid dementia (Price et al., 1988; Wolf et al., 2009; see Anandasabapathy et al., 2011 for further references). Conversely, the immune response to bacterial lipopolysaccharide (LPS) includes 'non-immune' cells. When LPS is applied to dural fibroblasts, inflammatory factors including IL-6 are released (Wei et al., 2014) and when injected intraperitoneally, the changes in the transcriptome of endothelial cells of the blood-brain barrier suggest that they secrete prostaglandin $\mathrm{E}_{2}$ (Vasilache et al., 2015).

Much of what is known of CNS immunology has been learnt from studies of EAE (Constantinescu et al., 2011; Brendecke and Prinz, 2015; Ransohoff et al., 2015). Induction of EAE activates dural mast cells within $24 \mathrm{~h}$ and this is followed by accumulation in the dura of neutrophils, macrophages, dendritic cells and T cells (Christy et al., 2013; Walker-Caulfield et al., 2015). T cells are observed to pass from the leptomeninx along the perivascular spaces of penetrating arterioles of the CNS (Bartolomäus et al., 2009; Walker-Caulfield et al., 2015) and immune cells of several types are later found in the cerebrum (Walker-Caulfield et al., 2015).

Apart from EAE, other sterile pathologies causing menigitis include migraine (Markowitz et al., 1987; Section 5.2.3), stroke (Fumagalli et al., 2011; McKittrick et al., 2015) and cancers (Grisold and Grisold, 2014). Although meningitides caused by viruses (Hill et al., 2015; Vergnano et al., 2015), bacteria (Djukic et al., 2006; van de Beek et al., 2012), protozoa (see below), nematodes (Dimitriadou et al., 1997), and fungi [notably Cryptococcus (Tseng et al., 2012; Antinori et al., 2014)] are widespread, study of the infected meninges in situ in animal models has been 
limited so far to a few diseases. Recruitment of immune cells to the meninges in the viral infection lymphocytic choriomeningitis (Kang and McGavern, 2009) has been imaged through the thinned skull by Kim et al. (2009). Meningeal lymphocyte activity induced by the protozoans Plasmodium berghei (malaria, Shaw et al., 2015), and Trypanosoma brucei (sleeping sickness, Mott, 1906 and Coles et al., 2015) has also been imaged in vivo. Other animal studies of infectious meningitis have mainly been based on histochemistry of the isolated dura [e.g., Nippostrongylus brasiliensis (Dimitriadou et al., 1997)], or analysis of CSF [e.g. Streptococcus pneumoniae (Polfliet et al., 2001)]. The meninges can harbor a major reservoir of the human immunodeficiency virus (Lamers et al., 2011). In uninfected animals, comparison of different studies suggests that laboratory rats (typically Lewis rats) may have more resident immune cells in the meninges than do laboratory mice, which are typically inbred C57BL/6 (see, e.g., Fig.9A-D).

Characterization of immune cells, particularly in the mouse, by use of a large array of transcriptomic markers (Cardona et al., 2006a; Becher et al., 2014; Hume and Freeman, 2014) has enormously increased the number of sub-types and put the nomenclature in flux (Hume et al., 2013; Randolph and Merad, 2013; Guilliams et al., 2014; Perie and Naik, 2015; Rua and McGavern, 2015). To be useful, analysis of the inflammatory transcriptomes of sorted cells must be correlated with the locations of the cells. Aided by use of reporter mice, this is beginning to be done for populations of intracranial immune cells (Engstrom et al., 2012; Vasilache et al., 2015) and is complemented by in vivo or ex vivo imaging of immune cell movements. But detailed analysis of meningeal immune cells is in its infancy, and here we mainly focus on the presence and behavior of cells classified with a conservative nomenclature.

For the few different meningitides in which immune cells have been studied, they have been found predominately either in the dura or in the leptomeninges, so it is important to distinguish these layers. Whether immune cells can transmigrate between these layers across the arachnoid membrane is an open question.

8.1. Mast cells. Neumann (1890) attributes the name 'mast cell' to Ehrlich, who labelled cells in various connective tissues, including the rat dura, that he considered a type of plasma cell and which became known as mast cells. Mast cells are widespread in connective tissue, particularly at host-environment interfaces, such as skin, but are rare in the cortical parenchyma (Dropp, 1972; Goldschmidt et al., 1984; Johnson and Krenger, 1992). They can be readily stained, classically and ubiquitously, with toluidine blue (Dropp, 1972). They contain, or can generate, at least 20 mediators, chemokines and cytokines (Marom and Casale, 1983; Ribatti, 2015) and are very heterogeneous. They can be partly classified by fluorescence histochemistry of their contents, such as heparin, histamine and 5-HT (Dimlich et al., 1991), and further by immunocytochemistry of proteins (Dimitriadou et al., 1997).

Mast cells are numerous in the cortical meninges. In rat, they are mainly found in the layer dissected from the top of the meninges after removal of the calvaria and are distinctively arranged along the middle meningeal artery and its branches, and along dural nerve bundles (Dimlich et al., 1991) and are therefore clearly in the dura. In mouse, mast cells are present in the dural layer that remains attached to the calvaria, with a smaller population in the pia (Sayed et al., 2010). Other authors have emphasized a mast cell population in the leptomeninges (Dropp, 1972; Silver and Curley, 2013) but without directly comparing the leptomeninges with the dura.

In rat, subtypes of dural mast cells can be identified by their protease content: rat mast cell protease I (RMCPI), RMCPII, or both proteases (RMCPI/II). In the cortical dura of uninfected rats, the commonest mast cells are RMCPI immunoreactive cells, called 'connective tissue' mast cells, which outnumber RMCPII (mucosal mast cells) cells eleven to one, (RMCPI/II) cells being even rarer (Dimitriadou et al., 1997; Fig. 9A). Infection by the nematode Nippostrongylus braziliensis, which is frequently used to induce a mast cell hyperplastic response, expands the population of the minority RMCPII by some fifteen fold (Dimitriadou et al., 1997; Fig.9B).

Most dural mast cells are closely apposed to nerve fibers containing CGRP or SP (Dimitriadou et al., 1997) and by using capsaicin to cause local denervation, or antagonists to $\mathrm{H}_{3}$ 
receptors, Dimitriadou et al. (1997) showed that the relationship is functional. Mast cell mediators are released by stimulation of the trigeminal nerve and, conversely, degranulation can cause prolonged $(>4 \mathrm{~h})$ activation of trigeminal nociceptor fibers, suggesting a role of mast cells in the pathogenesis of migraine (Levy et al., 2007; Pedersen et al., 2015). In mouse EAE (Christy et al., 2013) and a model of stroke (McKittrick et al., 2015), mast cells promote infiltration by neutrophils. In other tissues, mast cells have been shown to mediate T-cell recruitment (Mekori and Metcalfe, 1999).

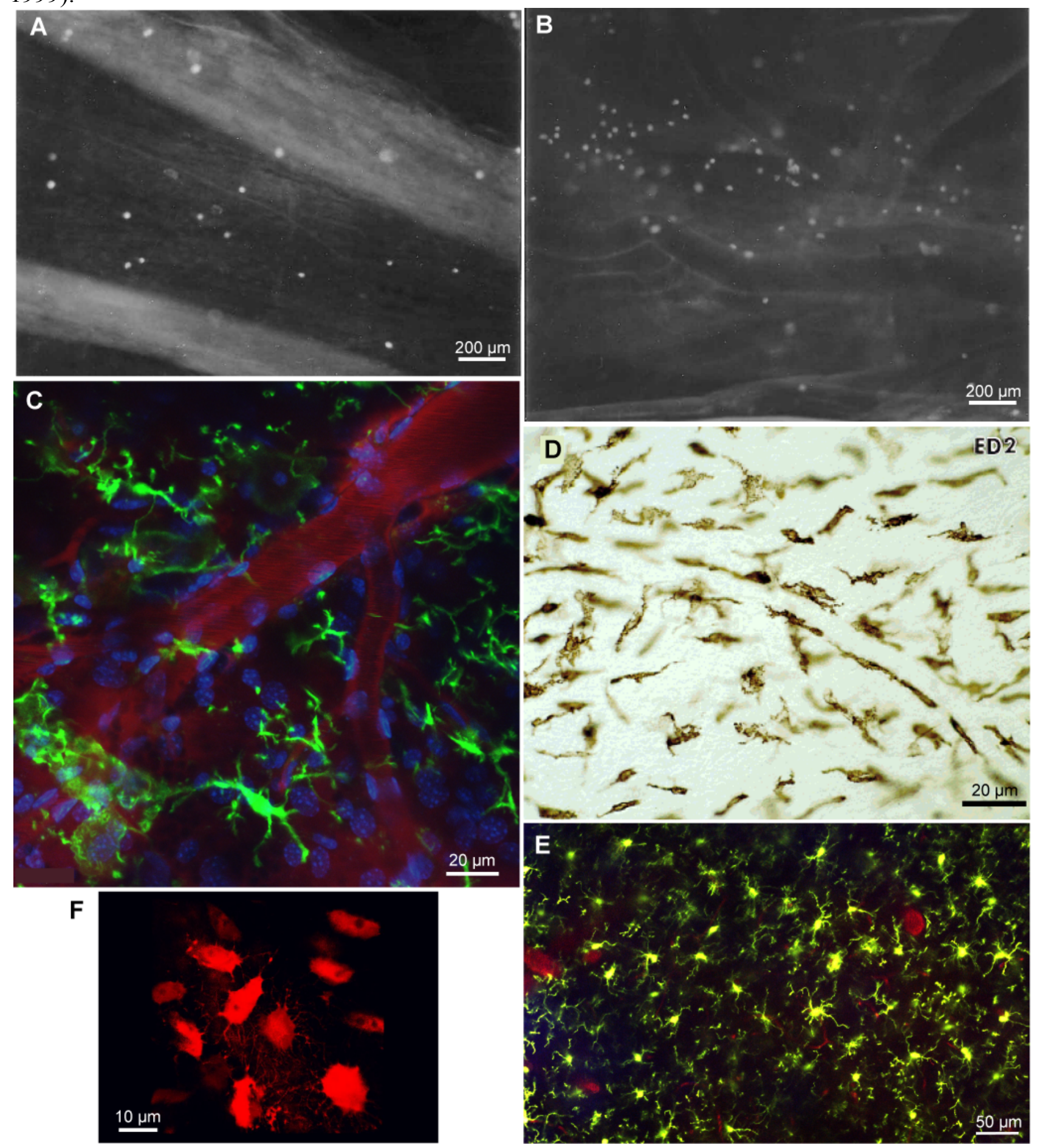

Figure 9. Mast cells, and cells of the macrophage family in the cortical meninges of Murinae.

A, B. Rat mast cells with immunoreactivity to rat mast cell protease II (RMCPII) in dural wholemounts. A. Uninfected. B. Six days after infection by the nematode Nippostrongylus braziliensis Reprinted from Dimitriadou et al., 1997, with permission from IBRO. C. Intravital transcranial two-photon microscopy of a C57BL/6 mouse expressing GFP under control of the $\mathrm{CX} 3 \mathrm{CR} 1$ promoter. $\mathrm{CX}_{3} \mathrm{CR} 1+$ cells (green) at the level of pial vessels. Vascular endothelial cells (blue) were labeled by i.v. injection of furamidine. D. CD163+ macrophages in a dural wholemount (Lewis rat). From 
McMenamin, 1999, with permission from Wiley-Liss. E. CX ${ }_{3}$ CR1+ microglia (yellow) in the superficial parenchyma. Penetrating blood vessels are labeled with rhodamine-dextran. F. Putative mouse osteoclasts imaged transcranially and labelled by superfusing the thinned skull with sulforhodamine B.

\subsection{Neutrophils}

Neutrophils, defined as CD11b+ CD11c- Ly6G+ (Daley et al., 2008), are the most common type of polymorphonuclear leukocytes (McColl et al., 1998) or polymorphonuclear granulocytes (Enzmann et al., 2013). They rapidly infiltrate skin challenged by irritants (Page and Good, 1958), and in EAE are recruited to the dura within a day (Christy et al., 2013), the recruitment depending on TNF produced by mast cells (Sayed et al., 2010). This is followed by recruitment to brain tissues (Walker-Caulfield et al., 2015). After infection with lymphocytic choriomeningitis (LCM) virus, in vivo imaging shows neutrophils infiltrating a layer extending a short distance from the skull downwards and, at later times, in the PVS of penetrating vessels (Kim et al., 2009). After transient middle cerebral artery occlusion (MCAO), the pattern is different. Neutrophils are recruited first to the leptomeninges, both inside vessels and closely associated with the abluminal surface, then appear mainly arrested within penetrating vessels, (Enzmann et al., 2013). They do not enter the parenchyma. However, after permanent, instead of transient, MCAO, Perez-de-Puig et al. (2015) found that after leptomeningeal invasion, neutrophils did enter the parenchyma. Injury by compression also causes neutrophil recruitment to the meninges (Roth et al., 2014).

\subsection{Microglia.}

The only numerous and well-documented immune cells within the healthy neural parenchyma are microglia (Ransohoff and Engelhardt, 2012; Brendecke and Prinz, 2015). In $\mathrm{CX}_{3} \mathrm{CR} 1-\mathrm{GFP}+$ reporter mice (Jung et al., 2000 and Cardona et al., 2006b), GFP+ microglia with fine ramifications and limited displacements are seen to be widespread, but densest just under the pia (Fig. 9E). In response to brain inflammation or trauma, microglia retract their ramifications to acquire a macrophage-like morphology, travel rapidly towards damage, and may become phagocytic (RioHortega, 1919; Kreutzberg, 1996; Kettenmann et al., 2011). Adult microglia are clearly distinct from (other) macrophages: they derive from primitive myeloid precursors that arise in the yolk sac early during embryonic development, express genes not expressed by other macrophages (Greter et al., 2015) and they self-maintain within the parenchyma with very little recruitment of blood-borne myeloid precursors (Theele and Streit, 1993; Djukic et al., 2006; Ajami et al., 2007; Ginhoux et al., 2010; Bruttger et al., 2015; Greter et al., 2015). However, in disease, circulating monocytes infiltrate the parenchyma and acquire phenotypes similar that of resident microglia (Flugel et al., 2001; Djukic et al., 2006; Gomez-Nicola et al., 2014; Greter et al., 2015). Depending on the conditions, circulating monocytes may (Varvel et al., 2012), or may not (Bruttger et al., 2015), contribute to restoring a microglia population that has been artificially depleted.

\subsection{Perivascular cells}

Pericytes and perivascular macrophages are located between the endothelium of penetrating vessels and the vascular basal lamina facing the perivascular space. Pericytes are contractile (FernandezKlett et al., 2010) and perivascular macrophages are phagocytic (Kida et al., 1993b; Mato et al., 1996). Transcriptome analysis suggests that brain perivascular macrophages participate in the response to intraperitoneal injection of bacterial wall lipopolysaccharide (LPS) at 3h by modifying prostaglandin metabolism and producing interleukin-6 (Vasilache et al., 2015) but there seems to be no good evidence that they translocate into the parenchyma: Kida et al. (1993b) labeled perivascular macrophages with phagocytosed carbon particles and found some, at least, of these cells still in place two years later. In contrast, after occlusion of the middle cerebral artery, pericytes within the infarct core proliferate, invade the parenchyma, and express microglial markers (Ozen et al., 2014); this observation was greatly helped by use of reporter mice expressing GFP under control of the pericyte-specific RSG5 promoter.

\subsection{Meningeal macrophages}


Immunocytochemistry on dural wholemounts of mice and rats has shown macrophages heterogeneous in their expression of antigens. The antigens CD163 ${ }^{+}$(group B scavenger receptor, ED2 clone; Fig. 9D), $\mathrm{CD}^{+} 8^{+}$(ED1, lysosomal marker) and CD169 ${ }^{\text {low }}$ (Siglec 1, sialoadhesin, ED3 clone) are widespread and some cells express two or more of these. A few express MHC class II (McMenamin, 1999; Zhang et al., 2009; Chinnery et al., 2010). In CX3CR1-GFP+ myeloid-specific reporter knock-in mice (Jung et al., 2000), both subpial microglia (Fig. 9E) and a population of meningeal macrophages are fluorescent (Chinnery et al., 2010). From their shapes and positions within the meninges, $\mathrm{CX}_{3} \mathrm{CR} 1+$ cells may include a sub-population of CD163+ cells (Fig.9C). Large CD68+ CD163- cells identified as osteoclasts (Sminia and Dijkstra, 1986) are visible in dural wholemounts, distributed along the length of meningeal vessels (McMenamin, 1999; Chinnery et al., 2010). Osteoclast-like cells can also be visualized in vivo by their ability to take up sulforhodamine superfused over the thinned skull (Fig. 9F).

In rats, lipopolysaccharide (LPS) increases the number of meningeal CD68+ cells (Yamate et al., 2009). In mice infected by lymphocytic choriomeningitis virus, 'pathogenic monocytes' are recruited to a meningeal space including the dura (Kim et al., 2009) and macrophages isolated from the dura are more active in those mice destined to survive (Woods et al., 1987). Shaw et al. (2015) show that $\mathrm{CX}_{3} \mathrm{CR} 1^{+/ \mathrm{GFP}}$ cells, which include populations of inflammatory monocytes, macrophages and dendritic cells, interact with $\mathrm{T}$ cells adjacent to pial vessels in mice infected with the malaria parasite Plasmodium berghei. In mice infected with the protozoan Trypanosoma brucei large numbers of cells expressing CD11c appear in the dura (Fig.10E). At first identifed as dendritic cells (Coles et al., 2015), these may in fact be macrophages.

\subsection{Dendritic cells.}

Classical dendritic cells (cDCs) are derived from bone marrow myeloid progenitors that undergo progressive lineage restriction but include many subtypes. Their defining functional attributes, such as possession of a "superior antigen processing and presentation machinery" and a "superior ability to migrate loaded with tissue antigens to the T cell zone of lymph nodes"(Merad et al., 2013) are not easily demonstrated on resident intracranial cells and the identification of intracranial DCs depends heavily on detection of molecular markers. Of these, the major histocompatibility complex (MHC) Class II and the integrin CD11c, have been used, but these markers are also expressed by other cell types, notably macrophages (Lindquist et al., 2004; Immig et al., 2015). A marker that does appear to be specific to cDCs is the zinc finger Zbtb46 (BTBD4; Meredith et al., 2012; Satpathy et al., 2012). Zbtb46 ${ }^{\mathrm{GFP} /+}$ mice have been constructed, but we find no results on brain published to date. Progenitor DCs also appear to be unique in expanding in response to FMS-like tyrosine kinase 3 ligand (flt3L; Anandasabapathy et al., 2011).

Despite the ambiguity of the markers used, there is general agreement that there are almost no dendritic cells in the cortical parenchyma of mice. In CD11c-eYFP mice, in which myeloid dendritic cells and a subpopulation of macrophages are fluorescent (Lindquist et al., 2004), the few fluorescent cells in the brain parenchyma are at the glia limitans (Prodinger et al., 2011), display the microglial markers IBA-1 and CD11b (Immig et al., 2015), and are not expanded by treatment of the mouse with flt3L, a defining characteristic of dendritic cells (Anandasabapathy et al., 2011). Using in vivo two-photon microscopy through the skull, no CD11c -eYFP cells were observed in the superficial cortex (Fig. 10F). Elsewhere in the brain, Steinmann and colleagues (Bulloch et al., 2008) report the presence of CD11c-eYFP cells in regions that lack a BBB or are sites of neurogenesis, and call these "brain dendritic cells".

In dural wholemounts of Lewis rats, numerous MHC Class II cells (revealed with the monoclonal antibody OX6) are found in the inner layer and connective tissue of the dura, and, in at least equal numbers, in the arachnoid membrane (Fig.10A-C; McMenamin, 1999; McMenamin et al., 2003). The dendriform and pleomorphic morphology of these cells and their regular, nonperivascular, distribution suggest these cells are of the DC lineage. Cells with similar characteristics are found in a dense regular network in the pia (McMenamin, 1999; McMenamin et al., 2003). In dural wholemounts from C57BL/6 CD11c-eYFP reporter mice there is a sparse 
population of $\mathrm{CD} 11 \mathrm{c}+$ cells, representing $23 \%$ of the $\mathrm{MHCII}+$ population, and a denser one in the pia mater (62\% of the MHCII+ population) (Chinnery et al., 2010). In in vivo two-photon imaging, the very rare pial CD11c-YFP+ cells are round and juxtavascular (Fig. 10F), suggesting they are macrophages. However, in the dura, there are occasional CD11c-YFP+ cells with the morphology and movement of dendritic cells (Fig.10D; Coles et al., 2015).

The number of CD11c-YFP+ cells in the dura increases manyfold in mice infected with the protozoan Trypanosoma brucei. These cells have an amoeba-like morphology and interact with $\mathrm{T}$ cells in situ and may be described as macrophages.

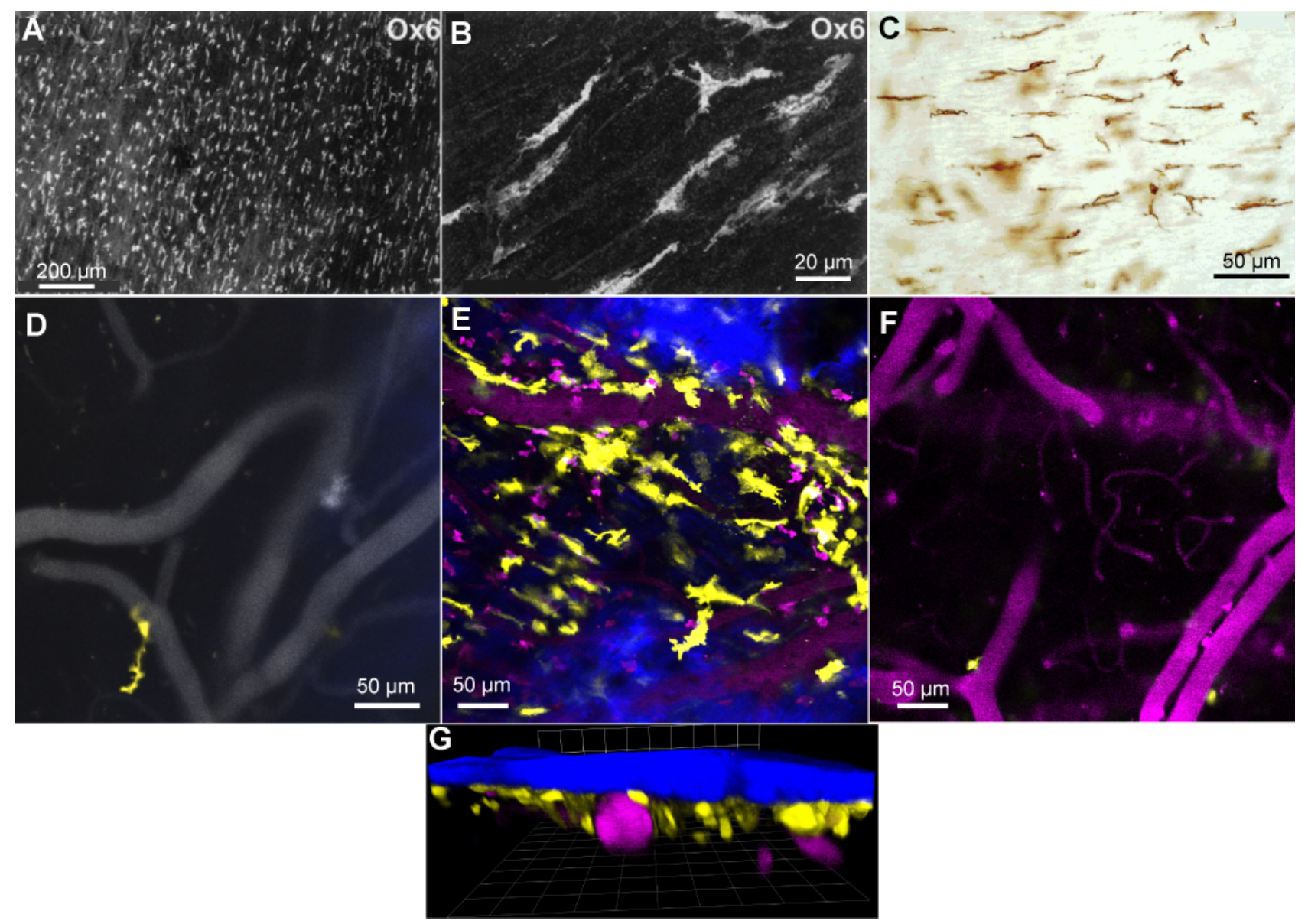

Figure 10. Dendritic cells in the cortical meninges. $(\mathbf{A}, \mathbf{B}, \mathbf{C})$ Dendritic cells in Lewis rats. $(\mathbf{A}, \mathbf{B}) \mathrm{Ox6}+(\mathrm{MHCII}+)$ cells seen from the inner aspect of a dural wholemount including outer layers of the arachnoid, at two magnifications. Environmental scanning electron micrographs modifed from McMenamin et al. 2003. (C) On the endosteal aspect of a dural wholemount, Ox6+ cells are among collagen fibers. Modifed from McMenamin, 1999. (D-G) Transcranial intravital two-photon microscopy of C57BL/6 mice expressing YFP under control of the CD11c promoter. (D) A dendritic cell on a pial vessel of an uninfected mouse. The video of this cell shows extension and retraction of processes (S1 Video in Coles et al., 2015). (E) Numerous CD11c+ cells close to the skull (blue) 12 days after infection with Trypanosoma brucei brucei. Pink objects may be macrophages that have ingested quantum dots used as the blood marker. (F) A deeper level of the same XY field as (E) showing horizontal vessels on the pia and vertical vessels penetrating the parenchyma. Two CD11c+ cells are visible. (G) A 3D reconstruction of a Z-stack $139 \mu \mathrm{m}$ deep 27 days after infection with T.b.brucei. The grid spacing is $42.4 \mu \mathrm{m}$. (D-G) from Coles et al., 2015.

8.8. $T$ cells. $T$ cells are sparse in the uninflamed leptomeninges (Kivisakk et al., 2009; Fumagalli et al., 2011; Coles et al., 2015; Shaw et al., 2015), rarer in the dura, and not seen in the superficial parenchyma (Fig. 11ABC; Coles et al., 2015). Small numbers of central memory CD4 ${ }^{+} \mathrm{T}$ cells enter CSF from the choroid plexus and pial venules (Kivisakk et al., 2003; Reboldi et al., 2009) and are carried by CSF flow through the subarachnoid space as a normal part of immunosurveillance against CNS infections (Ransohoff et al., 2003; Ransohoff and Engelhardt, 2012); naive or resting T cells do not reach the CSF (Kivisakk et al., 2003). Recruitment of activated T cells to brains of mice can differ by a factor of four between standard laboratory strains (Carrithers et al., 2002). In 
three pathologies for which data are available, an increase in the number of $T$ cells occurs in the leptomeninx. The MCAO stroke model significantly increases the number of $\mathrm{T}$ cells in the mouse cortical leptomeninx (Fig. 11D;Fumagalli et al., 2011), and infection by the malaria parasite, Plasmodium berghei, causes a much greater increase (Fig. 11E; Shaw et al., 2015). EAE also causes proliferation of $\mathrm{CD}^{+}{ }^{+} \mathrm{T}$ cells in the mouse leptomeninx (Kivisakk et al., 2009), and, in rat spinal cord, cytotoxic T cells, after 'licensing' in the lung (Odoardi et al., 2012) crawl within pial vessels and have been observed leaving them by diapedesis (Bartolomäus et al., 2009). Having left the pial vessels, these T cells show calcium-dependent activation (Pesic et al., 2013), then disperse through the leptomeningeal space (Bartolomäus et al., 2009; Odoardi et al., 2012), before moving down the perivascular spaces of penetrating vessels (Bartolomäus et al., 2009). The behavior of extravascular $\mathrm{CD}^{+}{ }^{\mathrm{T}}$ cells in mouse cranial leptomeninx (after MCAO or Plasmodium berghei infection) is different from that in spinal cord (in EAE) in that extravascular T cells patrol close to the vessels (Fumagalli et al., 2011; Shaw et al., 2015). A factor that might contribute to the tendency to perivascular localization in the leptomeninx of mouse brain, compared to rat spinal cord, is that the extravascular space appears to be limited to channels alongside the vessels (Fig.6A-F). The usefulness of in vivo imaging is illustrated by the results of Shaw et al. (2015) who found that although two strains of $P$. berghei caused equal meningeal recruitment of T cells whose populations were indistinguishable by traditional flow cytometry, the movements of the extravascular $\mathrm{T}$ cells were different: after infection by one strain (P.berghei ANKA), T cells were arrested more often than with the other (P.berghei NK65).

In the three pathologies just described (ischemia, EAE and malaria), the increase in $\mathrm{T}$ cells occurs mainly in the leptomeninx (although in rat spinal cord a few T cells may be in the dura (Fig. 10F; Bartolomäus et al., 2009). In contrast, in infections by Trypanosoma brucei, $\mathrm{T}$ cell infiltration appears to be localized to the dura: the T cells are close to the skull, at a level where capillaries and collagen are present (Fig. 12A-D; Coles et al., 2015). Unlike the T cells observed in the mouse cortical leptomeninx, the movements of $\mathrm{T}$ cells in the dura appear to be unrelated to blood vessels (Fig. 12E), in keeping with the absence of paravascular channels like those in the leptomeninx. Kim et al. (2009) have also reported that in mice infected with lymphocytic choriomeningitis virus cytotoxic $\mathrm{T}$ lymphocytes are recruited to a meningeal space close under the skull where they moved independently of the blood vessels. We suggest that they were in the dura. However, in both pathologies, $T$ cells appear to reach the perivascular spaces of penetrating vessels, which implies crossing the arachnoid barrier layer. 

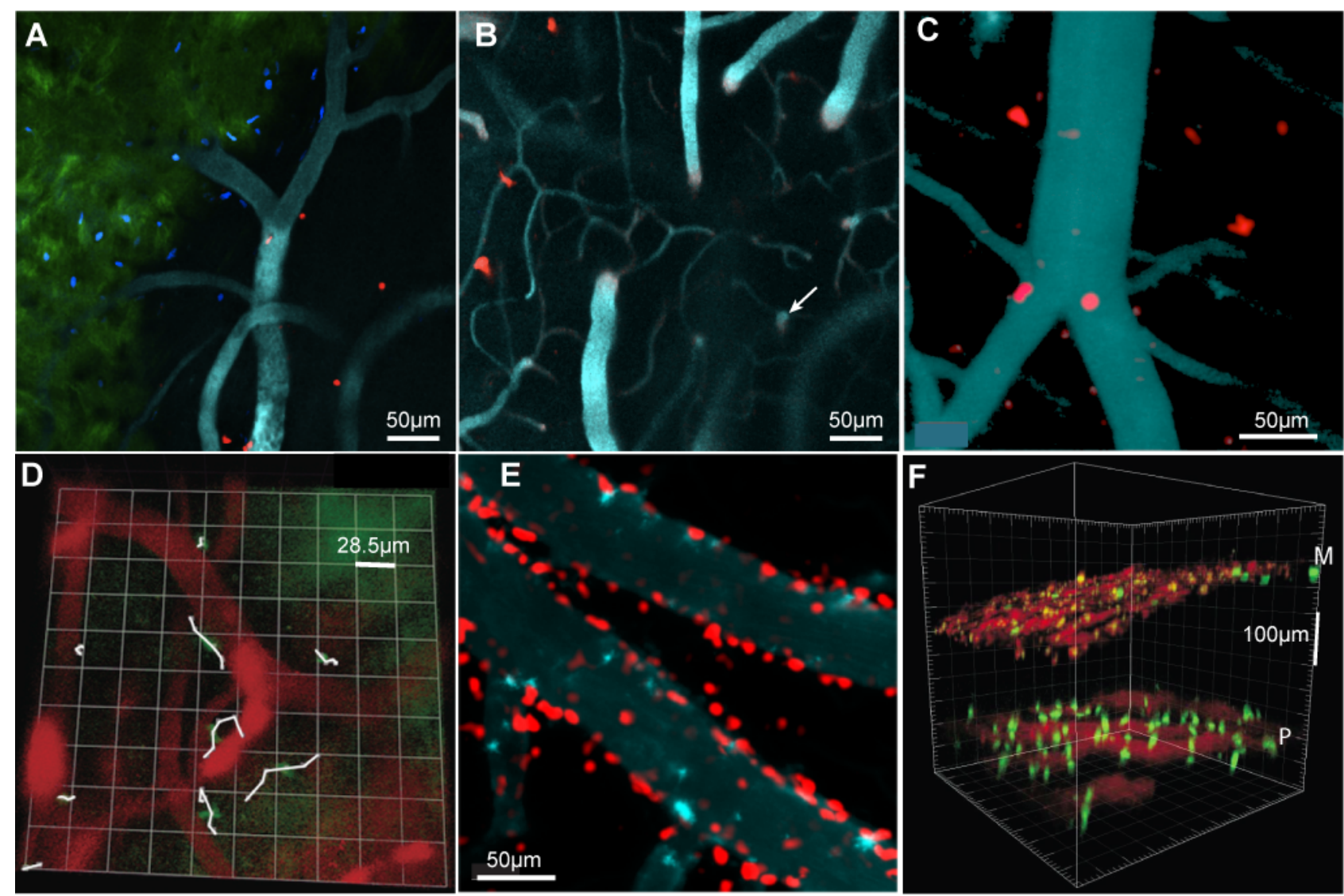

Figure 11. $\mathbf{T}$ cells in the leptomeninx. Transcranial intravital two-photon images of C57BL/6 mice expressing DsRed (or GFP) under control of the CD2+ promoter. A,B,C. T cells are rare in the meninges of uninfected mice. A. A slightly oblique $20 \mu \mathrm{m}$ Z-projection, showing, upper left, skull bone (green) and nuclei of dural cells (blue) and T cells (red) slightly deeper. B. Another $20 \mu \mathrm{m}$ Z-projection from the same Z-stack as (A). Pial vessels are seen abruptly plunging into the cortex, where vertical vessels (arrow) are linked by sinuous capillaries. C. In another mouse, a few $\mathrm{T}$ cells are present close to leptomeningeal vessels. From Shaw et al., 2015. D. Tracks of CD2+ GFP T cells over 15 min in a C57BL/6 mouse 3 days after permanent middle cerebral artery occlusion. From Fumagalli et al., 2011. E. Much increased presence of $\mathrm{T}$ cells outside pial vessels in a model of encephalytic cerebral malaria ( 7 days after infection with Plasmodium berghei ANKA). From Shaw et al., 2015. F. EAE in rat spinal cord imaged after removal of the bone. $\mathrm{CD} 2+$ GFP T cells in green. (P) pial vessels with many T cells. (M) dural vessels with a few T cells. Modified from Bartholomäus et al., 2009, Fig S1b. 

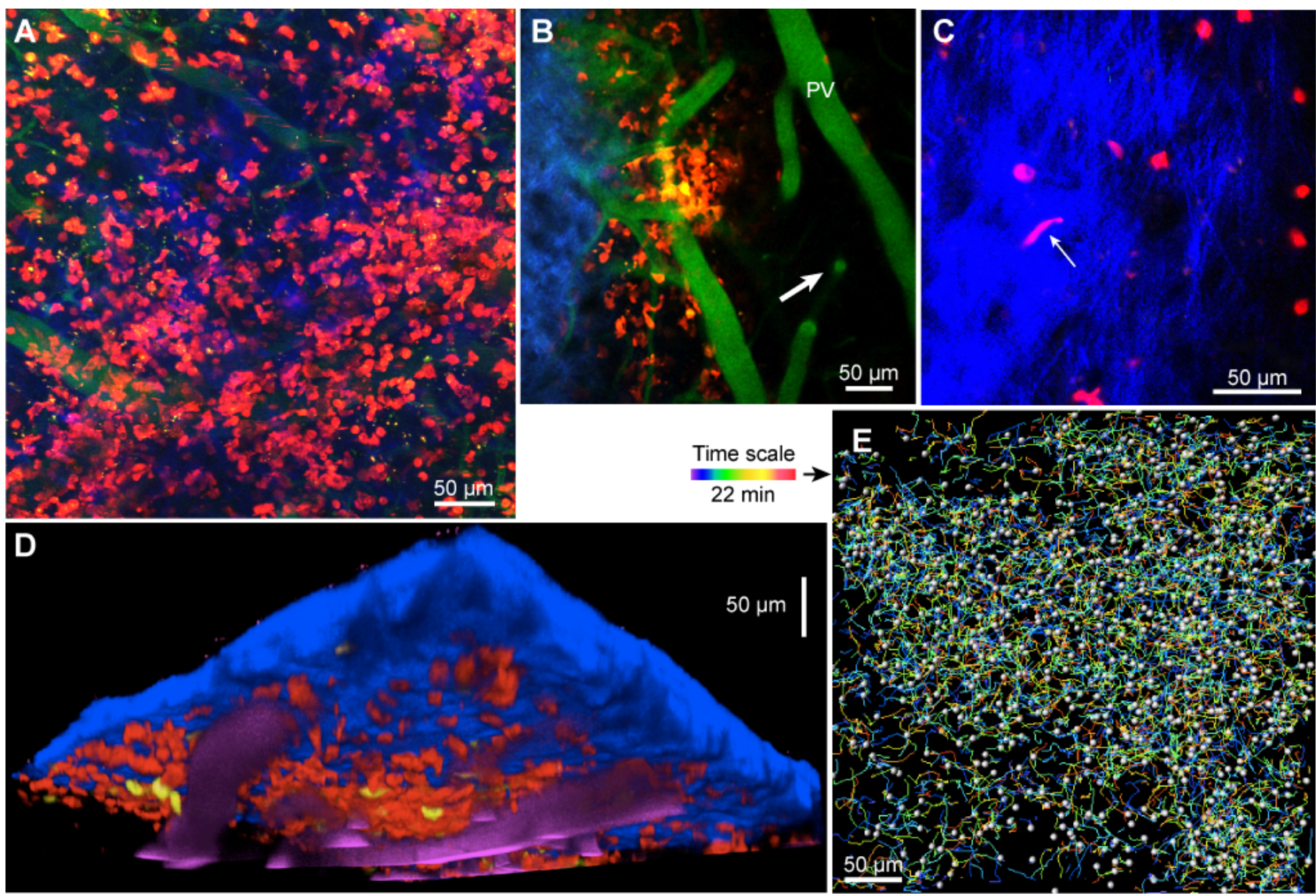

Figure 12. $T$ cells in the dura after infection by Trypanosoma brucei brucei. A. T cells (CD2+ DsRed) in the meninges of a mouse 39 days post infection (dpi). Extended focus view of a Z-stack $188 \mu \mathrm{m}$ deep. Faint green is blood plasma, faint blue is collagen and furamidine labeled nuclei. B. A single, slightly oblique, plane, showing numerous $\mathrm{T}$ cells close to skull bone (blue, left) and small blood vessels, but few on large pial vessels ('PV') and none on vertically penetrating vessels (e.g., arrow). (12 dpi). C. Frame from a video in which a $\mathrm{T}$ cell (arrow) can be seen moving among collagen fibers (blue). (11 dpi). D. A 3D reconstruction from a Z-stack showing $\mathrm{T}$ cells mainly above the large pial vessels (magenta). Yellow cells are CD11c+ YFP. (12 dpi) E. Tracks of T cells in the dura. Z-stacks, $75 \mu \mathrm{m}$ thick, were obtained repeatedly over $22 \mathrm{~min}$. Time along each track is color-coded from violet to red (timescale bar). The grey balls show the positions of the cells at 9 min. (40 dpi) Figures from Coles et al. 2015.

\subsection{Antigen presentation in the meninges.}

In the uninflamed brain, most of the small number of $T$ cells that enter CSF appear to have been activated elsewhere (Kivisakk et al., 2003) but, in disease, antigens are presented to $\mathrm{T}$ cells in the meninges. During the initiation of EAE, long-lasting interactions between MHCII+ antigenpresenting cells and T cells are observed in the leptomeninx, and T cells proliferate (Bartolomäus et al., 2009 and Kivisakk et al., 2009).

In mice infected with lymphocytic choriomeningitis virus, antigen presentation from MHCII+ cells to T cells is observed in the meninges (Lauterbach et al., 2006; Kim et al., 2009). And in mice infected with Trypanosoma brucei, long-lasting interactions between CD11c + cells (probably macrophages) and T cells have been observed in the dura (Coles et al., 2015). In contrast, Shaw et al. (2015) found no evidence of antigen presentation in the meninges of mice with experimental cerebral malaria.

\subsection{Communication between the dura and CSF.}

The cells of the arachnoid membrane are connected by tight junctions (Nabeshima et al., 1975) and, at least in healthy animals, dye does not pass from dura to subarachnoid space (Rodriguez-Peralta, 1957; Balin et al., 1986) or from SAS to dura (Figs 6A-G) and the extracellular fluid in the dura and the CSF in the SAS are different (Cserr and Bundgaard, 1984). Despite this, experimental results 
indicate some communication between the dura and CSF, and raise questions about how such communication might occur.

Signaling from brain cortex to dura is observed during spreading depression (Section 5.2.3) when neuronal malfunction in the gray matter leads to release of inflammatory mediators into the leptomeninx, and also causes plasma protein extravasation in the dura (Bolay et al., 2002; Pietrobon and Moskowitz, 2014). It has been shown that infusion of inflammatory mediators into the leptomeninx can excite trigeminal neurons (Ebersberger et al., 1997,1999b), so one possibility is that there is a centripetal-centrifugal neuronal pathway, from leptomeninx to trigeminal ganglion to dura (Bolay et al., 2002 and Messlinger, 2009) or an axon reflex (Pietrobon and Moskowitz, 2014) in fibers with collateral branches in the dura and leptomeninx. Signaling (in the reverse direction, from dura to leptomeninx) appears to occur when activation of mast cells in the dura facilitates early T cell infiltration of the brain parenchyma in EAE (Sayed et al., 2010), and when parenchymal sequelae of ischemia are moderated in mice deficient in dural mast cells (Arac et al., 2014; McKittrick et al., 2015). Again, this signaling might be subserved by branches of the trigeminal nerve.

However, a circuitous neuronal pathway between dura and leptomeninx will not explain recent observations on intracranial lymph vessels. Aspelund et al. (2015) report that although the lymph vessels are in the dura (Andres et al., 1987) they "absorb CSF from the adjacent subarachnoid space", in agreement with Louveau et al. (2015), who state that the dural lymph vessels are "able to carry both fluid and immune cells from the cerebrospinal fluid". In the reverse direction, it appears possible that $\mathrm{T}$ cells can migrate from the dura into the perivascular space of penetrating arterioles (Kim et al., 2009 and Coles et al., 2015). The mechanisms of this exchange are unclear. The arachnoid barrier layer shares features with the blood-brain barrier at the vascular endothelium of intracerebral vessels: it is at the interface of two fluid compartments, it has tight intercellular junctions, its cells are rich in mitochondria (Fig. 4A; Pease and Schultz, 1958; Oda and Nakanishi, 1984), and they express drug transporting molecules (Yasuda et al., 2013). Like the capillary blood-brain barrier, the arachnoid barrier layer may well be the site of intense transcellular exchange. (Mercier and Hatton, 2000) and (Kosaras et al., 2009) have reported other anatomical features that, they speculate, might subserve communication across the arachnoid membrane. Mercier and Hatton conclude from the pattern of immunolabeling of the calcium-binding protein, S100 $\beta$, and the filament vimentin, that a network of cell processes penetrate the arachnoid membrane. Kosaras et al reported nerve fibers containing CGRP and stretching directly between the dura and pia.

\section{Conclusion.}

Immune dynamics in the dura, in the leptomeninx, and in the parenchyma can be very different, and it is now known that, in different diseases, the site of greatest immune activity within the meninges can be either the dura or the leptomeninx (Table 3). Transcranial intravital imaging allows analysis of the numbers and movements of cells, while careful tissue separation potentially allows detailed analysis of cell types in each compartment. Despite much new information, big questions remain concerning the functional anatomy of the meninges and the perivascular sheaths, and the movement of fluids and cells within and between the various extracellular compartments, particularly across the arachnoid 'barrier' membrane. 
Table 3. Changes in the Immune Cell Population of the Cortical Meninges in various Pathologies

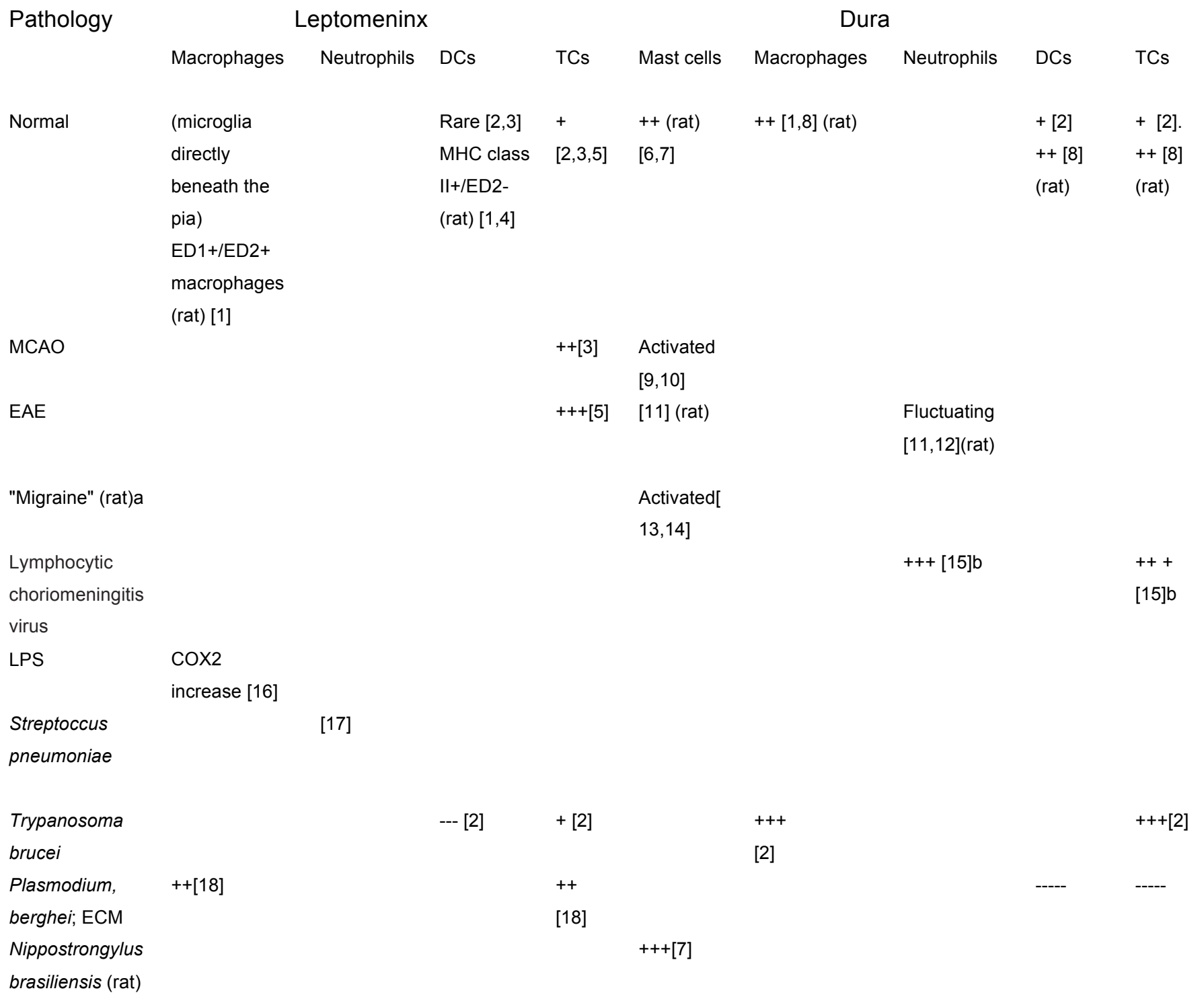

Data from mouse except where stated. MCAO: Middle cerebral artery occlusion. ECM: Experimental cerebral malaria models.

(a) "Migraine": stimulation of the trigeminal nerve or application of CGRP. (b) The dural location is our interpretation of the published images.

1. McMenamin (1999). 2. Coles, et al. (2015). 3. Fumagalli et al. (2011). 4. Quintana et al. (2015). 5. Kivisakk, et al. (2009). 6. Motavkin, et al. (1979). 7. Dimitriadou, et al. (1997). 8. McMenamin, et al. (2003). 9. Arac, et al. (2014). 10. McKittrick, et al. (2015). 11. Christy, et al. (2013). 12. Walker-Caulfield, et al. (2015). 13. Dimitriadou, et al. (1991).

14. Schwenger, et al. (2007). 15. Kim, et al. (2009).16. Elmquist, et al. (1997). 17. Gerber, et al. (2001). 18. Shaw, et al. (2015).

\section{References}

Abbott N.J., Patabendige, A.A., Dolman, D.E., Yusof, S.R., Begley, D.J., 2010. Structure and function of the blood-brain barrier. Neurobiol. Dis. 37, 13-25.

Adam, J.F., Nemoz, C., Bravin, A., Fiedler, S., Bayat, S., Monfraix, S., Berruyer, G., Charvet, A.M., Le Bas, J.F., Elleaume, H., Esteve, F., 2005. High-resolution blood-brain barrier permeability and blood volume imaging using quantitative synchrotron radiation computed tomography: study on an F98 rat brain glioma. J. Cereb. Blood Flow Metab. 25, 145-153. 
Ajami, B., Bennett, J.L., Krieger, C., Tetzlaff, W., Rossi, F.M., 2007. Local self-renewal can sustain CNS microglia maintenance and function throughout adult life. Nat. Neurosci. 10, 1538-1543.

Akerman, S., Goadsby, P.J. 2015. Neuronal PAC1 receptors mediate delayed activation and sensitization of trigeminocervical neurons: Relevance to migraine. Science Trans. Med. 7, Article Number: 308 ra157.

Akerman, S., Williamson, D.J., Kaube, H., Goadsby, P.J., 2002a. The effect of anti-migraine compounds on nitric oxide-induced dilation of dural meningeal vessels. Eur. J. Pharmacol. 452, 223-228.

Akerman, S., Williamson, D.J., Kaube, H., Goadsby, P.J., 2002b. Nitric oxide synthase inhibitors can antagonize neurogenic and calcitonin gene-related peptide induced dilation of dural meningeal vessels. Br. J. Pharmacol. 137, 62-68.

Akerman, S., Williamson, D.J., Kaube, H., Goadsby, P.J., 2002c. The role of histamine in dural vessel dilation. Brain Res. 956, 96-102.

Alcolado, R., Weller, R.O., Parrish, E.P., Garrod, D., 1988. The cranial arachnoid and pia mater in man: anatomical and ultrastructural observations. Neuropathol. Appl. Neurobiol. 14, 1-17.

Alexander, W.T., 1875. Bemerkungen über die Nerven der Dura mater. Archiv für Mikroskopische Anatomie 11, 231 - 234.

Amenta, F., Sancesario, G., Ferrante, F., Cavallotti, C., 1980. Acetylcholinesterase-containing nerve fibers in the dura mater of guinea pig, mouse, and rat. J. Neural Transm. 47, 237-242.

Amin, F.M., Asghar, M.S., Hougaard, A., Hansen, A.E., Larsen, V.A., de Koning, P.J., Larsson H.B., Olesen J., Ashina M., 2013. Magnetic resonance angiography of intracranial and extracranial arteries in patients with spontaneous migraine without aura: a cross-sectional study. Lancet Neurol. 12, 454-461.

Anandasabapathy, N., Victora, G.D., Meredith, M., Feder, R., Dong, B., Kluger, C., Yao, K., Dustin, M.L., Nussenzweig, M.C., Steinman, R.M., Liu, K., 2011. Flt3L controls the development of radiosensitive dendritic cells in the meninges and choroid plexus of the steady-state mouse brain. J. Exp. Med. 208, 1695-1705.

Andres, K.H., 1967. [On the fine structure of the arachnoidea and dura mater of mammals]. Z. Zellforsch. Mikrosk. Anat. 79, 272-295.

Andres, K.H., von Duering, M., Muszynski, K., Schmidt, R.F., 1987. Nerve fibres and their terminals of the dura mater encephali of the rat. Anat. Embryol. (Berl.) 175, 289-301.

Angelov, D.N., Vasilev, V.A., 1989. Morphogenesis of rat cranial meninges. A light- and electronmicroscopic study. Cell Tissue Res. 257, 207-216.

Antinori, S., Peri, A.M., Milazzo, L., 2014. Fungal meningitis in England and Wales. Lancet Infect. Dis. 14, 921.

Appaix, F., Girod, S., Boisseau, S., Romer, J., Vial, J.C., Albrieux, M., Maurin, M., Depaulis, A., Guillemain, I., van der Sanden, B., 2012. Specific in vivo staining of astrocytes in the whole brain after intravenous injection of sulforhodamine dyes. PLoS One 7, e35169.

Arac, A., Grimbaldeston, M.A., Nepomuceno, A.R., Olayiwola, O., Pereira, M.P., Nishiyama, Y., Tsykin, A., Goodall, G.J., Schlecht, U., Vogel, H., Tsai, M., Galli, S.J., Bliss, T.M., Steinberg, G.K., 2014. Evidence that meningeal mast cells can worsen stroke pathology in mice. Am. J. Pathol. 184, 2493-2504.

Arbel-Ornath, M., Hudry, E., Eikermann-Haerter, K., Hou, S., Gregory, J.L., Zhao, L., Betensky, R.A., Frosch, M.P., Greenberg, S.M., Bacskai, B.J., 2013. Interstitial fluid drainage is impaired in ischemic stroke and Alzheimer's disease mouse models. Acta Neuropathol. 126, 353-364.

Arnold, F. (1831) Der Kopfteil des Vegetativen Nervensystems beim Menschen. Heidelberg: K. Groos.

Asghar, M.S., Hansen, A.E., Amin, F.M., van der Geest, R.J., Koning, P., Larsson, H.B., Olesen, J., Ashina, M., 2011. Evidence for a vascular factor in migraine. Ann. Neurol. 69, 635-645. 
Asgari, M., de Zelicourt, D., Kurtcuoglu, V., 2016. Glymphatic solute transport does not require bulk flow. Sci. Rep. 6, 38635.

Aspelund, A., Antila, S., Proulx, S.T., Karlsen, T.V., Karaman, S., Detmar, M., Wiig, H., Alitalo, K., 2015. A dural lymphatic vascular system that drains brain interstitial fluid and macromolecules. J. Exp. Med. 212, 991-999.

Attwell, D., Buchan, A.M., Charpak, S., Lauritzen, M., Macvicar, B.A., Newman, E.A., 2010. Glial and neuronal control of brain blood flow. Nature 468, 232-243.

Ayata, C., Dunn, A.K., Gursoy, O.Y., Huang, Z., Boas, D.A., Moskowitz, M.A., 2004. Laser speckle flowmetry for the study of cerebrovascular physiology in normal and ischemic mouse cortex. J. Cereb. Blood Flow Metab. 24, 744-755.

Baledent, O., Gondry-Jouet, C., Meyer, M.E., De Marco, G., Le Gars, D., Henry-Feugeas, M.C., Idy-Peretti, I., 2004. Relationship between cerebrospinal fluid and blood dynamics in healthy volunteers and patients with communicating hydrocephalus. Invest. Radiol. 39, 4555.

Balin, B.J., Broadwell, R.D., Salcman, M., el-Kalliny, M., 1986. Avenues for entry of peripherally administered protein to the central nervous system in mouse, rat, and squirrel monkey. $\mathrm{J}$. Comp. Neurol. 251, 260-280.

Barbier, E.L., Lamalle, L., Decorps, M., 2001. Methodology of brain perfusion imaging. J. Magn. Reson. Imaging 13, 496-520.

Barkhof, F., Kouwenhoven, M., Scheltens, P., Sprenger, M., Algra, P., Valk, J., 1994. Phasecontrast cine MR imaging of normal aqueductal CSF flow. Effect of aging and relation to CSF void on modulus MR. Acta Radiol. 35, 123-130.

Bartolomäus, I., Kawakami, N., Odoardi, F., Schlager, C., Miljkovic, D., Ellwart, J.W., Klinkert, W.E., Flugel-Koch, C., Issekutz, T.B., Wekerle, H., Flugel, A., 2009. Effector T cell interactions with meningeal vascular structures in nascent autoimmune CNS lesions. Nature 462, 94-98.

Baruch, K., Deczkowska, A., David, E., Castellano, J.M., Miller, O., Kertser, A., Berkutzki, T., Barnett-Itzhaki, Z., Bezalel, D., Wyss-Coray, T., Amit, I., Schwartz, M., 2014. Aginginduced type I interferon response at the choroid plexus negatively affects brain function. Science 346, 89-93.

Bateman, G.A., Brown, K.M., 2012. The measurement of CSF flow through the aqueduct in normal and hydrocephalic children: from where does it come, to where does it go? Childs Nerv. Syst. 28, 55-63.

Baun, M., Hay-Schmidt, A., Edvinsson, L., Olesen, J., Jansen-Olesen, I., 2011. Pharmacological characterization and expression of VIP and PACAP receptors in isolated cranial arteries of the rat. Eur. J. Pharmacol. 670, 186-194.

Bayliss, W.M., 1902. On the local reactions of the arterial wall to changes of internal pressure. J. Physiol. 28, 220-231

Bayliss, W.M., Hill, L., Gulland, C.L., 1895. On intra-cranial pressure and the cerebral circulation. Part I. Physiological. J. Physiol. 18, 344-362.

Becher, B., Schlitzer, A., Chen, J., Mair, F., Sumatoh, H.R., Teng, K.W., Low, D., Ruedl, C., Riccardi-Castagnoli, P., Poidinger, M., Greter, M., Ginhoux, F., Newell, E.W., 2014. Highdimensional analysis of the murine myeloid cell system. Nat. Immunol. 15, 1181-1189.

Bedussi, B., van Lier, M.G., Bartstra, J.W., de Vos, J., Siebes, M., VanBavel, E., Bakker, E.N., 2015. Clearance from the mouse brain by convection of interstitial fluid towards the ventricular system. Fluids Barriers CNS 12, 23.

Ben-Zvi, A., Lacoste, B., Kur E., Andreone, B.J., Mayshar, Y., Yan, H., Gu, C., 2014. Mfsd2a is critical for the formation and function of the blood-brain barrier. Nature 509, 507-511.

Bennett, M.V., 1969. Electrical impedance of brain surfaces. Brain Res. 15, 584-590.

Bergerot, A., Reynier-Rebuffel, A.M., Callebert, J., Aubineau, P., 2000. Long-term superior cervical sympathectomy induces mast cell hyperplasia and increases histamine and serotonin content in the rat dura mater. Neuroscience 96, 205-213. 
Bergstrand, G., Bergstrom, M., Nordell, B., Stahlberg, F., Ericsson, A., Hemmingsson, A., Sperber, G., Thuomas, K.A., Jung, B., 1985. Cardiac gated MR imaging of cerebrospinal fluid flow. J. Comput. Assist. Tomogr. 9, 1003-1006.

Bering, E.A., Jr., 1962. Circulation of the cerebrospinal fluid. Demonstration of the choroid plexuses as the generator of the force for flow of fluid and ventricular enlargement. J. Neurosurg. 19:405-413.

Bhatt, D.K., Gupta, S., Olesen, J., Jansen-Olesen, I., 2014. PACAP-38 infusion causes sustained vasodilation of the middle meningeal artery in the rat: possible involvement of mast cells. Cephalalgia 34, 877-886.

Biedl, A., Kraus, R., 1898. Über eine bisher unbekannte toxische Wirkung der Gallensaure auf das Zentralnervensystem. 19,1185 -1200.

Bifari, F., Decimo, I., Chiamulera, C., Bersan, E., Malpeli, G., Johansson, J., Lisi, V., Bonetti, B., Fumagalli, G., Pizzolo, G., Krampera, M., 2009. Novel stem/progenitor cells with neuronal differentiation potential reside in the leptomeningeal niche. J. Cell. Mol. Med. 13, 31953208.

Bilston, L.E., Fletcher, D.F., Brodbelt, A.R., Stoodley, M.A., 2003. Arterial pulsation-driven cerebrospinal fluid flow in the perivascular space: a computational model. Comput. Methods Biomech. Biomed. Engin. 6, 235-241.

Bito, L., Davson, H., Levin, E., Murray, M., Snider, N., 1966. The concentrations of free amino acids and other electrolytes in cerebrospinal fluid, in vivo dialysate of brain, and blood plasma of the dog. J. Neurochem. 13, 1057-1067.

Bito, L.Z., Davson, H., 1966. Local variations in cerebrospinal fluid composition and its relationship to the composition of the extracellular fluid of the cortex. Exp. Neurol. 14, 264280.

Blinder, P., Tsai, P.S., Kaufhold, J.P., Knutsen, P.M., Suhl, H., Kleinfeld, D., 2013. The cortical angiome: an interconnected vascular network with noncolumnar patterns of blood flow. Nat. Neurosci. 16, 889-897.

Boehm, R., 1869. Experimentelle Studien über die Dura mater des Menschen und der Säugetiere. Arch. Pathol. Anat. Physiol. (Virchows Archiv) 47, 218-234.

Bolay, H., Reuter, U., Dunn, A.K., Huang, Z., Boas, D.A., Moskowitz, M.A., 2002. Intrinsic brain activity triggers trigeminal meningeal afferents in a migraine model. Nat. Med. 8, 136-142.

Boron, W.F., 2010. Sharpey-Schafer lecture: gas channels. Exp. Physiol. 95, 1107-1130.

Bouffard, G., 1906. Injection des couleurs de benzidine aux animaux normaux. Ann. Inst. Pasteur 20, 539 - 548.

Boulton, M., Flessner, M., Armstrong, D., Mohamed, R., Hay, J., Johnston, M., 1999. Contribution of extracranial lymphatics and arachnoid villi to the clearance of a CSF tracer in the rat. Am. J. Physiol. 276, R818-823.

Bove, G.M., Moskowitz, M.A., 1997. Primary afferent neurons innervating guinea pig dura. J. Neurophysiol. 77, 299-308.

Boyer, N., Dallel, R., Artola, A., Monconduit, L., 2014. General trigeminospinal central sensitization and impaired descending pain inhibitory controls contribute to migraine progression. Pain 155, 1196-1205.

Bradbury, M.W., Cserr, H.F., Westrop, R.J., 1981. Drainage of cerebral interstitial fluid into deep cervical lymph of the rabbit. Am. J. Physiol. 240, F329-336.

Bradbury, M.W., Westrop, R.J., 1983. Factors influencing exit of substances from cerebrospinal fluid into deep cervical lymph of the rabbit. J. Physiol. 339, 519-534.

Brendecke, S.M., Prinz, M., 2015. Do not judge a cell by its cover-diversity of CNS resident, adjoining and infiltrating myeloid cells in inflammation. Semin. Immunopathol. 37, 591605 .

Brightman, M.W., 1977. Morphology of blood-brain interfaces. Exp. Eye Res. 25 Suppl., 1-25.

Brightman, M.W., Reese, T.S., 1969. Junctions between intimately apposed cell membranes in the vertebrate brain. J. Cell Biol. 40, 648-677. 
Broman, T., 1940. Über die Blut-Hirnschranke, ihre Bedeutung und ihre Beziehungen zur BlutLiquorschranke. Arch. Psychiat. 112, 309-326.

Broman, T. (1949) The permeability of the cerebrospinal vessels in normal and pathological conditions. Copenhagen: Ejnar Munksgaard.

Bruce, A.N., 1913. Vaso-dilator axon-reflexes. Quart. J. Exp. Physiol. 6, 339 - 354.

Brunori, A., Vagnozzi, R., Giuffre, R., 1993. Antonio Pacchioni (1665-1726): early studies of the dura mater. J. Neurosurg. 78, 515-518.

Bruttger, J., Karram, K., Wortge, S., Regen, T., Marini, F., Hoppmann, N., Klein, M., Blank, T., Yona, S., Wolf, Y., Mack, M., Pinteaux, E., Muller, W., Zipp, F., Binder, H., Bopp, T., Prinz, M., Jung, S., Waisman, A., 2015. Genetic Cell Ablation Reveals Clusters of Local Self-Renewing Microglia in the Mammalian Central Nervous System. Immunity 43, 92-106.

Bucchieri, F., Farina, F., Zummo, G., Cappello, F., 2015. Lymphatic vessels of the dura mater: a new discovery? J. Anat. 227, 702-703.

Bulloch, K., Miller, M.M., Gal-Toth, J., Milner, T.A., Gottfried-Blackmore, A., Waters, E.M., Kaunzner, U.W., Liu, K., Lindquist, R., Nussenzweig, M.C., Steinman, R.M., McEwen, B.S., 2008. CD11c/EYFP transgene illuminates a discrete network of dendritic cells within the embryonic, neonatal, adult, and injured mouse brain. J. Comp. Neurol. 508, 687-710.

Burstein, R., Noseda, R., Borsook, D., 2015. Migraine: multiple processes, complex pathophysiology. J. Neurosci. 35, 6619-6629.

Butler, A.B., Mann, J.D., Bass, N.H., 1975. Identification of the major site for cerebrospinal fluid efflux in the albino rat. Anat. Rec. 181, 323.

Cabanac, M., Brinnel, H., 1985. Blood flow in the emissary veins of the human head during hyperthermia. Eur. J. Appl. Physiol. Occup. Physiol. 54, 172-176.

Caesar, K., Hashemi, P., Douhou, A., Bonvento, G., Boutelle, M.G., Walls, A.B., Lauritzen, M., 2008. Glutamate receptor-dependent increments in lactate, glucose and oxygen metabolism evoked in rat cerebellum in vivo. J. Physiol. 586, 1337-1349.

Cammermeyer, J., 1971. Median and caudal apertures in the roof of the fourth ventricle in rodents and primates. J. Comp. Neurol. 141, 499-512.

Campagnola, P.J., Loew, L.M., 2003. Second-harmonic imaging microscopy for visualizing biomolecular arrays in cells, tissues and organisms. Nat. Biotechnol. 21, 1356-1360.

Cao, Y., Welch, K.M., Aurora, S., Vikingstad, E.M., 1999. Functional MRI-BOLD of visually triggered headache in patients with migraine. Arch. Neurol. 56, 548-554.

Carare, R.O., Bernardes-Silva, M., Newman, T.A., Page, A.M., Nicoll, J.A., Perry, V.H., Weller, R.O., 2008. Solutes, but not cells, drain from the brain parenchyma along basement membranes of capillaries and arteries: significance for cerebral amyloid angiopathy and neuroimmunology. Neuropathol. Appl. Neurobiol. 34, 131-144.

Carare, R.O., Hawkes, C.A., Jeffrey, M., Kalaria, R.N., Weller, R.O., 2013. Review: cerebral amyloid angiopathy, prion angiopathy, CADASIL and the spectrum of protein elimination failure angiopathies (PEFA) in neurodegenerative disease with a focus on therapy. Neuropathol. Appl. Neurobiol. 39, 593-611.

Carare, R.O., Hawkes C.A., Weller R.O., 2014. Afferent and efferent immunological pathways of the brain. Anatomy, function and failure. Brain Behav. Immun. 36, 9-14.

Cardona, A.E., Huang, D., Sasse, M.E., Ransohoff, R.M., 2006a. Isolation of murine microglial cells for RNA analysis or flow cytometry. Nat. Protoc. 1, 1947-1951.

Cardona, A.E., Pioro, E.P., Sasse, M.E., Kostenko, V., Cardona, S.M., Dijkstra, I.M., Huang, D., Kidd, G., Dombrowski, S., Dutta, R., Lee, J.C., Cook, D.N., Jung, S., Lira, S.A., Littman, D.R., Ransohoff, R.M., 2006b. Control of microglial neurotoxicity by the fractalkine receptor. Nat. Neurosci. 9, 917-924.

Carp, R.I., Davidson, A.L., Merz, P.A., 1971. A method for obtaining cerebrospinal fluid from mice. Res. Vet. Sci. 12, 499. 
Carrithers, M.D., Visintin, I., Viret, C., Janeway, C.S., Jr., 2002. Role of genetic background in P selectin-dependent immune surveillance of the central nervous system. J. Neuroimmunol. 129, 51-57.

Cathelin, F., 1903. La circulation du liquide céphalo-rachidien. CR. Soc. Biol. 55, 1167-1169.

Chen, L., Beckett, A., Verma, A., Feinberg, D.A., 2015. Dynamics of respiratory and cardiac CSF motion revealed with real-time simultaneous multi-slice EPI velocity phase contrast imaging. Neuroimage 122, 281-287.

Chinnery, H.R., Ruitenberg, M.J., McMenamin, P.G., 2010. Novel characterization of monocytederived cell populations in the meninges and choroid plexus and their rates of replenishment in bone marrow chimeric mice. J. Neuropathol. Exp. Neurol. 69, 896-909.

Chorobski, J., Penfield, W., 1932. Cerebral vasodilator nerves and their pathway from the medulla oblongata. Arch. Neurol. 28, 1257-1289.

Christodoulides, M. (2013) Meningitis: Cellular and Molecular Basis: Advances in Molecular and Cellular Microbiology, 26. CAB International.

Christy, A.L., Walker, M.E., Hessner, M.J., Brown, M.A., 2013. Mast cell activation and neutrophil recruitment promotes early and robust inflammation in the meninges in EAE. J. Autoimmun. 42, 50-61.

Coles, J.A., Myburgh, E., Ritchie, R., Hamilton, A., Rodgers, J., Mottram, J.C., Barrett, M.P., Brewer, J.M., 2015. Intravital imaging of a massive lymphocyte response in the cortical dura of mice after peripheral infection by trypanosomes. PLoS Negl. Trop. Dis. 9, e0003714. doi:10.1371/journal.pntd.0003714.

Coles, J.A., Poulain, D.A., 1991. Extracellular $\mathrm{K}^{+}$in the supraoptic nucleus of the rat during reflex bursting activity by oxytocin neurones. J. Physiol. 43, 383-409.

Coles, J.A., Stewart-Hutchinson, P.J., Myburgh, E., Brewer, J.M., 2017. The mouse cortical meninges are the site of immune responses to many different pathogens, and are accessible to intravital imaging. Methods. doi: org/10.1016/j.ymeth.2017.03.020

Coloma, M., Schaffer, J.D., Carare, R.O., Chiarot, P.R., Huang, P., 2016. Pulsations with reflected boundary waves: a hydrodynamic reverse transport mechanism for perivascular drainage in the brain. J. Math. Biol. 73, 469-490.

Constantinescu, C.S., Farooqi, N., O'Brien, K., Gran, B., 2011. Experimental autoimmune encephalomyelitis (EAE) as a model for multiple sclerosis (MS). Br. J. Pharmacol. 164, 1079-1106.

Coquery, N., Francois, O., Lemasson, B., Debacker, C., Farion, R., Remy, C., Barbier, E.L., 2014. Microvascular MRI and unsupervised clustering yields histology-resembling images in two rat models of glioma. J. Cereb. Blood Flow Metab. 34, 1354-1362.

Couly, G.F., Le Douarin, N.M., 1987. Mapping of the early neural primordium in quail-chick chimeras. II. The prosencephalic neural plate and neural folds: implications for the genesis of cephalic human congenital abnormalities. Dev. Biol. 120, 198-214.

Cox, S.B., Woolsey, T.A., Rovainen, C.M., 1993. Localized dynamic changes in cortical blood flow with whisker stimulation corresponds to matched vascular and neuronal architecture of rat barrels. J. Cereb. Blood Flow Metab. 13, 899-913.

Cragg, B., 1979. Brain extracellular space fixed for electron microscopy. Neurosci. Lett. 15, 301306.

Csati, A., Tajti, J., Kuris, A., Tuka, B., Edvinsson, L., Warfvinge, K., 2012. Distribution of vasoactive intestinal peptide, pituitary adenylate cyclase-activating peptide, nitric oxide synthase, and their receptors in human and rat sphenopalatine ganglion. Neuroscience 202, $158-168$.

Cserr, H.F., Bundgaard, M., 1984. Blood-brain interfaces in vertebrates: a comparative approach. Am. J. Physiol. 246, R277-288.

Cserr, H.F., Cooper, D.N., Milhorat, T.H., 1977. Flow of cerebral interstitial fluid as indicated by the removal of extracellular markers from rat caudate nucleus. Exp. Eye Res. 25 Suppl., 461-473. 
Cserr, H.F., Cooper, D.N., Suri, P.K., Patlak, C.S., 1981. Efflux of radiolabeled polyethylene glycols and albumin from rat brain. Am. J. Physiol. 240, F319-328.

Cserr, H.F., Harling-Berg, C.J., Knopf, P.M., 1992. Drainage of brain extracellular fluid into blood and deep cervical lymph and its immunological significance. Brain Pathol. 2, 269-276.

Cserr, H.F., Ostrach, L.H., 1974. Bulk flow of interstitial fluid after intracranial injection of blue dextran 2000. Exp. Neurol. 45, 50-60.

Cui, Y., Toyoda, H., Sako, T., Onoe, K., Hayashinaka, E., Wada, Y., Yokoyama, C., Onoe, H., Kataoka, Y., Watanabe, Y., 2015. A voxel-based analysis of brain activity in high-order trigeminal pathway in the rat induced by cortical spreading depression. Neuroimage 108, $17-22$.

Cushing, H., 1914. Studies on the Cerebro-Spinal Fluid : I. Introduction. J. Med. Res. 31, 1-19.

Cushing, H., 1925. Cameron lectures. 1. The third circulation and its channels. Lancet 206, 851857.

Dahl, E., 1973. The innervation of the cerebral arteries. J. Anat. 115, 53-63.

Dai, Y., 2016. TRPs and pain. Semin. Immunopathol. 38, 277-291.

Daley, J.M., Thomay, A.A., Connolly, M.D., Reichner, J.S., Albina, J.E., 2008. Use of Ly6Gspecific monoclonal antibody to deplete neutrophils in mice. J. Leukoc. Biol. 83, 64-70.

Dandy, W.E., Blackfan, K.D., 1913. An experimental and clinical study of internal hydrocephalus. JAMA 61, 2216-2217.

Davson, H., 1972. Dynamic aspects of cerebrospinal fluid. Dev. Med. Child. Neurol. Suppl. 27, 116.

Davson, H., 1976. Review lecture. The blood-brain barrier. J. Physiol. 255, 1-28.

Davson, H., Hollingsworth, G., Segal, M.B., 1970. The mechanism of drainage of the cerebrospinal fluid. Brain 93, 665-678.

Davson, H., Segal M.B. (1996) Physiology of the CSF and Blood-Brain Barriers. Boca Raton, FL.: CRC Press.

De Bock, M., Wang, N., Decrock, E., Bol M., Gadicherla, A.K., Culot, M., Cecchelli, R., Bultynck, G., Leybaert, L., 2013. Endothelial calcium dynamics, connexin channels and blood-brain barrier function. Prog. Neurobiol. 108, 1-20.

Denk, W., Strickler, J.H., Webb, W.W., 1990. Two-photon laser scanning fluorescence microscopy. Science 248, 73-76.

Denk, W., Svoboda, K., 1997. Photon upmanship: why multiphoton imaging is more than a gimmick. Neuron 18, 351-357.

Derecki, N.C., Cardani, A.N., Yang, C.H., Quinnies, K.M., Crihfield, A., Lynch, K.R., Kipnis, J., 2010. Regulation of learning and memory by meningeal immunity: a key role for IL-4. J. Exp. Med. 207, 1067-1080.

Devesa, I., Ferrandiz-Huertas, C., Mathivanan, S., Wolf, C., Lujan, R., Changeux, J.P., FerrerMontiel, A., 2014. $\alpha$ CGRP is essential for algesic exocytotic mobilization of TRPV1 channels in peptidergic nociceptors. Proc. Natl Acad. Sci. U S A 111, 18345-18350.

Devorak, J., Torres-Platas, S.G., Davoli, M.A., Prud'homme, J., Turecki, G., Mechawar, N., 2015. Cellular and Molecular Inflammatory Profile of the Choroid Plexus in Depression and Suicide. Front. Psychiatry 6, 138.

Dietzel, I., Heinemann, U., Hofmeier, G., Lux, H.D., 1980. Transient changes in the size of the extracellular space in the sensorimotor cortex of cats in relation to stimulus-induced changes in potassium concentration. Exp. Brain Res. 40, 432-439.

Dimitriadou, V., Buzzi, M.G., Moskowitz, M.A., Theoharides, T.C., 1991. Trigeminal sensory fiber stimulation induces morphological changes reflecting secretion in rat dura mater mast cells. Neuroscience 44, 97-112.

Dimitriadou, V., Rouleau, A., Trung Tuong, M.D., Newlands, G.J., Miller, H.R., Luffau, G., Schwartz, J.C., Garbarg, M., 1997. Functional relationships between sensory nerve fibers and mast cells of dura mater in normal and inflammatory conditions. Neuroscience 77, 829839. 
Dimlich, R.V., Keller, J.T., Strauss, T.A., Fritts, M.J., 1991. Linear arrays of homogeneous mast cells in the dura mater of the rat. J. Neurocytol. 20, 485-503.

Djukic, M., Mildner, A., Schmidt, H., Czesnik, D., Bruck, W., Priller, J., Nau, R., Prinz, M., 2006. Circulating monocytes engraft in the brain, differentiate into microglia and contribute to the pathology following meningitis in mice. Brain 129, 2394-2403.

Dorr, A., Sled, J.G., Kabani, N., 2007. Three-dimensional cerebral vasculature of the CBA mouse brain: a magnetic resonance imaging and micro computed tomography study. Neuroimage 35, 1409-1423.

Dreha-Kulaczewski, S., Joseph, A.A., Merboldt, K.D., Ludwig, H.C., Gartner, J., Frahm, J., 2015. Inspiration is the major regulator of human CSF flow. J. Neurosci. 35, 2485-2491.

Dreha-Kulaczewski, S., Joseph, A.A., Merboldt, K.D., Ludwig, H.C., Gartner, J., Frahm, J., 2017. Identification of the upward movement of human CSF in vivo and its relation to the brain venous system. J. Neurosci. 37, 2395-2402.

Drew, P.J., Shih, A.Y., Driscoll, J.D., Knutsen, P.M., Blinder, P., Davalos, D., Akassoglou, K., Tsai, P.S., Kleinfeld, D., 2010. Chronic optical access through a polished and reinforced thinned skull. Nat. Methods 7, 981-984.

Drobizhev, M., Makarov, N.S., Tillo, S.E., Hughes, T.E., Rebane, A., 2011. Two-photon absorption properties of fluorescent proteins. Nat. Methods 8, 393-399.

Dropp, J.J., 1972. Mast cells in the central nervous system of several rodents. Anat. Rec. 174, $227-$ 237.

Drummond, P.D., 1991. Cervical sympathetic deficit in unilateral migraine headache. Headache 31, 669-672.

Du Boulay, G., 1972. Specialisation broadens the view. The significance of a C.S.F. pulse. Clin. Radiol. 23, 401-409.

Dunker, R.O., Harris, A.B., Jenkins, D.P., 1976. Kinetics of horseradish peroxidase migration through cerebral cortex. Brain Res. 118, 199-217.

Dux, M., Santha, P., Jancso, G., 2012. The role of chemosensitive afferent nerves and TRP ion channels in the pathomechanism of headaches. Pflugers Arch. 464, 239-248.

Ebersberger, A., Averbeck, B., Messlinger, K., Reeh, P.W., 1999a. Release of substance P, calcitonin gene-related peptide and prostaglandin E2 from rat dura mater encephali following electrical and chemical stimulation in vitro. Neuroscience 89, 901-907.

Ebersberger, A., Handwerker, H.O., Reeh, P.W., 1999b. Nociceptive neurons in the rat caudal trigeminal nucleus respond to blood plasma perfusion of the subarachnoid space: the involvement of complement. Pain 81, 283-288.

Ebersberger, A., Ringkamp, M., Reeh, P.W., Handwerker, H.O., 1997. Recordings from brain stem neurons responding to chemical stimulation of the subarachnoid space. J. Neurophysiol. 77, 3122-3133.

Ebersberger, A., Takac, H., Richter, F., Schaible, H.G., 2006. Effect of sympathetic and parasympathetic mediators on the release of calcitonin gene-related peptide and prostaglandin $\mathrm{E}$ from rat dura mater, in vitro. Cephalalgia 26, 282-289.

Edvinsson, L., Brodin, E., Jansen, I., Uddman, R., 1988. Neurokinin A in cerebral vessels: characterization, localization and effects in vitro. Regul. Pept. 20,181-197.

Edvinsson, L., Copeland, J.R., Emson, P.C., McCulloch, J., Uddman, R., 1987. Nerve fibers containing neuropeptide $\mathrm{Y}$ in the cerebrovascular bed: immunocytochemistry, radioimmunoassay, and vasomotor effects. J. Cereb. Blood Flow Metab. 7, 45-57.

Edvinsson, L., Gulbenkian, S., Barroso, C.P., Cunha e Sa, M., Polak, J.M., Mortensen, A., Jorgensen, L., Jansen-Olesen, I., 1998a. Innervation of the human middle meningeal artery: immunohistochemistry, ultrastructure, and role of endothelium for vasomotility. Peptides $19,1213-1225$.

Edvinsson, L., McCulloch, J., Uddman, R., 1981. Substance P: immunohistochemical localization and effect upon cat pial arteries in vitro and in situ. J. Physiol. 318, 251-258. 
Edvinsson, L., Mulder, H., Goadsby, P.J., Uddman, R., 1998b. Calcitonin gene-related peptide and nitric oxide in the trigeminal ganglion: cerebral vasodilatation from trigeminal nerve stimulation involves mainly calcitonin gene-related peptide. J. Auton. Nerv. Syst. 70, 15-22.

Edvinsson, L., Nielsen, K.C., Owman, C., Sporrong, B., 1972. Cholinergic mechanisms in pial vessels. Histochemistry, electron microscopy and pharmacology. Z. Zellforsch. Mikrosk. Anat. 134, 311-325.

Edvinsson, L., Uddman, R., 1981. Adrenergic, cholinergic and peptidergic nerve fibres in dura mater - involvement in headache? Cephalalgia 1, 175-179.

Edvinsson, L., Uddman, R., 2005. Neurobiology in primary headaches. Brain Res. Brain Res. Rev. 48, 438-456.

Eftekhari, S., Salvatore, C.A., Johansson, S., Chen, T.B., Zeng, Z., Edvinsson, L., 2015. Localization of CGRP, CGRP receptor, PACAP and glutamate in trigeminal ganglion. Relation to the blood-brain barrier. Brain Res. 1600, 93-109.

Eftekhari, S., Warfvinge, K., Blixt, F.W., Edvinsson, L., 2013. Differentiation of nerve fibers storing CGRP and CGRP receptors in the peripheral trigeminovascular system. J. Pain 14, 1289-1303.

Ehrlich, P., 1877. Beiträge zur Kenntniss der Anilinfärbungen und ihrer Verwendung in der mikroskopischen Technik. Archiv für Mikroskopische Anatomie 13, 263-277.

Eichling, J.O., Raichle, M.E., Grubb, R.L., Jr., Ter-Pogossian, M.M., 1974. Evidence of the limitations of water as a freely diffusible tracer in brain of the rhesus monkey. Circ. Res. 35, 358-364.

Elmquist, J.K., Breder, C.D., Sherin, J.E., Scammell, T.E., Hickey, W.F., Dewitt, D., Saper, C.B., 1997. Intravenous lipopolysaccharide induces cyclooxygenase 2-like immunoreactivity in rat brain perivascular microglia and meningeal macrophages. J. Comp. Neurol. 381, 119129.

Engelhardt, B., Wolburg-Buchholz, K., Wolburg, H., 2001. Involvement of the choroid plexus in central nervous system inflammation. Microsc. Res. Tech. 52, 112-129.

Engelhardt, B., Vajkoczy, P., Weller, R.O., 2017. The movers and shapers in immune privilege of the CNS. Nat. Immunol. 18, 123-131.

Engstrom, L., Ruud, J., Eskilsson, A., Larsson, A., Mackerlova, L., Kugelberg, U., Qian, H., Vasilache, A.M., Larsson, P., Engblom, D., Sigvardsson, M., Jonsson, J.I., Blomqvist, A., 2012. Lipopolysaccharide-induced fever depends on prostaglandin E2 production specifically in brain endothelial cells. Endocrinology 153, 4849-4861.

Enzmann, G., Mysiorek, C., Gorina, R., Cheng, Y.J., Ghavampour, S., Hannocks, M.J., Prinz, V., Dirnagl, U., Endres, M., Prinz, M., Beschorner, R., Harter, P.N., Mittelbronn, M., Engelhardt, B., Sorokin, L., 2013. The neurovascular unit as a selective barrier to polymorphonuclear granulocyte (PMN) infiltration into the brain after ischemic injury. Acta Neuropathol. 125, 395-412.

Enzmann, D.R., Pelc, N.J., 1993. Cerebrospinal fluid flow measured by phase-contrast cine MR. AJNR Am. J. Neuroradiol. 14, 1301-1307; discussion 1309-1310.

Feinberg, D.A., Mark A.S., 1987. Human brain motion and cerebrospinal fluid circulation demonstrated with MR velocity imaging. Radiology 163, 793-799.

Eyre, J.A., Essex, T.J., Flecknell, P.A., Bartholomew, P.H., Sinclair, J.I., 1988. A comparison of measurements of cerebral blood flow in the rabbit using laser Doppler spectroscopy and radionuclide labelled microspheres. Clin. Phys. Physiol. Meas. 9:65-74.

Falck, B., McHedlishvili, G.I., Owman, C., 1965. Histochemical demonstration of adrenergic nerves in cortex-pia of rabbit. Acta Pharmacol. Toxicol. (Copenh) 23, 133-142.

Falk, D., 1986. Evolution of cranial blood drainage in hominids: enlarged occipital/marginal sinuses and emissary foramina. Am. J. Phys. Anthropol. 70, 311-324.

Faraci, F.M., Kadel, K.A., Heistad, D.D., 1989. Vascular responses of dura mater. Am. J. Physiol. 257, H157-161.

Fay, T., 1935. The mechanism of headache. Trans. Am. Neurol. Assoc. 62, 74-77. 
Feng, C.Y., Wiggins, L.M., von Bartheld, C.S., 2011. The locus ceruleus responds to signaling molecules obtained from the CSF by transfer through tanycytes. J. Neurosc. 31, 9147-9158.

Fercher, A.F., Briers, J.D., 1981. Flow visualization by means of single-exposure speckle photography. Opt. Comm. 37, 326-330.

Fernandez-Klett, F., Offenhauser, N., Dirnagl, U., Priller, J., Lindauer, U., 2010. Pericytes in capillaries are contractile in vivo, but arterioles mediate functional hyperemia in the mouse brain. Proc. Natl Acad. Sci. U S A 107, 22290-22295.

Fieschi, C., Lenzi, G.L., Zanette, E., Orzi, F., Passero, S., 1980. Effects on EEG of the osmotic opening of the blood-brain barrier in rats. Life Sci, 27, 239-243.

Fischkoff, S., Vanderkooi, J.M., 1975. Oxygen diffusion in biological and artificial membranes determined by the fluorochrome pyrene. J. Gen. Physiol. 65, 663-676.

Fisher, L.A., Kikkawa, D.O., Rivier, J.E., Amara, S.G., Evans, R.M., Rosenfeld, M.G., Vale, W.W., Brown, M.R., 1983. Stimulation of noradrenergic sympathetic outflow by calcitonin generelated peptide. Nature 305, 534-536.

Flexner, L.B., 1933. Some problems of the origin, circulation and absorption of the cerebrospinal fluid. Quart. Rev. Biol. 8, 397-422.

Flexner, L.B., Winters, H., 1932. The rate of formation of cerebrospinal fluid in etherized cats. Am. J. Physiol. 101, 697 - 710.

Flugel, A., Bradl, M., Kreutzberg, G.W., Graeber, M.B., 2001. Transformation of donor-derived bone marrow precursors into host microglia during autoimmune CNS inflammation and during the retrograde response to axotomy. J. Neurosci. Res. 66, 74-82.

Földi, M., Csanda, E., Obál, F., Madarász, I., Szeghy, G., Zoltán, Ö.T., 1963. Über Wirkungen der Unterbindung der Lymphgefässe und Lymphknoten des Halses auf das Zentralnervensystem im Tierversuch I. Mitteilung. Z. ges. exp. Med. 137, 483-510.

Földi, M., Csillik, B., Zoltan, O.T., 1968. Lymphatic drainage of the brain. Experientia 24, 12831287.

Földi, M., Gellért, A., Kozma, M., Poberai, M., Zoltán, Ö.T., Csanda, E., 1966. New contributions to the anatomical connections of the brain and the lymphatic system. Acta Anat. (Basel) 64, 498-505.

Forbes, H.S., 1928. The cerebral circulation: I. Observation and measurement of pial vessels. Arch. Neurol. 19, 751- 761.

Forbes, H.S., Cobb, S.S., 1938. Vasomotor control of cerebral vessels. Brain 61, 221-233.

Forbes, H.S., Wolff, H.G., 1928. Cerebral Circulation. III. The vasomoter control of cerebral vessels. Arch. Neurol. 19, 1057-1086.

Frederickson, R.G., 1991. The subdural space interpreted as a cellular layer of meninges. Anat. Rec. 230, 38-51.

Frederickson, R.G., Low, F.N., 1969. Blood vessels and tissue space associated with the brain of the rat. Am. J .Anat. 125, 123-145.

Frederiks, J.A., Koehler, P.J., 1997. The first lumbar puncture. J. Hist. Neurosci. 6, 147-153.

Fricke B., Andres, K.H., Von Duering, M., 2001. Nerve fibers innervating the cranial and spinal meninges: morphology of nerve fiber terminals and their structural integration. Microsc. Res. Tech. 53, 96-105.

Fricke, B., von Duering, M., Andres, K.H., 1997. Topography and immunocytochemical characterization of nerve fibers in the leptomeningeal compartments of the rat. A light- and electron-microscopical study. Cell Tissue Res. 287, 11-22.

Fumagalli, S., Coles, J.A., Ejlerskov, P., Ortolano, F., Bushell, T.J., Brewer, J.M., De Simoni, M.G., Dever, G., Garside, P., Maffia, P., Carswell, H.V., 2011. In vivo real-time multiphoton imaging of T lymphocytes in the mouse brain after experimental stroke. Stroke 42, 1429-1436.

Furukawa, M., Shimoda, H., Kajiwara, T., Kato, S., Yanagisawa, S., 2008. Topographic study on nerve-associated lymphatic vessels in the murine craniofacial region by immunohistochemistry and electron microscopy. Biomed. Res. 29, 289-296. 
Gabbiani, G., Badonnel, M.C., Majno, G., 1970. Intra-arterial injections of histamine, serotonin, or bradykinin: a topographic study of vascular leakage. Proc. Soc. Exp. Biol. Med. 135, 447452.

Galea, I., Bechmann, I., Perry, V.H., 2007. What is immune privilege (not)? Trends Immunol. 28, 12-18.

Garside, P., Brewer, J., 2010. In vivo imaging of infection immunology--4I's! Semin. Immunopathol. 32, 289-296.

Gärtner, W., 1927. Die Blut-Liquorschranke. Zschr. f. Biol. 86, 115.

Gerber, J., Raivich, G., Wellmer, A., Noeske, C., Kunst, T., Werner, A., Bruck, W., Nau, R., 2001. A mouse model of Streptococcus pneumoniae meningitis mimicking several features of human disease. Acta Neuropathol. 101, 499-508.

Ghanavati, S., Lerch, J.P., Sled, J.G., 2014. Automatic anatomical labeling of the complete cerebral vasculature in mouse models. Neuroimage 95, 117-128.

Ghersi-Egea, J.F., Finnegan, W., Chen, J.L., Fenstermacher, J.D., 1996. Rapid distribution of intraventricularly administered sucrose into cerebrospinal fluid cisterns via subarachnoid velae in rat. Neuroscience 75, 1271-1288.

Gibbins, I.L., Furness, J.B., Costa, M., MacIntyre, I., Hillyard, C.J., Girgis, S., 1985. Colocalization of calcitonin gene-related peptide-like immunoreactivity with substance $\mathrm{P}$ in cutaneous, vascular and visceral sensory neurons of guinea pigs. Neurosci. Lett. 57, 125 130.

Gibbins, I.L., Morris, J.L., 1988. Co-existence of immunoreactivity to neuropeptide Y and vasoactive intestinal peptide in non-noradrenergic axons innervating guinea pig cerebral arteries after sympathectomy. Brain Res. 444, 402-406.

Gideon, P., Stahlberg, F., Thomsen, C., Gjerris, F., Sorensen, P.S., Henriksen, O., 1994. Cerebrospinal fluid flow and production in patients with normal pressure hydrocephalus studied by MRI. Neuroradiology 36, 210-215.

Ginhoux, F., Greter, M., Leboeuf, M., Nandi, S., See, P., Gokhan, S., Mehler, M.F., Conway, S.J., Ng, L.G., Stanley, E.R., Samokhvalov, I.M., Merad, M., 2010. Fate mapping analysis reveals that adult microglia derive from primitive macrophages. Science 330, 841-845.

Goldmann, E.E., 1909. Die äussere und innere Sekretion des gesunden und kranken Organismus im Lichte der "vitalen Färbung". Teil 1. Beitr. z. Klin. Chir. 64, 192-265.

Goldmann, E.E., 1913. Vitalfärbung am Zentralnervensystem. Abh. Königlich. Preuss. Akad. Wiss. Physikalisch-Mathematische Klasse Abh. 1, 1-60.

Goldschmidt, R.C., Hough, L.B., Glick, S.D., Padawer, J., 1984. Mast cells in rat thalamus: nuclear localization, sex difference and left-right asymmetry. Brain Res. 323, 209-217.

Golomb, J., Wisoff, J., Miller, D.C., Boksay, I., Kluger, A., Weiner, H., Salton, J., Graves, W., 2000. Alzheimer's disease comorbidity in normal pressure hydrocephalus: prevalence and shunt response. J. Neurol. Neurosurg. Psychiatry 68, 778-781.

Gomez-Nicola, D., Schetters, S.T., Perry, V.H., 2014. Differential role of CCR2 in the dynamics of microglia and perivascular macrophages during prion disease. Glia 62, 1041-1052.

Göppert-Mayer, M., 1931. Über Elementarakte mit zwei Quantensprüngen. Annalen der Physik 401, 273-294.

Gordon, G.R., Mulligan, S.J., MacVicar, B.A., 2007. Astrocyte control of the cerebrovasculature. Glia 55, 1214-1221.

Gordon, L.B., Knopf, P.M., Cserr, H.F., 1992. Ovalbumin is more immunogenic when introduced into brain or cerebrospinal fluid than into extracerebral sites. J. Neuroimmunol. 40, 81-87.

Gotoh, F., Komatsumoto, S., Araki, N., Gomi, S., 1984. Noradrenergic nervous activity in migraine. Arch. Neurol. 41, 951-955.

Graesser, D., Solowiej, A., Bruckner, M., Osterweil, E., Juedes, A., Davis, S., Ruddle, N.H., Engelhardt, B., Madri, J.A., 2002. Altered vascular permeability and early onset of experimental autoimmune encephalomyelitis in PECAM-1-deficient mice. J. Clin. Invest. 109, 383-392. 
Greenhill, S.D., Ranson, A., Fox, K., 2015. Hebbian and homeostatic plasticity mechanisms in regular spiking and intrinsic bursting cells of cortical layer 5. Neuron 88, 539-552.

Greter, M., Lelios, I., Croxford, A.L., 2015. Microglia Versus Myeloid Cell Nomenclature during Brain Inflammation. Front. Immunol. 6, 249.

Grisold, W., Grisold, A., 2014. Cancer around the brain. Neurooncol. Pract. 1, 13-21.

Groeschel, S., Chong, W.K., Surtees, R., Hanefeld, F., 2006. Virchow-Robin spaces on magnetic resonance images: normative data, their dilatation, and a review of the literature. Neuroradiology 48, 745-754.

Grutzendler, J., Kasthuri, N., Gan, W.B., 2002. Long-term dendritic spine stability in the adult cortex. Nature 420, 812-816.

Guild, S.J., McBryde, F.D., Malpas, S.C., 2015. Recording of intracranial pressure in conscious rats via telemetry. J. Appl. Physiol. 119, 576-581.

Guilliams, M., Ginhoux, F., Jakubzick, C., Naik, S.H., Onai, N., Schraml, B.U., Segura, E., Tussiwand, R., Yona, S., 2014. Dendritic cells, monocytes and macrophages: a unified nomenclature based on ontogeny. Nat. Rev. Immunol. 14, 571-578.

Hadaczek, P., Yamashita, Y., Mirek, H., Tamas, L., Bohn, M.C., Noble, C., Park, J.W., Bankiewicz, K., 2006. The "perivascular pump" driven by arterial pulsation is a powerful mechanism for the distribution of therapeutic molecules within the brain. Mol. Ther. 14, 6978.

Hadjikhani, N., Sanchez Del Rio, M., Wu, O., Schwartz, D., Bakker, D., Fischl, B., Kwong, K.K., Cutrer, F.M., Rosen, B.R., Tootell, R.B., Sorensen, A.G., Moskowitz, M.A., 2001.

Mechanisms of migraine aura revealed by functional MRI in human visual cortex. Proc. Natl Acad. Sci. U S A 98, 4687-4692.

Haines, D.E., 1991. On the question of a subdural space. Anat. Rec. 230, 3-21.

Haines, D.E., Harkey, H.L., al-Mefty, O., 1993. The "subdural" space: a new look at an outdated concept. Neurosurgery 32, 111-120.

Han, M.E., Kim, H.J., Lee, Y.S., Kim, D.H., Choi, J.T., Pan, C.S., Yoon, S., Baek, S.Y., Kim, B.S., Kim, J.B., Oh, S.O., 2009. Regulation of cerebrospinal fluid production by caffeine consumption. BMC Neurosci. 10, 110.

Hansen, J.M., Ashina, M., 2014. Calcitonin gene-related peptide and migraine with aura: A systematic review. Cephalalgia 34, 695-707.

Harper, A.M., Bell, R.A., 1963. The effect of metabolic acidosis and alkalosis on the blood flow through the cerebral ortex. J. Neurol. Neurosurg. Psychiatry. 26, 341-344.

Hawkes, C.A., Jayakody, N., Johnston, D.A., Bechmann, I., Carare, R.O., 2014. Failure of perivascular drainage of beta-amyloid in cerebral amyloid angiopathy. Brain Pathol. 24, 396-403.

Hawkins, B.T., Davis, T.P., 2005. The blood-brain barrier/neurovascular unit in health and disease. Pharmacol Rev. 57, 173-185.

Heinemann, U., Lux, H.D., 1975. Undershoots following stimulus-induced rises of extracellular potassium concentration in cerebral cortex of cat. Brain Res. 93, 63-76.

Helmchen, F., Denk, W., 2005. Deep tissue two-photon microscopy. Nat. Methods 2, 932-940.

Hill, J.M., Clement, C., Zhao, Y., Lukiw, W.J., 2015. Induction of the pro-inflammatory NF-kBsensitive miRNA-146a by human neurotrophic viruses. Front. Microbiol. 6, 43.

His, W., 1865. Über ein perivasculäres Canalsystem in den nervosen central-Organen und über dessen Beziehungen aum Lymphsystem. Z. Wiss. Zool. 15, 127-141.

Hladky, S.B., Barrand M.A., 2014. Mechanisms of fluid movement into, through and out of the brain: evaluation of the evidence. Fluids Barriers CNS 11, 26.

Hökfelt, T., Kellerth, J.O., Nilsson, G., Pernow, B., 1975. Substance p: localization in the central nervous system and in some primary sensory neurons. Science 190, 889-890.

Holloway, G.A., Jr., Watkins, D.W., 1977. Laser Doppler measurement of cutaneous blood flow. J. Invest. Dermatol. 69, 306-309. 
Horan, K.L., Adamski, S.W., Ayele, W., Langone, J.J., Grega, G.J., 1986. Evidence that prolonged histamine suffusions produce transient increases in vascular permeability subsequent to the formation of venular macromolecular leakage sites. Proof of the Majno-Palade hypothesis. Am. J. Pathol. 123, 570-576.

Huang, D., Li, S., Dhaka, A., Story, G.M., Cao, Y.Q., 2012. Expression of the transient receptor potential channels TRPV1, TRPA1 and TRPM8 in mouse trigeminal primary afferent neurons innervating the dura. Mol. Pain 8, 66.

Huang, T.Y., Chung, H.W., Chen, M.Y., Giiang, L.H., Chin, S.C., Lee, C.S., Chen, C.Y., Liu, Y.J., 2004. Supratentorial cerebrospinal fluid production rate in healthy adults: quantification with two-dimensional cine phase-contrast MR imaging with high temporal and spatial resolution. Radiology 233, 603-608.

Hughes, T.M., Kuller, L.H., Barinas-Mitchell, E.J., McDade, E.M., Klunk, W.E., Cohen, A.D., Mathis, C.A., Dekosky, S.T., Price, J.C., Lopez, O.L., 2014. Arterial stiffness and betaamyloid progression in nondemented elderly adults. JAMA Neurol. 71, 562-568.

Huber, G.C., 1899. Observations on the innervatlon of the intracranial vessels. J. Comp. Neurol. 9, 1-34.

Hume, D.A., Freeman, T.C., 2014. Transcriptomic analysis of mononuclear phagocyte differentiation and activation. Immunol. Rev. 262, 74-84.

Hume, D.A., Mabbott, N., Raza, S., Freeman, T.C., 2013. Can DCs be distinguished from macrophages by molecular signatures? Nat. Immunol. 14, 187-189.

Husted, R.F., Reed, D.J., 1976. Regulation of cerebrospinal fluid potassium by the cat choroid plexus. J. Physiol. 25, 213-221.

Husted, R.F., Reed, D.J., 1977. Regulation of cerebrospinal fluid bicarbonate by the cat choroid plexus. J. Physiol. 267, 411-428.

Iadecola, C., Nedergaard, M., 2007. Glial regulation of the cerebral microvasculature. Nat. Neurosci. 10, 1369-1376.

Ibanez-Tallon, I., Pagenstecher, A., Fliegauf, M., Olbrich, H., Kispert, A., Ketelsen, U.P., North, A., Heintz, N., Omran, H., 2004. Dysfunction of axonemal dynein heavy chain Mdnah5 inhibits ependymal flow and reveals a novel mechanism for hydrocephalus formation. Hum. Mol. Genet. 13, 2133-2141.

Ichimura, T., Fraser, P.A., Cserr, H.F., 1991. Distribution of extracellular tracers in perivascular spaces of the rat brain. Brain Res. 545, 103-113.

Iliff, J.J., Lee, H., Yu, M., Feng, T., Logan, J., Nedergaard, M., Benveniste, H., 2013a. Brain-wide pathway for waste clearance captured by contrast-enhanced MRI. J. Clin. Invest. 123, 12991309.

Iliff, J.J., Wang, M., Liao, Y., Plogg, B.A., Peng, W., Gundersen, G.A., Benveniste, H., Vates, G.E., Deane, R., Goldman, S.A., Nagelhus, E.A., Nedergaard, M., 2012. A paravascular pathway facilitates CSF flow through the brain parenchyma and the clearance of interstitial solutes, including amyloid beta. Sci. Transl. Med. 4, 147ra111.

Iliff, J.J., Wang, M., Zeppenfeld, D.M., Venkataraman, A., Plog, B.A., Liao, Y., Deane, R., Nedergaard, M., 2013b. Cerebral arterial pulsation drives paravascular CSF-interstitial fluid exchange in the murine brain. J. Neurosci. 33, 18190-18199.

Immig, K., Gericke, M., Menzel, F., Merz, F., Krueger, M., Schiefenhovel, F., Losche, A., Jager, K., Hanisch, U.K., Biber, K., Bechmann, I., 2015. CD11c-positive cells from brain, spleen, lung, and liver exhibit site-specific immune phenotypes and plastically adapt to new environments. Glia 63, 611-625.

Ishii, T., Ishii, M., 2011. Intravital two-photon imaging: a versatile tool for dissecting the immune system. Ann. Rheum. Dis. 70 Suppl 1, i113-115.

Isshiki, M., Okabe, S., 2014. Evaluation of cranial window types for in vivo two-photon imaging of brain microstructures. Microscopy (Oxf) 63, 53-63. 
Iwanow, G., Romodanowsky, K., 1928. Über den anatomischen Zusammenhang der cerebralen und spinalen submeningealen Räume mit dem Lymphsystem. I. Methodik und wichtigste Beobachtungen. Z. ges. exp. Med. 58, 596 - 607.

Jackson, J.L., Cogbill, E., Santana-Davila, R., Eldredge, C., Collier, W., Gradall, A., Sehgal N., Kuester, J., 2015. A Comparative Effectiveness Meta-Analysis of Drugs for the Prophylaxis of Migraine Headache. PLoS One 10, e0130733.

Jacobi, W., 1924. Das Saftspaltensystem der Dura. Arch. Psychiatr. Nervenkr. 70, 269-285.

Jacobi, W., Magnus, G., 1925. Pharmakologische Beeinflussung des Hirnwassers. Arch. Psychiat. $74,126-138$.

Jansen, I., 1992. Characterization of calcitonin gene-related peptide (CGRP) receptors in guinea pig basilar artery. Neuropeptides 21, 73-79.

Jansen, I., Uddman, R., Ekman, R., Olesen, J., Ottosson, A., Edvinsson, L., 1992. Distribution and effects of neuropeptide $\mathrm{Y}$, vasoactive intestinal peptide, substance $\mathrm{P}$, and calcitonin generelated peptide in human middle meningeal arteries: comparison with cerebral and temporal arteries. Peptides 13, 527-536.

Jansen-Olesen, I., Baun, M., Amrutkar, D.V., Ramachandran, R., Christophersen, D.V., Olesen, J., 2014. PACAP-38 but not VIP induces release of CGRP from trigeminal nucleus caudalis via a receptor distinct from the PAC1 receptor. Neuropeptides 48, 53-64.

Janzer, R.C., Raff, M.C., 1987. Astrocytes induce blood-brain barrier properties in endothelial cells. Nature 325, 253-257.

Jefferson, G., Stewart, D., 1928. On the veins of the diploë. Br. J. Surg. 16, 70-88.

Jessen, N.A., Munk, A.S., Lundgaard, I., Nedergaard, M., 2015. The glymphatic system: a beginner's guide. Neurochem. Res. 40, 2583-2599.

Jiang, B., Bezhadian, M.A., Caldwell, R.B., 1995. Astrocytes modulate retinal vasculogenesis: effects on endothelial cell differentiation. Glia 15, 1-10.

Jiang, J., Tyssebotn, I., 1997. Measurement of cerebrospinal fluid pressure in conscious rats. Undersea Hyperb. Med. 24, 39-43.

Johanson, C.E., Duncan, J.A., 3rd, Klinge, P.M., Brinker, T., Stopa, E.G., Silverberg, G.D., 2008. Multiplicity of cerebrospinal fluid functions: New challenges in health and disease. Cerebrospinal Fluid Res. 5, 10.

Johansson, P.A., 2014. The choroid plexuses and their impact on developmental neurogenesis. Front. Neurosci. 8, 340.

Johnson, D., Krenger, W., 1992. Interactions of mast cells with the nervous system--recent advances. Neurochem. Res. 17, 939-951.

Jones. E.G., 1970. On the mode of entry of blood vessels into the cerebral cortex. J. Anat. 106, 507520.

Jones, H.C., Gratton, J.A., 1989. The effect of cerebrospinal fluid pressure on dural venous pressure in young rats. J. Neurosurg. 71, 119-123.

Jones, S.J., Boyde, A., Pawley, J.B., 1975. Osteoblasts and collagen orientation. Cell Tissue Res. 159, 73-80.

Jung, S., Aliberti, J., Graemmel, P., Sunshine, M.J., Kreutzberg, G.W., Sher, A., Littman, D.R., 2000. Analysis of fractalkine receptor CX(3)CR1 function by targeted deletion and green fluorescent protein reporter gene insertion. Mol. Cell Biol. 20, 4106-4114.

Kacem, K., Lacombe, P., Seylaz, J., Bonvento, G., 1998. Structural organization of the perivascular astrocyte endfeet and their relationship with the endothelial glucose transporter: a confocal microscopy study. Glia 23, 1-10.

Kageneck, C., Nixdorf-Bergweiler, B.E., Messlinger, K., Fischer, M.J., 2014. Release of CGRP from mouse brainstem slices indicates central inhibitory effect of triptans and kynurenate. $\mathrm{J}$. Headache Pain 15, 7.

Kai-Kai, M.A., Howe, R., 1991. Glutamate-immunoreactivity in the trigeminal and dorsal root ganglia, and intraspinal neurons and fibres in the dorsal horn of the rat. Histochem. J. 23, 171-179. 
Kang, S.S., McGavern, D.B., 2009. Inflammation on the mind: visualizing immunity in the central nervous system. Curr. Top. Microbiol. Immunol. 334, 227-263.

Kazmi, S.M., Richards, L.M., Schrandt, C.J., Davis, M.A., Dunn, A.K., 2015. Expanding applications, accuracy, and interpretation of laser speckle contrast imaging of cerebral blood flow. J. Cereb. Blood Flow Metab. 35, 1076-1084.

Keaney, J., Campbell, M., 2015. The dynamic blood-brain barrier. FEBS J. 282, 4067-4079.

Keep, R.F., Jones, H.C., 1990. A morphometric study on the development of the lateral ventricle choroid plexus, choroid plexus capillaries and ventricular ependyma in the rat. Brain Res. Dev. Brain. Res. 56, 47-53.

Keller, J.T., Dimlich, R.V., Zuccarello, M., Lanker, L., Strauss, T.A., Fritts, M.J., 1991. Influence of the sympathetic nervous system as well as trigeminal sensory fibres on rat dural mast cells. Cephalalgia 11, 215-221.

Keller, J.T., Marfurt, C.F., 1991. Peptidergic and serotoninergic innervation of the rat dura mater. J. Comp. Neurol. 309, 515-534.

Keller, J.T., Marfurt, C.F., Dimlich, R.V., Tierney, B.E., 1989. Sympathetic innervation of the supratentorial dura mater of the rat. J. Comp. Neurol. 290, 310-321.

Kerber, C.W., Newton, T.H., 1973. The macro and microvasculature of the dura mater. Neuroradiology 6, 175-179.

Kettenmann, H., Hanisch, U.K., Noda, M., Verkhratsky, A., 2011. Physiology of microglia. Physiol. Rev. 91, 461-553.

Key, A., Retzius, G. (1875) Studien in der Anatomie des Nervensystems und des Bindegewebes. Stockholm: Samson \& Waller.

Kida, S., Pantazis, A., Weller, R.O., 1993a. CSF drains directly from the subarachnoid space into nasal lymphatics in the rat. Anatomy, histology and immunological significance. Neuropath. App. Neurobiol. 19, 480-488.

Kida, S., Steart P.V., Zhang, E.T., Weller, R.O., 1993b. Perivascular cells act as scavengers in the cerebral perivascular spaces and remain distinct from pericytes, microglia and macrophages. Acta Neuropathol. 8, 646-652.

Kim, D.S., Choi, J.U., Huh, R., Yun, P.H., Kim, D.I., 1999. Quantitative assessment of cerebrospinal fluid hydrodynamics using a phase-contrast cine MR image in hydrocephalus. Childs Nerv. Syst. 15, 461-467.

Kim, J.V., Dustin, M.L., 2006. Innate response to focal necrotic injury inside the blood-brain barrier. J. Immunol. 177, 5269-5277.

Kim, J.V., Kang, S.S., Dustin, M.L., McGavern, D.B., 2009. Myelomonocytic cell recruitment causes fatal CNS vascular injury during acute viral meningitis. Nature 457, 191-195.

Kivisakk, P., Imitola, J., Rasmussen, S., Elyaman, W., Zhu, B., Ransohoff, R.M., Khoury, S.J., 2009. Localizing central nervous system immune surveillance: meningeal antigenpresenting cells activate T cells during experimental autoimmune encephalomyelitis. Ann. Neurol. 65, 457-469.

Kivisakk, P., Mahad, D.J., Callahan, M.K., Trebst, C., Tucky, B., Wei, T., Wu, L., Baekkevold, E.S., Lassmann, H., Staugaitis, S.M., Campbell, J.J., Ransohoff, R.M., 2003. Human cerebrospinal fluid central memory CD4+ T cells: evidence for trafficking through choroid plexus and meninges via P-selectin. Proc. Natl Acad. Sci. U S A 100, 8389-8394.

Klatzo, I., Miquel, J., Ferris, P.J., Prokop, J.D., Smith, D.E., 1964. Observations on the passage of the fluorescein labeled serum proteins (Flsp) from the cerebrospinal fluid. J. Neuropathol. Exp. Neurol. 23, 18-35.

Kleinfeld D., Mitra P.P., Helmchen F., Denk W., 1998. Fluctuations and stimulus-induced changes in blood flow observed in individual capillaries in layers 2 through 4 of rat neocortex. Proc. Natl Acad. Sci. U S A 95, 15741-15746.

Klohs, J., Rudin, M., Shimshek, D.R., Beckmann, N., 2014. Imaging of cerebrovascular pathology in animal models of Alzheimer's disease. Front. Aging Neurosci. 6, 32. 
Knobloch, V., Binter, C., Kurtcuoglu, V., Kozerke, S., 2014. Arterial, venous, and cerebrospinal fluid flow: simultaneous assessment with Bayesian multipoint velocity-encoded MR imaging. Radiology 270, 566-573.

Knotkova, H., Pappagallo, M., 2007. Imaging intracranial plasma extravasation in a migraine patient: a case report. Pain Med. 8, 383-387.

Kobayashi, H., Minami, S., Itoh, S., Shiraishi, S., Yokoo, H., Yanagita, T., Uezono, Y., Mohri, M., Wada, A., 2001. Aquaporin subtypes in rat cerebral microvessels. Neurosci. Lett. 297, $163-$ 166.

Kobayashi, S., Kyoshima, K., Olschowka, J.A., Jacobowitz, D.M., 1983. Vasoactive intestinal polypeptide immunoreactive and cholinergic nerves in the whole mount preparation of the major cerebral arteries of the rat. Histochemistry 79, 377-381.

Kobayashi, S., Waltz, A.G., Rhoton, A.L., Jr., 1971. Effects of stimulation of cervical sympathetic nerves on cortical blood flow and vascular reactivity. Neurology 21, 297-302.

Koh, L., Zakharov, A., Johnston, M., 2005. Integration of the subarachnoid space and lymphatics: is it time to embrace a new concept of cerebrospinal fluid absorption? Cerebrospinal Fluid Res. 2, 6.

Kölliker, A. (1867) Hüllen und Gefässe des zentralen Nervensystems. In: Handbuch der Gewebelehre des Menschen, pp 307-316 Leipzig: Wilhelm Engelmann.

Kosaras, B., Jakubowski, M., Kainz, V., Burstein, R., 2009. Sensory innervation of the calvarial bones of the mouse. J. Comp. Neurol. 515, 331-348.

Kousi, M., Katsanis, N., 2016. The genetic basis of hydrocephalus. Annu. Rev. Neurosci. 39, 409435.

Krahn, V., 1982. The pia mater at the site of entry of blood vessels into the central nervous system. Anat. Embryol. 164, 257-263.

Kress, B.T., Iliff, J.J., Xia, M., Wang, M., Wei, H.S., Zeppenfeld, D., Xie, L., Kang, H., Xu, Q., Liew, J.A., Plog, B.A., Ding, F., Deane, R., Nedergaard, M., 2014. Impairment of paravascular clearance pathways in the aging brain. Ann. Neurol. 76, 845-861.

Kreutzberg, G.W., 1996. Microglia: a sensor for pathological events in the CNS. Trends Neurosci. 19, 312-318.

Krisch, B. 1988. Ultrastructure of the meninges at the site of penetration of veins through the dura mater, with particular reference to Pacchionian granulations. Investigations in the rat and two species of New-World monkeys (Cebus apella, Callitrix jacchus). Cell Tiss. Res. 251, 621-631.

Krisch, B., Leonhardt, H., Oksche, A., 1983. The meningeal compartments of the median eminence and the cortex. A comparative analysis in the rat. Cell Tissue Res. 228, 597-640.

Krisch, B., Leonhardt, H., Oksche, A., 1984. Compartments and perivascular arrangement of the meninges covering the cerebral cortex of the rat. Cell Tissue Res. 238, 459-474.

Krstić, R.V., 1988, Die Gewebe des Menschen und der Säugetiere: Springer.

Kubo, Y., Ohtsuki, S., Uchida, Y., Terasaki, T., 2015. Quantitative determination of luminal and abluminal membrane distributions of transporters in porcine brain capillaries by plasma membrane fractionation and quantitative targeted proteomics. J. Pharm. Sci. 104, 30603068.

Kuerten, S., Javeri, S., Tary-Lehmann, M., Lehmann, P.V., Angelov, D.N., 2008. Fundamental differences in the dynamics of CNS lesion development and composition in MP4- and MOG peptide 35-55-induced experimental autoimmune encephalomyelitis. Clin. Immunol. 129, 256-267.

Kuerten, S., Schickel, A., Kerkloh, C., Recks, M.S., Addicks, K., Ruddle, N.H., Lehmann, P.V., 2012. Tertiary lymphoid organ development coincides with determinant spreading of the myelin-specific T cell response. Acta Neuropathol. 124, 861-873.

Kume, T., Deng, K.Y., Winfrey, V., Gould, D.B., Walter, M.A., Hogan, B.L., 1998. The forkhead/winged helix gene Mf1 is disrupted in the pleiotropic mouse mutation congenital hydrocephalus. Cell 93, 985-996. 
Kurosawa, M., Messlinger, K., Pawlak, M., Schmidt, R.F., 1995. Increase of meningeal blood flow after electrical stimulation of rat dura mater encephali: mediation by calcitonin gene-related peptide. Br. J. Pharmacol. 114, 1397-1402.

Laman, J.D., Weller, R.O., 2013. Drainage of cells and soluble antigen from the CNS to regional lymph nodes. J. Neuroimmune Pharmacol. 8, 840-856.

Lamers, S.L., Gray, R.R., Salemi, M., Huysentruyt, L.C., McGrath, M.S., 2011. HIV-1 phylogenetic analysis shows HIV-1 transits through the meninges to brain and peripheral tissues. Infect. Genet. Evol. 11, 31-37.

Landis, D.M., Reese, T.S., 1981. Membrane structure in mammalian astrocytes: a review of freezefracture studies on adult, developing, reactive and cultured astrocytes. J. Exp. Biol. 95, 3548.

Lassen, N.A., 1968. Brain extracellular $\mathrm{pH}$ : the main factor controlling cerebral blood flow. Scand. J. Clin. Lab. Invest. 22, 247-251.

Lauterbach, H., Zuniga, E.I., Truong, P., Oldstone, M.B., McGavern, D.B., 2006. Adoptive immunotherapy induces CNS dendritic cell recruitment and antigen presentation during clearance of a persistent viral infection. J. Exp. Med. 203, 1963-1975.

Leao, A.A.P., 1944a. Pial circulation and spreading depression of activity in the cerebral cortex. J. Neurophysiol. 7, 391-396.

Leao, A.A.P., 1944b. Spreading depression of activity in the cerebral cortex. J. Neurophysiol. 7, 359-390.

Leao, A.A.P., Morrison, R.S., 1945. Propagation of spreading cortical depression. J Neurophysiol. 8, 33-45.

Leblanc, G.G., Trimmer, B.A., Landis, S.C., 1987. Neuropeptide Y-like immunoreactivity in rat cranial parasympathetic neurons: coexistence with vasoactive intestinal peptide and choline acetyltransferase. Proc. Natl Acad. Sci. U S A 84, 3511-3515.

Lee, Y., Kawai, Y., Shiosaka, S., Takami, K., Kiyama, H., Hillyard, C.J., Girgis, S., MacIntyre, I., Emson, P.C., Tohyama, M., 1985a. Coexistence of calcitonin gene-related peptide and substance P-like peptide in single cells of the trigeminal ganglion of the rat: immunohistochemical analysis. Brain Res. 330, 194-196.

Lee, Y., Takami, K., Kawai, Y., Girgis, S., Hillyard, C.J., MacIntyre, I., Emson, P.C., Tohyama, M., 1985b. Distribution of calcitonin gene-related peptide in the rat peripheral nervous system with reference to its coexistence with substance P. Neuroscience 15, 12271237.

Lehre, K.P., Levy, L.M., Ottersen, O.P., Storm-Mathisen, J., Danbolt, N.C., 1995. Differential expression of two glial glutamate transporters in the rat brain: quantitative and immunocytochemical observations. J. Neurosci. 15, 1835-1853.

Lennerz, J.K., Ruhle, V., Ceppa, E.P., Neuhuber, W.L., Bunnett, N.W., Grady, E.F., Messlinger, K., 2008. Calcitonin receptor-like receptor (CLR), receptor activity-modifying protein 1 (RAMP1), and calcitonin gene-related peptide (CGRP) immunoreactivity in the rat trigeminovascular system: differences between peripheral and central CGRP receptor distribution. J. Comp. Neurol. 507, 1277-1299.

Levy, D., Burstein, R., Kainz, V., Jakubowski, M., Strassman, A.M., 2007. Mast cell degranulation activates a pain pathway underlying migraine headache. Pain 130, 166-176.

Lewandowsky, M., 1900. Zur Lehre von der Cerebrospinalflüssigkeit. Zeitschr. klin. Med. 40, 480 $494+485$ plates.

Li, M.D., Kane, J.K., Matta, S.G., Blaner, W.S., Sharp, B.M., 2000. Nicotine enhances the biosynthesis and secretion of transthyretin from the choroid plexus in rats: implications for beta-amyloid formation. J. Neurosci. 20, 1318-1323.

Li, Y., Baran, U., Wang, R.K., 2014. Application of thinned-skull cranial window to mouse cerebral blood flow imaging using optical microangiography. PLoS One 9, e113658.

Liang, R., Broussard, G.J., Tian, L., 2015. Imaging chemical neurotransmission with genetically encoded fluorescent sensors. ACS Chem. Neurosci. 6, 84-93. 
Lim, A.T., Chandra, R.V., Trost, N.M., McKelvie, P.A., Stuckey, S.L., 2015. Large anterior temporal Virchow-Robin spaces: unique MR imaging features. Neuroradiology 57, 491-499.

Lindquist, R.L., Shakhar, G., Dudziak, D., Wardemann, H., Eisenreich, T., Dustin, M.L., Nussenzweig, M.C., 2004. Visualizing dendritic cell networks in vivo. Nat. Immunol. 5, 1243-1250.

Liu-Chen, L.Y., Han, D.H., Moskowitz, M.A., 1983. Pia arachnoid contains substance P originating from trigeminal neurons. Neuroscience 9, 803-808.

Lochhead, J.J., Wolak, D.J., Pizzo, M.E., Thorne, R.G., 2015. Rapid transport within cerebral perivascular spaces underlies widespread tracer distribution in the brain after intranasal administration. J. Cereb. Blood Flow Metab. 35, 371-381.

Locke, C.E., Naffziger, H.C., 1924. The cerebral subarachnoid system. Arch. Neurol. Psychiat.12, 411-418.

Lorenzo, J., Horowitz, M., Choi, Y., 2008. Osteoimmunology: interactions of the bone and immune system. Endocr. Rev. 29, 403-440.

Loscher, W., Potschka, H., 2005. Role of drug efflux transporters in the brain for drug disposition and treatment of brain diseases. Prog. Neurobiol. 76, 22-76.

Louveau, A., Smirnov, I., Keyes, T.J., Eccles, J.D., Rouhani, S.J., Peske, J.D., Derecki, N.C., Castle, D., Mandell, J.W., Lee, K.S., Harris, T.H., Kipnis, J., 2015. Structural and functional features of central nervous system lymphatic vessels. Nature 523, 337-341.

Lowry, J.P., O'Neill, R.D., Boutelle, M.G., Fillenz, M., 1998. Continuous monitoring of extracellular glucose concentrations in the striatum of freely moving rats with an implanted glucose biosensor. J. Neurochem. 70, 391-396.

Luetmer, P.H., Huston, J., Friedman, J.A., Dixon, G.R., Petersen, R.C., Jack, C.R., McClelland, R.L., Ebersold, M.J., 2002. Measurement of cerebrospinal fluid flow at the cerebral aqueduct by use of phase-contrast magnetic resonance imaging: technique validation and utility in diagnosing idiopathic normal pressure hydrocephalus. Neurosurgery 50, 534-543; discussion 543-534.

Luschka, H. (1850) Die Nerven der Harten Hirnhaut. Tübingen: H. Laupp.

Ma, Q.P., Hill, R., Sirinathsinghji, D., 2001. Colocalization of CGRP with 5-HT1B/1D receptors and substance $\mathrm{P}$ in trigeminal ganglion neurons in rats. Eur. J. Neurosci. 13, 2099-2104.

Magendie F., 1825. Mémoire sur un liquide qui se trouve dans le crane et le canal vertébrale de l'homme et des animaux mammifères. Journal de Physiologie expérimentale et pathologique $5,27-37$.

Magendie F. (1842) Recherches physiologiques et cliniques sur le liquide céphalo-rachidien. Paris: Librarie médicale de Méquignon-Marvis.

Maher, B.A., Ahmed, I.A., Karloukovski, V., MacLaren, D.A., Foulds, P.G., Allsop, D., Mann, D.M., Torres-Jardon, R., Calderon-Garciduenas, L., 2016. Magnetite pollution nanoparticles in the human brain. Proc. Natl Acad. Sci. U S A, 113, 10797-10801.

Majno, G., Karnovsky, M.L., 1961. A biochemical and morphologic study of myelination and demyelination. III. Effect of an organo-phosphorus compound (Mipafox) on the biosynthesis of lipid by nervous tissue of rats and hens. J. Neurochem. 8, 1-16.

Majno, G., Palade, G.E., Schoefl, G.I., 1961. Studies on inflammation. II. The site of action of histamine and serotonin along the vascular tree: a topographic study. J. Biophys. Biochem. Cytol. 11, 607-626.

Mandell, E.C., Zimmermann, E., 1980. Continuous measurement of cerebrospinal fluid pressure in unrestrained rats. Physiol. Behav. 24, 399-402.

Mann, J.D., Butler, A.B., Rosenthal, J.E., Maffeo, C.J., Johnson, R.N., Bass, N.H., 1978. Regulation of intracranial pressure in rat, dog, and man. Ann. Neurol. 3, 156-165.

Marker, D.F., Tremblay, M.E., Lu, S.M., Majewska, A.K., Gelbard, H.A., 2010. A thin-skull window technique for chronic two-photon in vivo imaging of murine microglia in models of neuroinflammation. J. Vis. Exp. 43, pii:2059. 
Markowitz, S., Saito, K., Buzzi, M.G., Moskowitz, M.A., 1989. The development of neurogenic plasma extravasation in the rat dura mater does not depend upon the degranulation of mast cells. Brain Res. 477, 157-165.

Markowitz, S., Saito, K., Moskowitz, M.A., 1987. Neurogenically mediated leakage of plasma protein occurs from blood vessels in dura mater but not brain. J. Neurosci. 7, 4129-4136.

Marom, Z., Casale, T.B., 1983. Mast cells and their mediators. Ann. Allergy 50, 367-370.

Mascagni, P. (1787) Vasorum lymphaticorum corporis humani historia et ichnographia. Siena: Pazzini Carli.

Masuda, H., Ushiyama, A., Hirota, S., Lawlor, G.F., Ohkubo, C., 2007. Long-term observation of pial microcirculatory parameters using an implanted cranial window method in the rat. In Vivo 21, 471-479.

Mathiisen, T.M., Lehre, K.P., Danbolt, N.C., Ottersen, O.P., 2010. The perivascular astroglial sheath provides a complete covering of the brain microvessels: an electron microscopic 3D reconstruction. Glia 58, 1094-1103.

Mathis, A.M., Bridges, A.S., Ismail, M.A., Kumar, A., Francesconi, I., Anbazhagan, M., Hu, Q., Tanious, F.A., Wenzler, T., Saulter, J., Wilson, W.D., Brun, R., Boykin, D.W., Tidwell, R.R., Hall, J.E., 2007. Diphenyl furans and aza analogs: effects of structural modification on in vitro activity, DNA binding, and accumulation and distribution in trypanosomes. Antimicrob. Agents Chemother. 51, 2801-2810.

Mathis, A.M., Holman, J.L., Sturk, L.M., Ismail, M.A., Boykin, D.W., Tidwell, R.R., Hall, J.E., 2006. Accumulation and intracellular distribution of antitrypanosomal diamidine compounds DB75 and DB820 in African trypanosomes. Antimicrob. Agents Chemother. 50, 2185-2191.

Mathur, A., Khanna, N., Chaturvedi, U.C., 1992. Breakdown of blood-brain barrier by virusinduced cytokine during Japanese encephalitis virus infection. Int. J. Exp. Pathol. 73, 603611.

Mato, M., Ookawara, S., Sakamoto, A., Aikawa, E., Ogawa, T., Mitsuhashi, U., Masuzawa, T., Suzuki, H., Honda, M., Yazaki, Y., Watanabe, E., Luoma, J., Yla-Herttuala, S., Fraser, I., Gordon, S., Kodama, T., 1996. Involvement of specific macrophage-lineage cells surrounding arterioles in barrier and scavenger function in brain cortex. Proc. Natl Acad. Sci. U S A 93, 3269-3274.

Matsuyama, T., Shiosaka, S., Matsumoto, M., Yoneda, S., Kimura, K., Abe, H., Hayakawa, T., Inoue, H., Tohyama, M., 1983. Overall distribution of vasoactive intestinal polypeptidecontaining nerves on the wall of cerebral arteries: an immunohistochemical study using whole-mounts. Neuroscience 10, 89-96.

Mayberg, M.R., Zervas, N.T., Moskowitz, M.A., 1984. Trigeminal projections to supratentorial pial and dural blood vessels in cats demonstrated by horseradish peroxidase histochemistry. J. Comp. Neurol. 223, 46-56.

Maynard, E.A., Schultz, R.L., Pease, D.C., 1957. Electron microscopy of the vascular bed of rat cerebral cortex. Am. J. Anat. 100, 409-433.

McCaffrey, T.V., McCook, R.D., Wurster, R.D., 1975. Effect of head skin temperature on tympanic and oral temperature in man. J. Appl. Physiol. 39, 114-118.

McColl, S.R., Staykova, M.A., Wozniak, A., Fordham, S., Bruce, J., Willenborg, D.O., 1998. Treatment with anti-granulocyte antibodies inhibits the effector phase of experimental autoimmune encephalomyelitis. J. Immunol. 161, 6421-6426.

McKittrick, C.M., Lawrence, C.E., Carswell, H.V., 2015. Mast cells promote blood brain barrier breakdown and neutrophil infiltration in a mouse model of focal cerebral ischemia. J. Cereb. Blood Flow Metab. 35, 638-647.

McMenamin, P.G., 1999. Distribution and phenotype of dendritic cells and resident tissue macrophages in the dura mater, leptomeninges, and choroid plexus of the rat brain as demonstrated in wholemount preparations. J. Comp. Neurol. 405, 553-562. 
McMenamin, P.G., Wealthall, R.J., Deverall, M., Cooper, S.J., Griffin, B., 2003. Macrophages and dendritic cells in the rat meninges and choroid plexus: three-dimensional localisation by environmental scanning electron microscopy and confocal microscopy. Cell Tissue Res. 313, 259-269.

Medawar, P.B., 1948. Immunity to homologous grafted skin; the fate of skin homografts transplanted to the brain, to subcutaneous tissue, and to the anterior chamber of the eye. $\mathrm{Br}$. J. Exp. Pathol. 29, 58-69.

Mekori, Y.A., Metcalfe, D.D., 1999. Mast cell-T cell interactions. J. Allergy Clin. Immunol. 104, 517-523.

Menasria, R., Canivet,C., Piret, J., Boivin, G., 2015. Infiltration Pattern of Blood Monocytes into the Central Nervous System during Experimental Herpes Simplex Virus Encephalitis. PLoS One 10, e0145773.

Merad, M., Sathe, P., Helft, J., Miller, J., Mortha, A., 2013. The dendritic cell lineage: ontogeny and function of dendritic cells and their subsets in the steady state and the inflamed setting. Annu. Rev. Immunol. 31, 563-604.

Mercier, F., Hatton, G.I., 2000. Immunocytochemical basis for a meningeo-glial network. J. Comp. Neurol. 420, 445-465.

Meredith, M.M., Liu, K., Darrasse-Jeze, G., Kamphorst, A.O., Schreiber, H.A., Guermonprez, P., Idoyaga, J., Cheong, C., Yao, K.H., Niec, R.E., Nussenzweig, M.C., 2012. Expression of the zinc finger transcription factor zDC (Zbtb46, Btbd4) defines the classical dendritic cell lineage. J. Exp. Med. 209, 1153-1165.

Messlinger, K., 2009. Migraine: where and how does the pain originate? Exp. Brain Res. 196,179193.

Messlinger, K., Hanesch, U., Baumgartel, M., Trost, B., Schmidt, R.F., 1993. Innervation of the dura mater encephali of cat and rat: ultrastructure and calcitonin gene-related peptide-like and substance P-like immunoreactivity. Anat. Embryol. (Berl.) 188, 219-237.

Messlinger, K, Suzuki, A, Pawlak, M, Zehnter, A, Schmidt, RF., 2000. Involvement of nitric oxide in the modulation of dural arterial blood flow in the rat. Br. J. Pharmacol. 129, 1397404.

Milhorat, T.H., 1975. The third circulation revisited. J. Neurosurg. 42, 628-645.

Milhorat, T.H., Hammock, M.K., Fenstermacher, J.D., Levin, V.A., 1971. Cerebrospinal fluid production by the choroid plexus and brain. Science 173, 330-332.

Miller, F.N., Sims, D.E., 1986. Contractile elements in the regulation of macromolecular permeability. Fed. Proc. 45, 84-88.

Moghaddam, B., Adams, R.N., 1987. Regional differences in resting extracellular potassium levels of rat brain. Brain Res. 406, 337-340.

Mohler,W., Millard, A.C., Campagnola, P.J., 2003. Second harmonic generation imaging of endogenous structural proteins. Methods 29, 97-109.

Mokgokong, R., Wang, S., Taylor, C.J., Barrand, M.A., Hladky, S.B., 2014. Ion transporters in brain endothelial cells that contribute to formation of brain interstitial fluid. Pflugers Arch. 466, 887-901.

Möller, K., Zhang, Y.Z., Hakanson, R., Luts, A., Sjolund, B., Uddman, R., Sundler, F., 1993. Pituitary adenylate cyclase activating peptide is a sensory neuropeptide: immunocytochemical and immunochemical evidence. Neuroscience 57, 725-732.

Morishima, T., Hayashi, K., 1978. Meningeal exudate cells in vaccinia meningitis of mice: role of local T cells. Infect. Immun. 20, 752-759.

Morse, D.E., Low, F.N., 1972. The fine structure of the pia mater of the rat. Am. J. Anat. 133, 349367.

Mortazavi, M.M., Denning, M., Yalcin, B., Shoja, M.M., Loukas, M., Tubbs, R.S., 2013. The intracranial bridging veins: a comprehensive review of their history, anatomy, histology, pathology, and neurosurgical implications. Childs Nerv. Syst. 29, 1073-1078. 
Mortazavi, M.M., Tubbs, R.S., Riech, S., Verma, K., Shoja, M.M., Zurada, A., Benninger, B., Loukas, M., Cohen Gadol, A.A., 2012. Anatomy and pathology of the cranial emissary veins: a review with surgical implications. Neurosurgery 70, 1312-1318; discussion 13181319.

Motavkin, P.A., Chertok, V.M., Shul'ga, S.D., 1979. [Effect of acetylcholine on the mast cells of the dura mater]. Biull. Eksp. Biol. Med. 87, 489-491.

Motoike, T., Loughna, S., Perens, E., Roman, B.L., Liao, W., Chau, T.C., Richardson, C.D., Kawate, T., Kuno, J., Weinstein, B.M., Stainier, D.Y., Sato, T.N., 2000. Universal GFP reporter for the study of vascular development. Genesis $28,75-81$.

Mott, F.W. (1906) Histological observations on sleeping sickness and other trypanosome infections. In: Reports of the Sleeping Sickness Commission of the Royal Society, vol. VII, p 45 pp. + plates.

Mott, F.W., 1910. The Oliver-Sharpey lectures on the cerebrospinal fluid. Lancet. 176, 1-8.

Myburgh, E., Coles, J.A., Ritchie, R., Kennedy, P.G., McLatchie, A.P., Rodgers, J., Taylor, M.C., Barrett, M.P., Brewer, J.M., Mottram, J.C., 2013. In vivo imaging of trypanosome-brain interactions and development of a rapid screening test for drugs against CNS stage trypanosomiasis. PLoS Negl. Trop. Dis. 7, e2384.

Nabeshima, S., Reese, T.S., Landis, D.M., Brightman, M.W., 1975. Junctions in the meninges and marginal glia. J. Comp. Neurol. 164, 127-169.

Nacer, A., Movila, A., Sohet, F., Girgis, N.M., Gundra, U.M., Loke, P., Daneman, R., Frevert, U., 2014. Experimental cerebral malaria pathogenesis--hemodynamics at the blood brain barrier. PLoS Pathog 10, e1004528.

Nagelhus, E.A., Mathiisen, T.M., Ottersen, O.P., 2004. Aquaporin-4 in the central nervous system: cellular and subcellular distribution and coexpression with KIR4.1. Neuroscience 129, 905913.

Nakagomi, T., Nakano-Doi, A., Matsuyama, T., 2015. Leptomeninges: a novel stem cell niche harboring ischemia-induced neural progenitors. Histol. Histopathol. 30, 391-399.

Nelson, E., Blinzinger, K., Hager, H., 1961. Electron microscopic observations on subarachnoid and perivascular spaces of the Syrian hamster brain. Neurology 11(4)Pt 1, 285-295.

Nelson, E., Rennels, M., 1970. Innervation of intracranial arteries. Brain 93, 475-490.

Nelson, M.T., Cheng, H., Rubart, M., Santana, L.F., Bonev, A.D., Knot, H.J., Lederer, W.J., 1995. Relaxation of arterial smooth muscle by calcium sparks. Science 270, 633-637.

Neumann, J., 1890. Ueber das Vorkommen der sogenannten "Mastzellen" bei pathologischen Veränderungen des Gehirns. Archiv für Pathologie Anatomie und Physiologie Virchows $122,378-380$.

Nicholson, C., Chen, K.C., Hrabetova, S., Tao L., 2000. Diffusion of molecules in brain extracellular space: theory and experiment. Prog. Brain Res. 125, 129-154.

Nicholson, C., Phillips, J.M., 1981. Ion diffusion modified by tortuosity and volume fraction in the extracellular microenvironment of the rat cerebellum. J. Physiol. 321, 225-257.

Nicholson, C., Phillips, J.M., Gardner-Medwin, A.R., 1979. Diffusion from an iontophoretic point source in the brain: role of tortuosity and volume fraction. Brain Res. 169, 580-584.

Nielsen, K.C., Owman, C., 1967. Adrenergic innervation of pial arteries related to the circle of Willis in the cat. Brain Res. 6, 773-776.

Nielsen, S., Nagelhus, E.A., Amiry-Moghaddam, M., Bourque, C., Agre, P., Ottersen, O.P., 1997. Specialized membrane domains for water transport in glial cells: high-resolution immunogold cytochemistry of aquaporin-4 in rat brain. J. Neurosci. 17, 171-180.

Nishimura, D.G., Macovski, A., Pauly, J.M., 1986. Magnetic resonance angiography. IEEE Trans. Med. Imaging. 5, 140-151.

Nitz, W.R., Bradley, W.G., Jr., Watanabe, A.S., Lee, R.R., Burgoyne, B., O'Sullivan, R.M., Herbst, M.D., 1992. Flow dynamics of cerebrospinal fluid: assessment with phase-contrast velocity MR imaging performed with retrospective cardiac gating. Radiology 183, 395-405. 
Noseda, R., Constandil, L., Bourgeais, L., Chalus, M., Villanueva, L., 2010. Changes of meningeal excitability mediated by corticotrigeminal networks: a link for the endogenous modulation of migraine pain. J. Neurosci. 30, 14420-14429.

O'Connor, T.P., van der Kooy, D., 1986. Pattern of intracranial and extracranial projections of trigeminal ganglion cells. J. Neurosci. 6, 2200-2207.

O'Connor, T.P., van der Kooy, D., 1988. Enrichment of a vasoactive neuropeptide (calcitonin gene related peptide) in the trigeminal sensory projection to the intracranial arteries. J. Neurosci. 8, 2468-2476.

Oda, Y., Nakanishi, I., 1984. Ultrastructure of the mouse leptomeninx. J. Comp. Neurol. 225, 448457.

Oda, Y., Nakanishi, I., 1987. Ultrastructure of the caudal portion of the fourth ventricular roof in the mouse. J. Comp. Neurol. 256, 299-307.

Odoardi, F., Sie, C., Streyl, K., Ulaganathan, V.K., Schlager, C., Lodygin, D., Heckelsmiller, K., Nietfeld, W., Ellwart, J., Klinkert, W.E., Lottaz, C., Nosov, M., Brinkmann, V., Spang, R., Lehrach, H., Vingron, M., Wekerle, H., Flugel-Koch, C., Flugel, A., 2012. T cells become licensed in the lung to enter the central nervous system. Nature 488, 675-679.

Ohata, K., Marmarou, A., 1992. Clearance of brain edema and macromolecules through the cortical extracellular space. J. Neurosurg. 77, 387-396.

Oldendorf, W.H., 1971. Brain uptake of radiolabeled amino acids, amines, and hexoses after arterial injection. Am. J. Physiol. 221, 1629-1639.

Olesen, S.P., 1987. Leakiness of rat brain microvessels to fluorescent probes following craniotomy. Acta Physiol. Scand. 130, 63-68.

Oreskovic, D., Klarica, M., 2010. The formation of cerebrospinal fluid: nearly a hundred years of interpretations and misinterpretations. Brain Res. Rev. 64, 241-262.

Oreskovic, D., Klarica, M., 2011. Development of hydrocephalus and classical hypothesis of cerebrospinal fluid hydrodynamics: facts and illusions. Prog. Neurobiol. 94, 238-258.

Oshio, K., Watanabe, H., Song, Y., Verkman, A.S., Manley, G.T., 2005. Reduced cerebrospinal fluid production and intracranial pressure in mice lacking choroid plexus water channel Aquaporin-1. FASEB J. 19, 76-78.

Ottosson, A., Edvinsson, L., 1997. Release of histamine from dural mast cells by substance P and calcitonin gene-related peptide. Cephalalgia 17, 166-174.

Ozen, I., Deierborg, T., Miharada, K., Padel, T., Englund, E., Genove, G., Paul, G., 2014. Brain pericytes acquire a microglial phenotype after stroke. Acta Neuropathol. 128, 381-396.

Page, A.R., Good, R.A., 1958. A clinical and experimental study of the function of neutrophils in the inflammatory response. Am. J. Pathol. 34, 645-669.

Pai, S., Danne, K.J., Qin, J., Cavanagh, L.L., Smith, A., Hickey, M.J., Weninger, W., 2012. Visualizing leukocyte trafficking in the living brain with 2-photon intravital microscopy. Front. Cell Neurosci. 6, 67.

Pardridge, W.M., Boado, R.J., Farrell, C.R., 1990. Brain-type glucose transporter (GLUT-1) is selectively localized to the blood-brain barrier. Studies with quantitative western blotting and in situ hybridization. J. Biol. Chem. 265, 18035-18040.

Pease, D.C., Schultz, R.L., 1958. Electron microscopy of rat cranial meninges. Am. J. Anat. 102, 301-321.

Pedersen. S.H., Ramachandran, R., Amrutkar, D.V., Petersen, S., Olesen, J., Jansen-Olesen, I., 2015. Mechanisms of glyceryl trinitrate provoked mast cell degranulation. Cephalalgia 35, 1287-1297.

Penfield, W., 1935. A contribution to the mechanism of intracranial pain. Proc Assoc. Res. Nervous Mental Dis. 15, 399-416.

Penfield, W., McNaughton, F., 1940. Dural headache and innervation of the dura mater. Arch. Neurol. 44, 43-75.

Penfield, W.G., 1923. Subdural effusion and internal hydrocephalus. Am. J. Dis. Child 26, 383-390. 
Penn, R.D., Basati, S., Sweetman, B., Guo, X., Linninger, A., 2011. Ventricle wall movements and cerebrospinal fluid flow in hydrocephalus. J. Neurosurg. 115, 159-164.

Peppiatt, C.M., Howarth, C., Mobbs, P., Attwell, D., 2006. Bidirectional control of CNS capillary diameter by pericytes. Nature 443, 700-704.

Perez-de-Puig, I., Miro-Mur, F., Ferrer-Ferrer, M., Gelpi, E., Pedragosa, J., Justicia, C., Urra, X., Chamorro, A., Planas, A.M., 2015. Neutrophil recruitment to the brain in mouse and human ischemic stroke. Acta Neuropathol. 129, 239-257.

Perie, L., Naik, S.H., 2015. Toward defining a 'lineage' -The case for dendritic cells. Semin. Cell Dev. Biol. 41, 3-8.

Peroutka, S.J., 2004. Migraine: a chronic sympathetic nervous system disorder. Headache 44, $53-$ 64.

Pesic, M., Bartholomaus, I., Kyratsous, N.I., Heissmeyer, V., Wekerle, H., Kawakami, N., 2013. 2photon imaging of phagocyte-mediated T cell activation in the CNS. J. Clin. Invest. 123, 1192-1201.

Pestalozzi, R. (1849) Über Aneurysmata spuria der kleinen Gehirnarterien und ihren Zuzammenhang mit Apoplexie. Wurtzburg: F. E. Thein. Wurtzburg: F.E. Thein.

Petersen, K.A., Birk, S., Doods, H., Edvinsson, L., Olesen, J., 2004. Inhibitory effect of BIBN4096BS on cephalic vasodilatation induced by CGRP or transcranial electrical stimulation in the rat. Br. J. Pharmacol. 143, 697-704.

Petersen, K.A., Nilsson, E., Olesen, J., Edvinsson, L., 2005. Presence and function of the calcitonin gene-related peptide receptor on rat pial arteries investigated in vitro and in vivo. Cephalalgia 25, 424-432.

Pettit, A., Girard, J., 1901. Processus sécretoires dans les cellules de revêtement des plexus choroïdes des ventricules latéraux, consecutifs à l'administration de muscarine et d'ether. CR Soc. Biol. 53, $825-828$.

Pfau, C.J., Saron, M.F., Pevear, D.C., 1985. Lack of correlation between cytotoxic T lymphocytes and lethal murine lymphocytic choriomeningitis. J. Immunol. 135, 597-602.

Piechnik, S.K., Summers, P.E., Jezzard, P., Byrne, J.V., 2008. Magnetic resonance measurement of blood and CSF flow rates with phase contrast--normal values, repeatability and $\mathrm{CO}_{2}$ reactivity. Acta Neurochir. Suppl. 102, 263-270.

Pietrobon, D., Moskowitz, M.A., 2013. Pathophysiology of migraine. Annu. Rev. Physiol. 75, 365391.

Pietrobon, D., Moskowitz, M.A., 2014. Chaos and commotion in the wake of cortical spreading depression and spreading depolarizations. Nat. Rev. Neurosci. 15, 379-393.

Planaguma, J., Leypoldt, F., Mannara, F., Gutierrez-Cuesta, J., Martin-Garcia, E., Aguilar, E., Titulaer, M.J., Petit-Pedrol, M., Jain, A., Balice-Gordon, R., Lakadamyali, M., Graus, F., Maldonado, R., Dalmau, J., 2015. Human N-methyl D-aspartate receptor antibodies alter memory and behaviour in mice. Brain 138, 94-109.

Polfliet, M.M., Zwijnenburg, P.J., van Furth, A.M., van der Poll, T., Dopp, E.A., Renardel de Lavalette, C., van Kesteren-Hendrikx, E.M., van Rooijen, N., Dijkstra, C.D., van den Berg, T.K., 2001. Meningeal and perivascular macrophages of the central nervous system play a protective role during bacterial meningitis. J. Immunol. 167, 4644-4650.

Pollay, M., 2010. The function and structure of the cerebrospinal fluid outflow system. Cerebrospinal Fluid Res. 7, 9.

Pollay, M., Curl, F., 1967. Secretion of cerebrospinal fluid by the ventricular ependyma of the rabbit. Am. J. Physiol. 213, 1031-1038.

Preston, J.E., 2001. Ageing choroid plexus-cerebrospinal fluid system. Microsc. Res. Tech. 52, 3137.

Preston, S.D., Steart, P.V., Wilkinson, A., Nicoll, J.A., Weller, R.O., 2003. Capillary and arterial cerebral amyloid angiopathy in Alzheimer's disease: defining the perivascular route for the elimination of amyloid beta from the human brain. Neuropathol. Appl. Neurobiol. 29, 106-117. 
Price, R.W., Brew, B., Sidtis, J., Rosenblum, M., Scheck, A.C., Cleary, P., 1988. The brain in AIDS: central nervous system HIV-1 infection and AIDS dementia complex. Science 239, 586-592.

Price, T.J., Flores, C.M., 2007. Critical evaluation of the colocalization between calcitonin generelated peptide, substance $P$, transient receptor potential vanilloid subfamily type 1 immunoreactivities, and isolectin B4 binding in primary afferent neurons of the rat and mouse. J. Pain 8, 263-272.

Prineas, J.W., 1979. Multiple sclerosis: presence of lymphatic capillaries and lymphoid tissue in the brain and spinal cord. Science 203, 1123-1125.

Prodinger, C., Bunse, J., Kruger, M., Schiefenhovel, F., Brandt, C., Laman, J.D., Greter, M., Immig, K., Heppner, F., Becher, B., Bechmann, I., 2011. CD11c-expressing cells reside in the juxtavascular parenchyma and extend processes into the glia limitans of the mouse nervous system. Acta Neuropathol. 121, 445-458.

Puy, V., Zmudka-Attier, J., Capel, C., Bouzerar, R., Serot, J.M., Bourgeois, A.M., Ausseil, J., Baledent, O., 2016. Interactions between flow oscillations and biochemical parameters in the cerebrospinal fluid. Front. Aging Neurosci. 8, 154.

Quincke, H., 1872. Zur Physiologie der Cerebrospinalflussigkeit. Arch. Anat. Physiol. wiss. Med. $153-177$.

Quintana, E., Fernandez, A., Velasco, P., de Andres, B., Liste, I., Sancho, D., Gaspar, M.L., Cano, E., 2015. DNGR-1(+) dendritic cells are located in meningeal membrane and choroid plexus of the noninjured brain. Glia 63, 2231-2248.

Randolph, G., Merad, M., 2013. Reply to: "Can DCs be distinguished from macrophages by molecular signatures?". Nat. Immunol. 14, 189-190.

Rangroo Thrane, V., Thrane, A.S., Plog, B.A., Thiyagarajan, M., Iliff, J.J., Deane, R., Nagelhus, E.A., Nedergaard, M., 2013. Paravascular microcirculation facilitates rapid lipid transport and astrocyte signaling in the brain. Sci. Rep. 3, 2582.

Ransohoff, R.M., Engelhardt, B., 2012. The anatomical and cellular basis of immune surveillance in the central nervous system. Nat. Rev. Immunol. 12, 623-635.

Ransohoff, R.M., Hafler, D.A., Lucchinetti, C.F., 2015. Multiple sclerosis-a quiet revolution. Nat. Rev. Neurol. 11, 134-142.

Ransohoff, R.M., Kivisakk, P., Kidd, G., 2003. Three or more routes for leukocyte migration into the central nervous system. Nat. Rev. Immunol. 3, 569-581.

Ransom, B.R., Carlini, W.G., Yamate, C.L., 1987. Tip size of ion-exchanger based K+-selective microelectrodes. II. Effects on measurement of evoked $\left[\mathrm{K}^{+}\right]_{0}$ transients. Can. J. Physiol. Pharmacol. 65, 894-897.

Ray, B.S., Wolff, H.G., 1940. Experimental studies on headache. Pain sensitive structures of the head and their significance in headache. Arch. Surg. 41, 813-856.

Reboldi, A., Coisne, C., Baumjohann, D., Benvenuto, F., Bottinelli, D., Lira, S., Uccelli, A., Lanzavecchia, A., Engelhardt, B., Sallusto, F., 2009. C-C chemokine receptor 6-regulated entry of TH-17 cells into the CNS through the choroid plexus is required for the initiation of EAE. Nat. Immunol. 10, 514-523.

Reese, T.S., Karnovsky, M.J., 1967. Fine structural localization of a blood-brain barrier to exogenous peroxidase. J. Cell Biol. 34, 207-217.

Rennels, M.L., Gregory, T.F., Blaumanis, O.R., Fujimoto, K., Grady, P.A., 1985. Evidence for a 'paravascular' fluid circulation in the mammalian central nervous system, provided by the rapid distribution of tracer protein throughout the brain from the subarachnoid space. Brain Res. 326, 47-63.

Reuss, S., Riemann, R., Vollrath, L., 1992. Substance P- and calcitonin gene-related peptide-like immunoreactive neurons in the rat trigeminal ganglion--with special reference to meningeal and pineal innervation. Acta Histochem. 92, 104-109. 
Reuter, U., Bolay, H., Jansen-Olesen, I., Chiarugi, A., Sanchez del Rio, M., Letourneau, R., Theoharides, T.C., Waeber, C., Moskowitz, M.A., 2001. Delayed inflammation in rat meninges: implications for migraine pathophysiology. Brain 124, 2490-2502.

Reynier-Rebuffel, A.M., Callebert, J., Launay, J.M., Seylaz, J., Aubineau, P., 1997. NE inhibits cerebrovascular mast cell exocytosis induced by cholinergic and peptidergic agonists. Am. J. Physiol. 273, R845-850.

Ribatti, D., 2015. The crucial role of mast cells in blood-brain barrier alterations. Exp. Cell Res. 338, 119-125.

Ringstad, G., Emblem, K.E., Eide, P.K., 2016. Phase-contrast magnetic resonance imaging reveals net retrograde aqueductal flow in idiopathic normal pressure hydrocephalus. J. Neurosurg. 124, 1850-1857.

Rio-Hortega, P.d., 1919. Poder fagocitario y movilidad de la microglia. Bol. de la Soc. esp. de Biol. 9, 154-166.

Roberts, K.F., Elbert, D.L., Kasten, T.P., Patterson, B.W., Sigurdson, W.C., Connors, R.E., Ovod, V., Munsell, L.Y., Mawuenyega, K.G., Miller-Thomas, M.M., Moran, C.J., Cross, D.T., 3rd, Derdeyn, C.P., Bateman, R.J., 2014. Amyloid-beta efflux from the central nervous system into the plasma. Ann. Neurol. 76, 837-844.

Robin, C., 1859. Recherches sur quelques particularites de la structure des capillaires de l'encephale. J. Physiol. Homme Animaux 2, 537-548.

Rodriguez-Peralta, L.A., 1957. The role of the meningeal tissues in the hematoencephalic barrier. J. Comp. Neurol. 107, 455-473.

Rogers, L., 1931. The Foramen of Magendie. J. Anat. 65, 457-467.

Rosenberg, G.A., Kyner, W.T., Estrada, E., 1980. Bulk flow of brain interstitial fluid under normal and hyperosmolar conditions. Am. J. Physiol. 238, F42-49.

Rosenfeld, M.G., Mermod, J.J., Amara, S.G., Swanson, L.W., Sawchenko, P.E., Rivier, J., Vale, W.W., Evans, R.M., 1983. Production of a novel neuropeptide encoded by the calcitonin gene via tissue-specific RNA processing. Nature 304, 129-135.

Roth, T.L., Nayak, D., Atanasijevic, T., Koretsky, A.P., Latour, L.L., McGavern, D.B., 2014. Transcranial amelioration of inflammation and cell death after brain injury. Nature 505, 223-228.

Rotky, H., 1912. Untersuchungen über die Durchlässigkeit der Meningen für chemische Stoffe. Zeitschr. klin. Med. 75, 494 - 500.

Rozniecki, J.J., Dimitriadou, V., Lambracht-Hall, M., Pang, X., Theoharides, T.C., 1999. Morphological and functional demonstration of rat dura mater mast cell-neuron interactions in vitro and in vivo. Brain Res. 849, 1-15.

Rua, R., McGavern, D.B., 2015. Elucidation of monocyte/macrophage dynamics and function by intravital imaging. J. Leukoc. Biol. 98, 319-332.

Ruskell, G.L., 1988. The tentorial nerve in monkeys is a branch of the cavernous plexus. J. Anat. $157,67-77$.

Russo, A.F., 2015. CGRP as a neuropeptide in migraine: lessons from mice. Br. J. Clin. Pharmacol. 80, 403-414.

Russo, M.V., McGavern, D.B., 2015. Immune Surveillance of the CNS following Infection and Injury. Trends Immunol. 36, 637-650.

Saito, K., Liu-Chen, L.Y., Moskowitz, M.A., 1987. Substance P-like immunoreactivity in rat forebrain leptomeninges and cerebral vessels originates from the trigeminal but not sympathetic ganglia. Brain Res. 403, 66-71.

Sanchez-Valverde, M.A., Laredo, F.G., Lopez, F., Agut, A., Murciano, J., 1995. Method of recording cerebrospinal fluid pressure at three anatomic places in conscious and unrestrained adult wistar rats. Neurosci. Res. 24, 103-107.

Saria, A., Lundberg, J.M., Skofitsch, G., Lembeck, F., 1983. Vascular protein linkage in various tissue induced by substance $\mathrm{P}$, capsaicin, bradykinin, serotonin, histamine and by antigen challenge. Naunyn Schmiedebergs Arch. Pharmacol. 324, 212-218. 
Satpathy, A.T., Kc, W., Albring, J.C., Edelson, B.T., Kretzer, N.M., Bhattacharya, D., Murphy, T.L., Murphy, K.M., 2012. Zbtb46 expression distinguishes classical dendritic cells and their committed progenitors from other immune lineages. J. Exp. Med. 209, 1135-1152.

Sayed, B.A., Christy, A.L., Walker, M.E., Brown, M.A., 2010. Meningeal mast cells affect early T cell central nervous system infiltration and blood-brain barrier integrity through TNF: a role for neutrophil recruitment? J. Immunol. 184, 6891-6900.

Schachenmayr, W., Friede, R.L., 1978. The origin of subdural neomembranes. I. Fine structure of the dura-arachnoid interface in man. Am. J. Pathol. 92, 53-68.

Schley, D., Carare-Nnadi, R., Please, C.P., Perry, V.H., Weller, R.O., 2006. Mechanisms to explain the reverse perivascular transport of solutes out of the brain. J. Theor. Biol. 238, 962-974.

Schoonman, G.G., van der Grond, J., Kortmann, C., van der Geest, R.J., Terwindt, G.M., Ferrari, M.D., 2008. Migraine headache is not associated with cerebral or meningeal vasodilatation a 3 T magnetic resonance angiography study. Brain 131, 2192-2200.

Schroth, G., Klose, U., 1992a. Cerebrospinal fluid flow. I. Physiology of cardiac-related pulsation. Neuroradiology 35, 1-9.

Schroth, G., Klose, U., 1992b. Cerebrospinal fluid flow. II. Physiology of respiration-related pulsations. Neuroradiology 35, 10-15.

Schueler, M., Messlinger, K., Dux, M., Neuhuber, W.L., De Col, R., 2013. Extracranial projections of meningeal afferents and their impact on meningeal nociception and headache. Pain 154,1622-1631.

Schueler, M., Neuhuber, W.L., De Col, R., Messlinger, K., 2014. Innervation of rat and human dura mater and pericranial tissues in the parieto-temporal region by meningeal afferents. Headache 54, 996-1009.

Schwalbe, G., 1869. Die Arachnoidalraum ein Lymphraum und sein Zusammenhang mit den Perichorioidalraum. Z. med. Wiss. 7, 465 - 467.

Schwenger, N., Dux, M., de Col, R., Carr, R., Messlinger, K., 2007. Interaction of calcitonin generelated peptide, nitric oxide and histamine release in neurogenic blood flow and afferent activation in the rat cranial dura mater. Cephalalgia 27, 481-491.

Scollato, A., Tenenbaum, R., Bahl, G., Celerini, M., Salani, B., Di Lorenzo, N., 2008. Changes in aqueductal CSF stroke volume and progression of symptoms in patients with unshunted idiopathic normal pressure hydrocephalus. AJNR Am. J. Neuroradiol. 29, 192-197.

Seo, Y., Takamata, A., Ogino, T., Morita, H., Nakamura, S., Murakami, M., 2002. Water permeability of capillaries in the subfornical organ of rats determined by Gd-DTPA(2-) enhanced 1H magnetic resonance imaging. J. Physiol. 545, 217-228.

Shaw, T., Stewart-Hutchinson, P.J., Coles, J.A., van Rooijen, N., Zindy, E., $\quad$ Rodriquez, A., Brewer, J.M., Couper, K.N., Dustin, M.L., 2015. Perivascular arrest of CD8+ T cells is a signature of experimental cerebral malaria. PLoS Pathog. 11(11), e1005210.

doi:10.1371/journal.ppat.1005210

Shevel, E., 2012. The dura and migraine. Future Neurol. 7, 573-580.

Shibata, M., Yamada, S., Kumar, S.R., Calero, M., Bading, J., Frangione, B., Holtzman, D.M., Miller, C.A., Strickland, D.K., Ghiso, J., Zlokovic, B.V., 2000. Clearance of Alzheimer's amyloid-ss (1-40) peptide from brain by LDL receptor-related protein-1 at the blood-brain barrier. J. Clin. Invest. 106, 1489-1499.

Shih, A.Y., Driscoll, J.D., Drew, P.J., Nishimura, N., Schaffer, C.B., Kleinfeld, D., 2012. Twophoton microscopy as a tool to study blood flow and neurovascular coupling in the rodent brain. J. Cereb. Blood. Flow Metab. 32, 1277-1309.

Shimizu, T., Toriumi, H., Sato, H., Shibata, M., Nagata, E., Gotoh, K., Suzuki, N., 2007. Distribution and origin of TRPV1 receptor-containing nerve fibers in the dura mater of rat. Brain Res. 1173, 84-91.

Shukla, V., Hayman, L.A., Ly, C., Fuller, G., Taber, K.H., 2002. Adult cranial dura I: intrinsic vessels. J. Comput. Assist. Tomogr. 26, 1069-1074. 
Siegenthaler, J.A., Ashique, A.M., Zarbalis, K., Patterson, K.P., Hecht, J.H., Kane, M.A., Folias, A.E., Choe, Y., May, S.R., Kume, T., Napoli, J.L., Peterson, A.S., Pleasure, S.J., 2009. Retinoic acid from the meninges regulates cortical neuron generation. Cell 139, 597-609.

Siegenthaler, J.A., Pleasure, S.J., 2011. We have got you 'covered': how the meninges control brain development. Curr. Opin. Genet. Dev. 21, 249-255.

Silver, R., Curley, J.P., 2013. Mast cells on the mind: new insights and opportunities. Trends Neurosci. 36, 513-521.

Silverberg, G.D., Mayo, M., Saul, T., Rubenstein, E., McGuire, D., 2003. Alzheimer's disease, normal-pressure hydrocephalus, and senescent changes in CSF circulatory physiology: a hypothesis. Lancet Neurol. 2, 506-511.

Sixt, M.L., Messlinger, K., Fischer, M.J., 2009. Calcitonin gene-related peptide receptor antagonist olcegepant acts in the spinal trigeminal nucleus. Brain 132, 3134-3141.

Sminia, T., Dijkstra, C.D., 1986. The origin of osteoclasts: an immunohistochemical study on macrophages and osteoclasts in embryonic rat bone. Calcif. Tissue Int. 39, 263-266.

Sokolov, A.Y., Lyubashina, O.A., Amelin, A.V., Panteleev, S.S., 2013. The role of noradrenalin in the pathogenesis of primary headaches. Neurochem. J, 7, 256-265.

Spatz, H., 1934. Die Bedeutung der vitalen Farbung für die Lehre vom Stoffaustausch zwischen dem Zentralnervensystem und dem übrigen Körper. Arch. Psychiat. 101, 267-358.

Spector, R., 2009. Nutrient transport systems in brain: 40 years of progress. J. Neurochem. 111, 315-320.

Spector, R., Johanson, C.E., 2014. The nexus of vitamin homeostasis and DNA synthesis and modification in mammalian brain. Mol. Brain. 7, 3.

Spector, R., Keep, R.F., Snodgrass, S. R., Smith, Q.R., Johanson, C.E., 2015a. A balanced view of choroid plexus structure and function: Focus on adult humans. Exp. Neurol. 267, 78-86.

Spector, R., Snodgrass, S. R., Johanson, C.E., 2015b. A balanced view of the cerebrospinal fluid composition and functions: Focus on adult humans. Exp. Neurol. 273, 57-68.

Spina, A., 1899. Experimentelle Untersuchungen ueber die Bildung des Liquor cerebrospinalis. Archiv. für die gesammte Physiol. 76, 204-218.

Spitzer, N.C., 2015. Neurotransmitter Switching? No Surprise. Neuron 86, 1131-1144.

Starcevic, V.P., Morrow, B.A., Farner, L.A., Keil, L.C., Severs, W.B., 1988. Long-term recording of cerebrospinal fluid pressure in freely behaving rats. Brain Res. 462, 112-117.

Stefanovic, B., Hutchinson, E., Yakovleva, V., Schram, V., Russell, J.T., Belluscio, L., Koretsky, A.P., Silva, A.C., 2008. Functional reactivity of cerebral capillaries. J. Cereb. Blood Flow Metab. 28, 961-972.

Steiger, H.J., Tew, J.M., Jr., Keller, J.T., 1982. The sensory representation of the dura mater in the trigeminal ganglion of the cat. Neurosci. Lett. 31, 231-236.

Stern, L., 1923a. La barrière hémato-encéphalique dans les conditions normales et dans les conditions pathologiques Arch. Suisses Neurol. Psychiatr. 13, 604-616.

Stern, L., 1923b. La barriere hémato-encéphalique en physiologie et en clinique. Schweiz. Med. Wschr. 4:792.

Strandgaard, S., Sigurdsson, S.T., 2008. Last Word on Point:Counterpoint: Sympathetic nervous activity does/does not influence cerebral blood flow. J. Appl. Physiol. 105, 1375.

Strassman, A.M., Levy, D., 2006. Response properties of dural nociceptors in relation to headache. J. Neurophysiol. 95, 1298-1306.

Strassman, A.M., Raymond, S.A., Burstein, R., 1996. Sensitization of meningeal sensory neurons and the origin of headaches. Nature 384, 560-564.

Strassman, A.M., Weissner, W., Williams, M., Ali, S., Levy, D., 2004. Axon diameters and intradural trajectories of the dural innervation in the rat. J. Comp. Neurol. 473, 364-376.

Suzuki, N., Hardebo, J.E., Kahrstrom, J., Owman, C., 1990a. Effect on cortical blood flow of electrical stimulation of trigeminal cerebrovascular nerve fibres in the rat. Acta Physiol. Scand. 138, 307-316. 
Suzuki, N., Hardebo, J.E., Kahrstrom, J., Owman, C., 1990b. Selective electrical stimulation of postganglionic cerebrovascular parasympathetic nerve fibers originating from the sphenopalatine ganglion enhances cortical blood flow in the rat. J. Cereb. Blood Flow Metab. 10, 383-391.

Suzuki, N., Hardebo, J.E., Owman, C., 1988. Origins and pathways of cerebrovascular vasoactive intestinal polypeptide-positive nerves in rat. J. Cereb. Blood Flow Metab. 8, 697-712.

Svensjo, E., Grega, G.J., 1986. Evidence for endothelial cell-mediated regulation of macromolecular permeability by postcapillary venules. Fed. Proc. 45, 89-95.

Svoboda, K., Denk, W., Kleinfeld, D., Tank, D.W., 1997. In vivo dendritic calcium dynamics in neocortical pyramidal neurons. Nature 385, 161-165.

Syed, A.U., Koide, M., Braas, K.M., May, V., Wellman, G.C., 2012. Pituitary adenylate cyclaseactivating polypeptide (PACAP) potently dilates middle meningeal arteries: implications for migraine. J. Mol. Neurosci. 48, 574-583.

Syková, E., Nicholson, C., 2008. Diffusion in brain extracellular space. Physiol. Rev. 88, 12771340.

Takeshita, Y., Ransohoff, R.M., 2012. Inflammatory cell trafficking across the blood-brain barrier: chemokine regulation and in vitro models. Immunol. Rev. 248, 228-239.

Tarasoff-Conway, J.M., Carare, R.O., Osorio, R.S., Glodzik, L., Butler, T., Fieremans, E., Axel, L., Rusinek, H., Nicholson, C., Zlokovic, B.V., Frangione, B., Blennow, K., Menard, J., Zetterberg, H., Wisniewski, T., de Leon, M.J., 2015. Clearance systems in the brain implications for Alzheimer disease. Nat. Rev. Neurol. 11, 457-470.

ter Laan, M., van Dijk, J.M., Elting, J.W., Staal, M.J., Absalom, A.R., 2013. Sympathetic regulation of cerebral blood flow in humans: a review. Br. J. Anaesth. 111, 361-367.

Terron, J.A., Martinez-Garcia, E., 2007. 5-HT7 receptor-mediated dilatation in the middle meningeal artery of anesthetized rats. Eur. J. Pharmacol. 560, 56-60.

Theele, D.P., Streit, W.J., 1993. A chronicle of microglial ontogeny. Glia 7, 5-8.

Theer, P., Denk, W., 2006. On the fundamental imaging-depth limit in two-photon microscopy. J. Opt. Soc. Am. A Opt. Image Sci. Vis. 23, 3139-3149.

Theoharides, T.C., Donelan, J., Kandere-Grzybowska, K., Konstantinidou, A., 2005. The role of mast cells in migraine pathophysiology. Brain Res. Brain Res Rev. 49, 65-76.

Thorne, R.G., Nicholson, C., 2006. In vivo diffusion analysis with quantum dots and dextrans predicts the width of brain extracellular space. Proc. Natl Acad. Sci. U S A 103, 5567-5572.

Thrane, A.S., Takano, T., Rangroo Thrane, V., Wang, F., Peng, W., Ottersen, O.P., Nedergaard, M., Nagelhus, E.A., 2013. In vivo NADH fluorescence imaging indicates effect of aquaporin-4 deletion on oxygen microdistribution in cortical spreading depression. J. Cereb. Blood Flow Metab. 33, 996-999.

Toriumi, H., Shimizu, T., Shibata, M., Unekawa, M., Tomita, Y., Tomita, M., Suzuki, N., 2011. Developmental and circulatory profile of the diploic veins. Microvasc. Res. 81, 97-102.

Tringali, G., Greco, M.C., Capuano, A., Guerriero, G., Curro, D., Navarra, P., 2012. Flupirtine inhibits calcitonin-gene related peptide release from rat brainstem in vitro. Neurosci. Lett. 506:332-335.

Trolard, D., 1892. Les Granulations de Pacchioni. Les Lacunes Veineuses de la Dure-mère. J. Anat. Physiol. 28, 28-57.

Tsai, S.H., Tew, J.M., McLean, J.H., Shipley, M.T., 1988. Cerebral arterial innervation by nerve fibers containing calcitonin gene-related peptide (CGRP): I. Distribution and origin of CGRP perivascular innervation in the rat. J. Comp. Neurol. 271, 435-444.

Tseng, H.K., Liu, C.P., Price, M.S., Jong, A.Y., Chang, J.C., Toffaletti, D.L., Betancourt-Quiroz, M., Frazzitta, A.E., Cho, W.L., Perfect, J.R., 2012. Identification of genes from the fungal pathogen Cryptococcus neoformans related to transmigration into the central nervous system. PLoS One 7, e45083. 
Tsutsumi, S., Nakamura, M., Tabuchi, T., Yasumoto, Y., Ito, M., 2013. Calvarial diploic venous channels: an anatomic study using high-resolution magnetic resonance imaging. Surg. Radiol. Anat. 35, 935-941.

Uddman, R., Edvinsson, L., Owman, C., Sundler, F., 1981. Perivascular substance P: occurrence and distribution in mammalian pial vessels. J. Cereb. Blood Flow Metab. 1, 227-232.

Uddman, R., Goadsby, P.J., Jansen, I., Edvinsson, L., 1993. PACAP, a VIP-like peptide: immunohistochemical localization and effect upon cat pial arteries and cerebral blood flow. J. Cereb. Blood Flow Metab. 13, 291-297.

Uddman, R., Hara, H., Edvinsson, L., 1989. Neuronal pathways to the rat middle meningeal artery revealed by retrograde tracing and immunocytochemistry. J. Auton. Nerv. Syst. 26, 69-75.

van de Beek, D., Brouwer, M.C., Thwaites, G.E., Tunkel, A.R., 2012. Advances in treatment of bacterial meningitis. Lancet 380, 1693-1702.

Van Dorpe, J., Smeijers, L., Dewachter, I., Nuyens, D., Spittaels, K., Van Den Haute, C., Mercken, M., Moechars, D., Laenen, I., Kuiperi, C., Bruynseels, K., Tesseur, I., Loos, R., Vanderstichele, H., Checler, F., Sciot, R., Van Leuven, F., 2000. Prominent cerebral amyloid angiopathy in transgenic mice overexpressing the london mutant of human APP in neurons. Am. J. Pathol. 157, 1283-1298.

Varvel, N.H., Grathwohl, S.A., Baumann, F., Liebig, C., Bosch, A., Brawek, B., Thal, D.R., Charo, I.F., Heppner, F.L., Aguzzi, A., Garaschuk, O., Ransohoff, R.M., Jucker, M., 2012. Microglial repopulation model reveals a robust homeostatic process for replacing CNS myeloid cells. Proc. Natl Acad. Sci. U S A 109, 18150-18155.

Vasilache, A.M., Qian, H., Blomqvist, A., 2015. Immune challenge by intraperitoneal administration of lipopolysaccharide directs gene expression in distinct blood-brain barrier cells toward enhanced prostaglandin E(2) signaling. Brain Behav. Immun. 48, 31-41.

Veiga-Fernandes, H., Coles, M.C., Foster, K.E., Patel, A., Williams, A., Natarajan, D., Barlow, A., Pachnis, V., Kioussis, D., 2007. Tyrosine kinase receptor RET is a key regulator of Peyer's patch organogenesis. Nature 446, 547-551.

Verant, P., Serduc, R., Van Der Sanden, B., Remy, C., Vial, J.C., 2007. A direct method for measuring mouse capillary cortical blood volume using multiphoton laser scanning microscopy. J. Cereb. Blood Flow Metab. 27, 1072-1081.

Vergnano, S., Kadambari, S., Whalley, K., Menson, E.N., Martinez-Alier, N., Cooper, M., Sanchez, E., Heath, P.T., Lyall, H., 2015. Characteristics and outcomes of human parechovirus infection in infants (2008-2012). Eur. J. Pediatr. 174, 919-924.

Verlooy, J., Selosse, P., Van Reempts, J., Haseldonckx, M., Borgers, M., 1990. Fiberoptic intracranial pressure monitoring in rats. J. Neurosci. Methods 31, 1-6.

Vieussens, R. (1684) Neurographia universalis. Lyon (Lugduni).

Vintersten, K., Monetti, C., Gertsenstein, M., Zhang, P., Laszlo, L., Biechele, S., Nagy, A., 2004. Mouse in red: red fluorescent protein expression in mouse ES cells, embryos, and adult animals. Genesis 40, 241-246.

Virchow, R., 1851. Über die Erweiterung kleinerer Gefässe. Arch. Pathol. Anat. Physiol. (Virchows Archiv) 3, 427-462.

von Duering, M., Andres, K.H. 1991. Sensory nerve fiber terminals in the arachnoid granulations of non-human primates. Neurosci. Lett. 127, 121-124.

von Duering, M., Bauersachs, M., Bohmer, B., Veh, R.W., Andres, K.H., 1990. Neuropeptide Yand substance P-like immunoreactive nerve fibers in the rat dura mater encephali. Anat. Embryol. (Berl.) 182, 363-373.

von Recklinghausen, F.D. (1862) Die Lymphgefässe und ihre Beziehung zum Bindegewebe. Berlin: August Hirschwald.

Waggener, J.D., Beggs, J., 1967. The membranous coverings of neural tissues: an electron microscopy study. J. Neuropathol. Exp. Neurol. 26, 412-426. 
Wagner, H.J., Pilgrim, C., Brandl, J., 1974. Penetration and removal of horseradish peroxidase injected into the cerebrospinal fluid: role of cerebral perivascular spaces, endothelium and microglia. Acta Neuropathol. 27, 299-315.

Walker-Caulfield, M.E., Hatfield, J.K., Brown, M.A., 2015. Dynamic changes in meningeal inflammation correspond to clinical exacerbations in a murine model of relapsing-remitting multiple sclerosis. J. Neuroimmunol. 27, 112-122.

Wallace, G.B., Brodie, B.B., 1940. On the source of the cerebrospinal fluid. The distribution of bromide and iodide throughout the central nervous system. J. Pharmacol. Exp. Ther. 70, 418-427.

Walter, F.K., 1930. Die "Blut-Hirn-Schranke,". Z. f. d. ges. Neurol. u. Psychiat. 128, 580 - 586.

Wang, P., Olbricht, W.L., 2011. Fluid mechanics in the perivascular space. J. Theor. Biol. 274, 5257

Wang, X., Fang, Y., Liang, J., Yan, M., Hu, R., Pan, X., 2014. 5-HT7 receptors are involved in neurogenic dural vasodilatation in an experimental model of migraine. J. Mol. Neurosci. 54, 164-170.

Wårdell, K., Naver, H.K., Nilsson, G.E., Wallin, B.G., 1993. The cutaneous vascular axon reflex in humans characterized by laser Doppler perfusion imaging. J. Physiol. 460, 185-199.

Weed, L.H., 1914a. Studies on cerebro-spinal fluid. No.II. The theories of drainage of cerebrospinal fluid with an analysis of the methods of investigation. J. Med. Res. 31, 21 - 49.

Weed, L.H., 1914b. Studies on Cerebro-Spinal Fluid. No. III : The pathways of escape from the subarachnoid spaces with particular reference to the arachnoid villi. J. Med. Res. 31, 51-91.

Weed, L.H., 1914c. Studies on Cerebro-Spinal Fluid. No. IV : The dual source of cerebro-spinal fluid. J. Med. Res. 31, 93-118 111.

Weed, L.H., 1923. The absorption of cerebrospinal fluid into the venous system. Am. J. Anat. 31, $191-221$.

Weed, L.H., 1938. Meninges and cerebrospinal fluid. J. Anat. 72, 181-215.

Wei, X., Melemedjian, O.K., Ahn, D.D., Weinstein, N., Dussor, G., 2014. Dural fibroblasts play a potential role in headache pathophysiology. Pain 155, 1238-1244.

Welch, K., Friedman, V., 1960. The cerebrospinal fluid valves. Brain 83, 454-469.

Weller, R.O., 2005. Microscopic morphology and histology of the human meninges. Morphologie $89,22-34$.

Weller, R.O., Djuanda, E., Yow, H.Y., Carare, R.O., 2009. Lymphatic drainage of the brain and the pathophysiology of neurological disease. Acta Neuropathol. 117, 1-14.

Weller, R.O., Massey, A., Newman, T.A., Hutchings, M., Kuo, Y.M., Roher, A.E., 1998. Cerebral amyloid angiopathy: amyloid beta accumulates in putative interstitial fluid drainage pathways in Alzheimer's disease. Am. J. Pathol. 153, 725-733.

Weller, R.O., Subash, M., Preston, S.D., Mazanti, I., Carare, R.O., 2008. Perivascular drainage of amyloid-beta peptides from the brain and its failure in cerebral amyloid angiopathy and Alzheimer's disease. Brain Pathol. 18, 253-266.

Wen, H., Nagelhus, E.A., Amiry-Moghaddam, M., Agre, P., Ottersen, O.P., Nielsen, S., 1999. Ontogeny of water transport in rat brain: postnatal expression of the aquaporin-4 water channel. Eur. J. Neurosci. 11, 935-945.

Widner, H., Jonsson, B.A., Hallstadius, L., Wingardh, K., Strand, S.E., Johansson, B.B., 1987. Scintigraphic method to quantify the passage from brain parenchyma to the deep cervical lymph nodes in rats. Eur. J. Nucl. Med. 13, 456-461.

Williamson, D.J., Hargreaves, R.J., Hill, R.G., Shepheard, S.L., 1997a. Intravital microscope studies on the effects of neurokinin agonists and calcitonin gene-related peptide on dural vessel diameter in the anaesthetized rat. Cephalalgia 17, 518-524.

Williamson, D.J., Hargreaves, R.J., Hill, R.G., Shepheard, S.L., 1997b. Sumatriptan inhibits neurogenic vasodilation of dural blood vessels in the anaesthetized rat - intravital microscope studies. Cephalalgia 17, 525-531.

Willis, T. (1664) Cerebri Anatome cui accessit nervorum descripto et usus. London: D. Paul. 
Wisniewski, H.M., Wegiel, J., 1994. Beta-amyloid formation by myocytes of leptomeningeal vessels. Acta Neuropathol. 87, 233-241.

Wisniewski, H.M., Wegiel, J., Wang, K.C., Lach, B., 1992. Ultrastructural studies of the cells forming amyloid in the cortical vessel wall in Alzheimer's disease. Acta Neuropathol. 84, 117-127.

Wolf, S.A., Steiner, B., Akpinarli, A., Kammertoens, T., Nassenstein, C., Braun, A., Blankenstein, T., Kempermann, G., 2009. CD4-positive T lymphocytes provide a neuroimmunological link in the control of adult hippocampal neurogenesis. J. Immunol. 182, 3979-3984.

Wolff, H.G., 1930. Cerebral circulation XII. The effect on pial vessels of variation in the oxygen and carbon dioxide content of the blood. Arch. Neurol. Psychiat. 23, 1097-1120.

Woods, S.J., Saron, M.F., Pfau, C.J., 1987. Meningeal macrophages reflect lymphocytic choriomeningitis virus pathogenic phenotypes. Scand. J. Immunol. 26, 97-103.

Woollam, D.H., Millen, J.W., 1953. An anatomical approach to poliomyelitis. Lancet 261, 364367.

Woollam, D.H., Millen, J.W., 1955. The perivascular spaces of the mammalian central nervous system and their relation to the perineuronal and subarachnoid spaces. J. Anat. 89, 193-200.

Xie, L., Kang, H., Xu, Q., Chen, M.J., Liao, Y., Thiyagarajan, M., O'Donnell, J., Christensen, D.J., Nicholson, C., Iliff, J.J., Takano, T., Deane, R., Nedergaard, M., 2013. Sleep drives metabolite clearance from the adult brain. Science 342, 373-377.

Xu, H.L., Mao, L., Ye, S., Paisansathan, C., Vetri, F., Pelligrino, D.A., 2008. Astrocytes are a key conduit for upstream signaling of vasodilation during cerebral cortical neuronal activation in vivo. Am. J. Physiol. Heart. Circ. Physiol. 294, H622-632.

Xu, H.T., Pan, F., Yang, G., Gan, W.B., 2007. Choice of cranial window type for in vivo imaging affects dendritic spine turnover in the cortex. Nat. Neurosci. 10, 549-551.

Yamaguchi, H., Yamazaki, T., Lemere, C.A., Frosch, M.P., Selkoe, D.J., 1992. Beta amyloid is focally deposited within the outer basement membrane in the amyloid angiopathy of Alzheimer's disease. An immunoelectron microscopic study. Am. J. Pathol. 141, 249-259.

Yamate, J., Ishimine, S., Izawa, T., Kumagai, D., Kuwamura, M., 2009. Macrophage populations and expressions of regulatory proinflammatory factors in the rat meninx under lipopolysaccharide treatment in vivo and in vitro. Histol. Histopathol. 24, 13-24.

Yamashima, T., Friede, R.L., 1984. Light and electron microscopic studies on the subdural space, the subarachnoid space and the arachnoid membrane. Neurol. Med. Chir. (Tokyo) 24, 737-746.

Yamazumi, H., 1989. [Infiltration of India ink from subarachnoid space to nasal mucosa along olfactory nerves in rabbits]. Nihon Jibiinkoka Gakkai Kaiho 92, 608-616.

Yan, J., Wei, X., Bischoff, C., Edelmayer, R.M., Dussor, G., 2013. pH-evoked dural afferent signaling is mediated by ASIC3 and is sensitized by mast cell mediators. Headache 53, 1250-1261.

Yang, G., Pan, F., Parkhurst, C.N., Grutzendler, J., Gan, W.B., 2010. Thinned-skull cranial window technique for long-term imaging of the cortex in live mice. Nat. Protoc. 5, 201-208.

Yasuda, K., Cline, C., Vogel, P., Onciu, M., Fatima, S., Sorrentino, B.P., Thirumaran, R.K., Ekins, S., Urade, Y., Fujimori, K., Schuetz, E.G., 2013. Drug transporters on arachnoid barrier cells contribute to the blood-cerebrospinal fluid barrier. Drug Metab. Dispos. 41, 923-931.

Yildiz, S., Thyagaraj, S., Jin, N., Zhong, X., Heidari Pahlavian, S., Martin, B.A., Loth, F., Oshinski, J., Sabra, K.G., 2017. Quantifying the influence of respiration and cardiac pulsations on cerebrospinal fluid dynamics using real-time phase-contrast MRI. J. Magn. Reson. Imaging. doi: 10.1002/jmri.25591.

Yoon, S., Zuccarello, M., Rapoport, R.M., 2012. $\mathrm{pCO}_{2}$ and $\mathrm{pH}$ regulation of cerebral blood flow. Front. Physiol. 3, 365. 
Yoshida, K., Takahashi, H., Saijo, M., Ueguchi, T., Tanaka, H., Fujita, N., Murase, K., 2009. Phase-contrast MR studies of CSF flow rate in the cerebral aqueduct and cervical subarachnoid space with correlation-based segmentation. Magn. Reson. Med. Sci. 8, 91-100.

Zajicova, A., 1987. Ontogenetic development of the dura mater encephali et spinalis of the laboratory mouse Mus musculus v. alba. Folia Morphol. (Praha) 35, 46-52.

Zakharov, A., Papaiconomou, C., Koh, L., Djenic, J., Bozanovic-Sosic, R., Johnston, M., 2004. Integrating the roles of extracranial lymphatics and intracranial veins in cerebrospinal fluid absorption in sheep. Microvasc. Res. 67, 96-104.

Zenker, W., Kubik, S., 1996. Brain cooling in humans--anatomical considerations. Anat. Embryol. (Berl.) 193, 1-13.

Zhang, E.T., Richards, H.K., Kida, S., Weller, R.O., 1992. Directional and compartmentalised drainage of interstitial fluid and cerebrospinal fluid from the rat brain. Acta Neuropathol. 83, 233-239.

Zhang, X.C., Kainz, V., Jakubowski, M., Burstein, R., Strassman, A., Levy, D., 2009. Localization of COX-1 and COX-2 in the intracranial dura mater of the rat. Neurosci. Lett. 452, 33-36.

Zhao, J., Levy, D., 2014. The sensory innervation of the calvarial periosteum is nociceptive and contributes to headache-like behavior. Pain 155, 1392-1400.

Zhao, J., Levy, D., 2015. Modulation of intracranial meningeal nociceptor activity by cortical spreading depression: a reassessment. J. Neurophysiol. 113, 2778-2785.

Zhu, X., Hill, R.A., Nishiyama, A., 2008. NG2 cells generate oligodendrocytes and gray matter astrocytes in the spinal cord. Neuron Glia Biol. 4, 19-26.

Zwillinger, H., 1912. Die Lymphbahnen des oberen Nasalschnittes und deren Beziehungen zu den perimeningealen Lymphraümen. Arch. Laryngol. Rhinol. 26, 66-78. 